\title{
Discourse Representation in Context
}

\author{
Jan van Eijck and Hans Kamp
}

January 7, 2010

\section{Overview}

Discourse representation in context is the attempt to capture certain aspects of the interpretation of natural language texts that are beyond the mere truth conditions of the text. Prime examples are interpretation of indefinites and pronouns in context, and interpretation of tenses, in French and other languages.

One of the debates surrounding the advent of discourse representation theory (DRT, [37]) and file change semantics (FCS, [35]) had to do with the issue of representationalism. Should we assume the representation structures to say something about what goes on in the mind of the interpreter, or not. On this issue, the followers of the Montague tradition tend to have strongly antimentalist views. Semantics, in the Montagovian perspective, is not about what goes on in the mind, but about how language relates to reality.

Montague tried to settle the issue of representation languages ('logical form') once and for all by means of a careful demonstration that immediate interpretation of natural language fragments in appropriate models, without an intervening logical form, was possible. DRT and FSC, in their original presentations, re-introduced logical forms into the picture. The first attempts at rational reconstruction of DRT and FCS were geared at showing that the representation language (the boxes of DRT) could be eliminated again. This led to the development of compositional versions of DRT such as dynamic predicate logic (DPL), and dynamic versions of Montague grammar based on DPL. The snag was that these rational reconstructions were not quite faithful to the original enterprise. See [25] for a detailed account of the relationship between DRT and DPL, in the context of a historical study 
of dynamic logics in computer science and natural language analysis. An overview of DRT from the viewpoint of representationalism can be found in [40].

Anti-mentalism is less fashionable nowadays. Indeed, many researchers have come to view natural language analysis as a branch of cognitive science. But this new view creates new obligations. If one takes this connection with cognition seriously, one has to take on the burden of showing that the building of discourse representations, as it goes on in the theory, somehow corresponds with what goes on in the mind. It seems fair to say that this is a challenge that has yet to be met.

This paper is an update to our previous overview [24]. We will first introduce the purpose of the overall enterprise of discourse representation. Next, we focus on some technical issues, in order to clarify what goes on essentially when text is interpreted in context.

\section{Interpretation of Text in Context}

The fundamental idea behind the theory of the semantics of coherent multisentence discourse and text that is presented in this chapter-Discourse Representation Theory, or DRT for short - is that each new sentence $S$ of a discourse is interpreted in the context provided by the sentences preceding it. The result of this interpretation is that the context is updated with the contribution made by $S$; often an important part of this process is that anaphoric elements of $S$ are hooked up to elements that are present in the context. An implication of this conception of text interpretation is that one and the same structure serves simultaneously as content and as context - as content of the sentences that have been interpreted already and as context for the sentence that is to be interpreted next. This double duty imposes

special constraints on logical form, which are absent when, as in most older conceptions of semantics and pragmatics, the contents and contexts are kept separate.

The initial problem that motivated the present theory is the interpretation of nominal and temporal anaphora in discourse. The key idea in the way of thinking about the semantics of discourse in context exemplified in Heim [35] and Kamp [37] is that each new sentence or phrase is interpreted as an addition to, or 'update' of, the context in which it is used and that this update often involves connections between elements from the sentence or 
phrase with elements from the context.

In the approach of Kamp [37], which we will follow more closely here than the largely equivalent approach of Heim [35], this idea is implemented in the form of interpretation rules - each associated with a particular lexical item or syntactic construction. When applied to a given sentence $S$, these rules identify the semantic contributions which $S$ makes to the context $C$ in which $S$ is used and add these to $C$. In this way $C$ is transformed into a new context, which carries the information contributed by $S$ as well as the information that was part of the context already. The result can then serve as context for the interpretation of the sentence following $S$ (in the given discourse or text), which leads to yet another context, and so on until the entire discourse or text has been interpreted.

An important aspect of this kind of updating of contexts is the introduction of elements - so-called reference markers or discourse referents - that can serve as antecedents to anaphoric expressions in subsequent discourse. These reference markers play a key part in the the context structures posited by DRT, the so-called Discourse Representation Structures or DRSs.

With its emphasis on representing and interpreting discourse in context, discourse representation theory has been instrumental in the emergence of a dynamic perspective on natural language semantics, where the centre of the stage, occupied so long by the concept of truth with respect to appropriate models, has been replaced by context change conditions, with truth conditions defined in terms of those. Thus, under the influence of discourse representation theory, many traditional Montague grammarians have made the switch from static to dynamic semantics (see the Chapter on Dynamics in this Handbook). This shift has considerably enriched the enterprise of formal semantics, by bringing areas formerly belonging to informal pragmatics within its compass.

In the next section we will first look at some examples of DRSs and at the considerations which have led to their specific form. After that we will look more closely at the relationship between DRSs and the syntactic structure of sentences, discourses or texts from which they can be derived. This will lead us naturally to the much debated question whether the theory presented here is compositional. The compositionality issue will force us to look carefully at the operations by means of which DRSs can be put together from minimal building blocks. Next we will show, by developing a toy example, what a compositional discourse semantics for a fragment of natural language may 
look like. This is followed by sample treatments of quantification, tense and aspect. The chapter ends with some pointers to the literature on further extensions of the approach and to connections with related approaches.

\section{The Problem of Anaphoric Linking in Context}

The semantic relationship between personal pronouns and their antecedents was long perceived as being of two kinds: a pronoun either functions as an individual constant coreferential with its antecedent or it acts as a variable bound by its antecedent. However, in the examples (1)-(4) below, neither of these two possibilities seems to provide a correct account of how pronoun and antecedent are related.

(1) A man ${ }^{1}$ entered. $\mathrm{He}_{1}$ smiled.

(2) Every man who meets a nice woman ${ }^{1}$ smiles at her $_{1}$.

(3) If a $\operatorname{man}^{1}$ enters, he 1 smiles.

(4) Hob believes a witch ${ }^{1}$ blighted his mare. Nob believes she ${ }_{1}$ killed his sow.

In these examples we have used subscripts and superscripts to coindex anaphoric pronouns and their intended antecedents.

The first option - of pronoun and antecedent being coreferential - does not work for the simple reason that the antecedent does not refer (as there is no one particular thing that can be counted as the referent!); so a fortiori antecedent and pronoun cannot corefer (that is, refer to the same thing). The second option, the bound variable analysis, runs into problems because the pronoun seems to be outside the scope of its antecedent. For instance, in (1) the antecedent of the pronoun is an indefinite noun phrase occurring in the preceding sentence. In the approaches which see pronouns as either coreferring terms or bound variables, indefinite NPs are viewed as existential quantifiers whose scope does not extend beyond the sentence in which they occur. In such an approach there is no hope of the pronoun getting properly bound. Examples (2)-(4) present similar difficulties. Example (2) is arguably ambiguous in that a nice woman may be construed either as having wide or as having narrow scope with respect to every man. If a nice woman is construed as having narrow scope, i.e. as having its scope restricted to the 
relative clause, then the pronoun won't be bound; the phrase can bind the pronoun if it is given wide scope, as in that case its scope is the entire sentence, but this leads to an interpretation which, though perhaps marginally possible, is clearly not the preferred reading of (2). We find much the same problem with (3): in order that the indefinite $a$ man bind the pronoun he, it must be construed as having scope over the conditional as a whole, and not just over the if-clause; but again, this yields a reading that is marginal at best, while the preferred reading is not available.

Sentences with the patterns of (2) and (3) have reached the modern semantic literature through Geach [31], who traces them back to the Middle Ages and beyond. Geach's discussion revolves around examples with donkeys, so these sentences became known in the literature as donkey sentences. Also due to Geach are sentences like (4), which pose a binding problem across a sentential boundary, complicated by the fact that antecedent and anaphoric element occur in the scopes of different attitude predications, with distinct subjects.

Problems like the ones we encountered with (1)-(4) arise not just with pronouns. There are several other types of expressions with anaphoric uses that present essentially the same difficulties to the traditional ways of viewing the relationship between natural language and logic. First, there are other anaphoric noun phrases besides pronouns, viz. definite descriptions and demonstratives; and these also occur in the contexts where the problems we have just noted arise. Moreover, as was remarked already more than twenty years ago in Partee [52], there are striking similarities in the behaviour of anaphoric pronouns and tenses, and it turns out that the interpretation of tense involves the same sort of anaphoric dependencies which (1)-(4) exhibit. More precisely, the past tense is often to be understood as referring to some particular time in the past (rather than meaning 'sometime in the past') and more often than not this particular time is to be recovered from the context in which the given past tense sentence is used.

(5) John entered the room. He switched on the light.

(6) Whenever John entered the room, he switched on the light.

In (5) the switching time is understood as temporally related to the time at which John entered the room (presumably the time of switching was directly after the time of entering) and a full interpretation of (5) needs to make this explicit. A quantificational sentence such as (6) suggests the 
same relationship between switching times and entering times; and insofar as the tense of the main clause is to be interpreted as anaphoric to that of the whenever-clause, this anaphoric connection raises the same questions as those of (2) and (3).

\section{Basic Ideas of Discourse Representation}

The central concepts of DRT are best explained with reference to simple examples such as (1) in the previous section. The logical content of (1) appears to be that there was some man who entered and (then) smiled. That is, the content of (1) is what in standard predicate logic would be expressed by an existential quantification over material coming in part from the first and in another part from the second sentence of (1), roughly as in (7).

$$
\exists x(\operatorname{man}(x) \wedge \text { entered }(x) \wedge \text { smiled }(x))
$$

As observed in the last section, according to DRT the interpretation of (1) results from a process in which an interpretation is obtained for the first sentence, which then serves as context for the interpretation of the second sentence. The interpretation of the second sentence transforms this context into a new context structure, the content of which is essentially that of (7).

The problem with (1) is that the first sentence has an existential interpretation and thus must in some way involve an existential quantifier, and that the contribution which the second sentence makes to the interpretation of (1) must be within the scope of that quantifier. Given the basic tenets of DRT, this means that (i) the first sentence of (1) must get assigned a representation, i.e. a DRS, $K_{1}$ which captures the existential interpretation of that sentence; and (ii) this DRS $K_{1}$ must be capable of acting as context for the interpretation of the second sentence in such a way that this second interpretation process transforms it into a DRS $K_{2}$ representing the truth conditions identified by (7). (i) entails that the reference marker introduced by the indefinite NP a man - let it be $x$-must get an existential interpretation within $K_{1}$; and (ii) entails that it is nevertheless available subsequently as antecedent for the pronoun he. Finally, after $x$ has been so exploited in the interpretation of the second sentence, it must then receive once more an existential interpretation within the resulting DRS $K_{2}$. 
Heim [35] uses the metaphor of a filing cabinet for this process. The established representation structure $K_{1}$ is a set of file cards, and additions to the discourse effect a new structure $K_{2}$, which is the result of changing the file in the light of the new information. Here is how DRT deals with these desiderata. The DRS $K_{1}$ is as given in (8).

\begin{tabular}{|l|}
\hline $\mathrm{x}$ \\
\hline man $\mathrm{x}$ \\
entered $\mathrm{x}$ \\
\hline
\end{tabular}

This can also be rendered in canonical set-theoretical notation, as in (9).

$$
(\{\mathrm{x}\},\{\text { man } \mathrm{x}, \text { entered } \mathrm{x}\})
$$

Precisely how this DRS is derived from the syntactic structure of the first sentence of (1), and how DRS construction from sentences and texts works generally is discussed in Section 10. For now, suffice it to note that the reference marker $x$ gets introduced when the NP a man is interpreted and that this interpretation also yields the two conditions $\operatorname{man}(x)$ and entered $(x)$, expressing that any admissible value $a$ for $x$ must be a man and that this man was one who entered.

A DRS like (8) can be viewed as a kind of 'model' of the situation which the represented discourse describes. The modelled situation contains at least one individual $a$, corresponding to the reference marker $x$, which satisfies the two conditions contained in (8), i.e. $a$ is a man and $a$ is someone who entered.

When a DRS is used as context in the interpretation of some sentence $S$, its reference markers may serve as antecedents for anaphoric NPs occurring in $S$. In the case of our example we have the following. (8), serving as context for the second sentence of (1), makes $x$ available as antecedent for the pronoun he. That is, the interpretation of he links the reference marker it introduces, $y$ say, to the marker $x$ for the intended antecedent, something we express by means of the equational condition $y \doteq x$. In addition, the interpretation step yields, as in the case of the indefinite $a$ man, a condition expressing the clausal predication which involves he as argument. Through the application of this principle (8) gets expanded to the DRS (10), which represents the content of all of (1). 


\begin{tabular}{|l|}
\hline $\mathrm{x} y$ \\
\hline $\operatorname{man} \mathrm{x}$ \\
enter $\mathrm{x}$ \\
$\mathrm{y} \doteq \mathrm{x}$ \\
smiled $\mathrm{y}$ \\
\end{tabular}

DRS (10) models situations in which there is at least one individual that is a man, that entered and that smiled. It is easy to see that these are precisely the situations which satisfy the predicate formula (7). (This claim will be made formal by the model theory for DRSs, to be presented in Section 5.)

As illustrated by the above examples (8) and (10), a DRS generally consists of two parts, (i) a set of reference markers, the universe of the DRS, and (ii) a set of conditions, its condition set. There are some other general points which our example illustrates:

1. The reference markers in the universe of a DRS all get an existential interpretation;

2. All reference markers in the universe of a context DRS are available as anaphoric antecedents to pronouns and other anaphoric expressions that are interpreted within this context;

3. The interpretation of a sentence $S$ in the context provided by a DRS $K$ results in a new DRS $K^{\prime}$, which captures not only the content represented by $K$ but also the content of $S$, as interpreted with respect to $K$.

It should be clear that DRSs such as (8) and (10) can only represent information that has the logical form of an existentially quantified conjunction of atomic predications. But there is much information that is not of this form. This is so, in particular, for the information expressed by (3). So the DRS for (3) will have to make use of representational devices different from those that we have used up to this point.

The DRT conception of conditional information is this. The antecedent of a conditional describes a situation, and the conditional asserts that this situation must also satisfy the information specified in its consequent. When conditionals are seen from this perspective, it is not surprising that the 
interpretation of their consequents may use the interpretations of their antecedents as contexts much in the way the interpretation of a sentence $S$ may build upon the interpretation assigned to the sentences preceding it in the discourse to which it belongs; for the consequent extends the situation description provided by the antecedent in essentially the same way in which $S$ extends the situation described by its predecessors.

In the case of (3) this means that the DRS (8), which represents its antecedent (see the discussion of (1) above), can be exploited in the interpretation of the consequent, just as (8), as interpretation of the first sentence of (1), supported the interpretation of the second sentence of (1). To make this work out, we need a suitable representation for the consequent. This turns out to be (11).

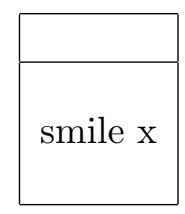

To obtain a representation of (3), (8) and (11) must be combined in a way which reveals the conditional connection between them. We represent this combination by a double arrow in between the two DRSs. The result $K \Rightarrow K^{\prime}$, where $K$ and $K^{\prime}$ are the two DRSs to be combined, is a DRS condition (a complex condition as opposed to the simple DRS conditions we have encountered so far). The DRS for a conditional sentence such as (3) will consist just of such a condition and nothing else.

Intuitively the meaning of a condition $K \Rightarrow K^{\prime}$ is that a situation satisfying $K$ also satisfies $K^{\prime}$. This is indeed the semantics we adopt for such conditions (for details see Section 5). Applying this to the case of (3) we get the representation (12).

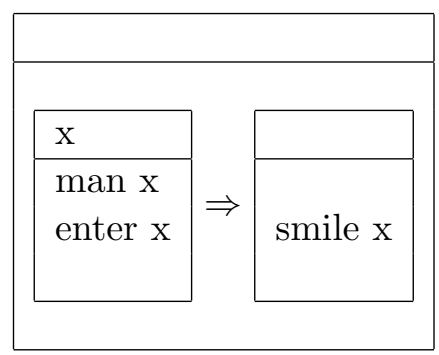

Conditions of the form $K \Rightarrow K^{\prime}$ illustrate an important feature of DRT: 
The logical role played by a reference marker depends on the DRS-universe to which it belongs. Markers belonging to the universe of the main DRS get an existential interpretation - this is, we saw, a consequence of the principle that a DRS is true if it is possible to find individuals corresponding to the reference markers in the DRS universe which satisfy its conditions. This principle, however, applies only to the reference markers in the main DRS universe. The logic of reference markers in subordinate universes, such as for instance $x$ in (12), is determined by the principles governing the complex DRS conditions to which they belong. Thus the semantics of conditions of the form $K \Rightarrow K^{\prime}$ implies that for all individuals corresponding to reference markers in the universe of $K$ which satisfy the conditions of $K$ it is the case that $K^{\prime}$ is satisfiable as well. Thus the $\Rightarrow$-condition of (12) has the meaning that for every individual corresponding to the marker $x$ - that is, for every man that enters - the right hand side DRS of (12) is satisfied, i.e. that individual smiles. Reference markers in the left hand side universe of an $\Rightarrow$-condition thus get a universal, not an existential interpretation.

It is worth noting explicitly the ingredients to this solution of the semantic dilemma posed by conditionals like (3). Crucial to the solution are:

1. the combination of the principles of DRS construction, which assign to conditional sentences such as (3) representations such as (12), and

2. the semantics for $\Rightarrow$-conditions that has just been described.

Like any other DRS, (12) is a pair consisting of a set of reference markers and a set of conditions. But in (12) the first of these sets is empty. In particular, the reference marker $x$ which does occur in (12) belongs not to the universe of the 'main' DRS of (12) but to that of a subordinate DRS, which itself is a constituent of some DRS condition occurring in (12). One important difference between reference markers in such subordinate positions and those belonging to the universe of the main DRS is that only the latter are accessible as antecedents for anaphoric pronouns in subsequent sentences. In general, in order that a reference marker can serve as antecedent to a subsequent pronoun, it must be accessible from the position that the pronoun occupies. Compare for instance the discourses (13) and (14).

(13) A man came in. He smiled. He was holding a flower in his right hand.

(14) If a man comes in, he smiles. ?He is holding a flower in his right hand. 
While in (13) the second he is as unproblematic as the first he, in (14) the second he is hard or impossible to process. This difference is reflected by the fact that in the DRS for the first two sentences of (13) the reference marker for a man belongs to the universe of the main DRS and so is accessible to the pronoun of the last sentence, whereas in (14) this is not so.

The rules for processing sentences in the context of a representation structure impose formal constraints on availability of discourse referents for anaphoric linking. The set of available markers consists of the markers of the current structure, plus the markers of structures that can be reached from the current one by a series of steps in the directions left, (i.e. from the consequent of a pair $K \Rightarrow K^{\prime}$ to the antecedent), and $u p$, (i.e. from a structure to an encompassing structure).

For universally quantified sentences such as (2) DRT offers an analysis that closely resembles its treatment of conditionals. According to this analysis a universally quantifying NP imposes a conditional connection between its own descriptive content and the information expressed by the predication in which it participates as argument phrase; and this connection is interpreted in the same way as the $\Rightarrow$-conditions that the theory uses to represent conditional sentences. In particular, (2) gets an analysis in which any individual satisfying the descriptive content man who meets a nice woman, i.e. any individual corresponding to the reference marker $x$ in the DRS (15), satisfies the DRS representing the main predication of (2). According to this way of looking at quantification, the descriptive content of the quantifying phrase can be taken as presupposed for purposes of interpreting the predication in which the phrase partakes, just as the antecedent of a conditional can be taken as given when interpreting its consequent. Thus, just as we saw for the consequent of the conditional (3), the construction of the DRS for the main predication of (2) may make use of information encoded in the 'descriptive content' DRS (15). The result is the DRS in (16).

\begin{tabular}{|l|}
\hline $\mathrm{x} y$ \\
\hline $\operatorname{man} \mathrm{x}$ \\
woman $\mathrm{y}$ \\
nice $\mathrm{y}$ \\
meet $(\mathrm{x}, \mathrm{y})$ \\
\end{tabular}




\begin{tabular}{|l|}
\hline $\mathrm{u}$ \\
$\mathrm{u} \doteq \mathrm{y}$ \\
smiles-at $(\mathrm{x}, \mathrm{u})$
\end{tabular}

To get a representation of (2), DRSs (15) and (16) have to be combined into a single DRS condition. It is clear that $\Rightarrow$ has the desired effect. The result is (17).

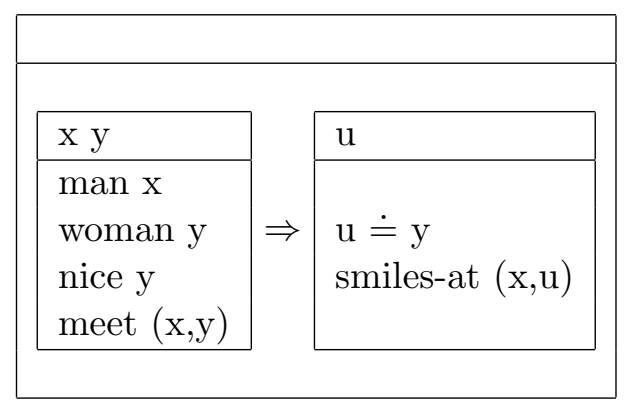

The constraints on marker accessibility are used to account for the awkwardness of anaphoric links as in (18).

(18) *If every $\operatorname{man}^{1}$ meets a nice woman ${ }^{2}$, he 1 smiles at her 2 .

The difference between pronominal anaphora and the variable binding we find in classical logic is also nicely illustrated by anaphora involving the word other. Consider e.g. (19).

(19) A man walked in. Another man followed him.

Here another man is anaphoric to a man, but the sense is that the two men should be different, not that they are the same. In other words, while any phrase of the form another $C N$ must, just as an anaphorically used pronoun, find an antecedent in its context of interpretation, the semantic significance of the link is just the opposite here. The DRS for (19) is (20). 


\begin{tabular}{|l|}
\hline $\mathrm{x} \mathrm{y} \mathrm{z}$ \\
\hline $\operatorname{man} \mathrm{x}$ \\
walk-in $\mathrm{x}$ \\
$\mathrm{y} \neq \mathrm{x}$ \\
$\operatorname{man} \mathrm{y}$ \\
$\mathrm{z} \doteq \mathrm{x}$ \\
follow $(\mathrm{y}, \mathrm{z})$ \\
\hline
\end{tabular}

Note that the representation of other-anaphora always needs two reference markers, one introduced by the anaphoric NP itself and one for the antecedent; there is no question here of replacing the former marker by the latter (that is: eliminating the $y$ at the top of (20) and the inequality $y \neq x$ and replacing the other occurrences of $y$ by $x$ ), as that would force the two men to be the same, rather than different. In this regard other-anaphora differs from pronoun anaphora, for which the substitution treatment yields representations that are equivalent to the ones we have been constructing above.

One reason for preferring the treatment of pronoun anaphora we have adopted is that it brings out the similarity as well as the difference between pronouns and phrases with other: In both cases interpretation involves the choice of a suitable antecedent. But the 'links' between the chosen antecedent and the marker for the anaphoric NP are different in nature: they express equality in one case, inequality in the other.

We have said something about the interpretation of three kinds of NPs: indefinite descriptions, anaphoric pronouns and quantified NPs, and we have introduced linking as a central theme in DRT. More about quantification in Section 11. We will now briefly turn to definite descriptions. One of the most obvious facts about them, but a fact systematically ignored or played down in the classical theories of denoting phrases (Frege [29], Russell [58], Strawson [65]), is that, like pronouns, definite descriptions often act as anaphoric expressions.

Indeed, there seems to be a kind of interchangeability in the use of pronouns and descriptions, with a description taking the place of a pronoun in positions where the latter would create an unwanted ambiguity; thus, in discourses like (21) the use of a definite description in the second sentence serves to disambiguate the intended anaphoric link.

(21) A man and a boy came in. The man/he(?) smiled. 
Anaphoric definite descriptions are, like pronouns, linked to existing discourse referents, and thus, like pronouns, they impose certain conditions on the context in which they are used: the context must contain at least one discourse referent that can serve as an antecedent. In this sense both pronouns and anaphoric definite descriptions may be said to carry a certain presupposition: only when the context satisfies this presupposition is it possible to interpret the pronoun, or to interpret the description anaphorically. The descriptive content then serves as information to guide the anaphora resolution process. This will permit anaphora resolution in cases like (21).

Matters are not always this simple, however. Definite descriptions have uses that can hardly be described as anaphoric. For instance, in (22), the description the street is certainly not anaphoric in the strict sense of the word, for there is no antecedent part of the given discourse which has introduced an element that the description can be linked up with.

(22) A man was walking down the street. He was smiling.

It is argued in Heim [35] that the use of a definite description is a means for the speaker to convey that he takes the referent of the description to be in some sense familiar. The hearer who is already acquainted with the street that is intended as the referent of the street by the speaker of (22) may be expected to interpret the description as referring to this street; in such cases speaker and hearer are said to share a common ground (see e.g. Stalnaker [64]) which includes the street in question, and it is this which enables the hearer to interpret the speaker's utterance as he meant it. Such common grounds can also be represented in the form of DRSs. Thus, the common ground just referred to will contain, at a minimum, a component of the form (23), where we assume that the marker $u$ in (23) is anchored to a suitable object (the street that speaker and hearer have in mind).

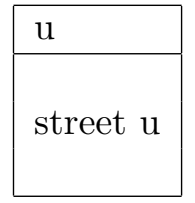

On the assumption of such a 'common ground DRS' (including a suitable anchor) it becomes possible to view the NP the street of (22) as anaphoric. Interpretation of (22) will then be relative to the context DRS (23) and the interpretation of its definite description will yield, by the same principle that governs the interpretation of the man in (21), a DRS like (24). 


\begin{tabular}{|l|}
\hline $\mathrm{u} \mathrm{x} \mathrm{v} \mathrm{y}$ \\
\hline street $\mathrm{u}$ \\
man $\mathrm{x}$ \\
$\mathrm{v} \doteq \mathrm{u}$ \\
street $\mathrm{v}$ \\
was-walking-down $(\mathrm{x}, \mathrm{v})$ \\
$\mathrm{y} \doteq \mathrm{x}$ \\
was-smiling $\mathrm{y}$ \\
\hline
\end{tabular}

This way of dealing with definite descriptions such as the street in (24) may seem to restore uniformity to the analysis of definites. An important difference between definite descriptions and pronouns remains, however. Definite descriptions can be linked much more easily than pronouns to objects that are implicit in the common ground, but have not been explicitly introduced by earlier parts of the same discourse.

To assimilate the use of definite descriptions as unique identifiers (the use that Frege and Russell focus on to the exclusion of all others) to the present anaphoric analysis one must allow for accommodation. When the context available to the hearer does not contain a representation of the referent of a definite description, he may accommodate this context so that it now does contain such a representation, and then proceed as if the representation had been there all along. However, under what conditions precisely accommodation is possible is still a largely unsolved problem.

Interesting cases where the anaphoric account and the unique identification account of definite description have to be combined are the so-called 'bridging descriptions', as in (25) and (26).

(25) (Yesterday) an M.P. was killed. The murderer got away.

(26) Usually when an M.P. is killed, the murderer gets away.

In (25) the murderer is naturally interpreted as referring to the murderer of the M.P. mentioned in the preceding sentence. In other words, the context provides a referent $x$, and the definite description is interpreted as the unique individual who murdered $x$. This account also works for (26), where $x$ varies over murdered M.P.s, and the definite description ranges over the set of unique murderers for all those $x$.

We conclude with a brief remark on proper names. As has been emphasised in the philosophical literature (see in particular Kripke [46]) a proper name 
has no descriptive content, or at any rate its descriptive content plays no essential part in the way it refers. One consequence of this is that a name cannot have more than one referential value (a point which should not be confused with the evident fact that many names-Fred, Fido, John Smith, Fayetteville - are many ways ambiguous). This means that a name cannot have the sort of anaphoric use which we found with the murderer in (25) and (26), and that the antecedent to which the reference marker for a name will have to be linked will always be a marker in the main universe of the context DRS. Logically speaking, therefore, a proper name will always have 'maximally wide scope'. One might think about this process in several ways. One might assume, as in the construction rule for proper names in Kamp [37], that the processing of a proper name always leads to the introduction of a marker in the top DRS, even if the name gets processed in a subordinate DRS somewhere way down. Or one might assume an external element in the semantics of proper names, namely the presence of external anchors: reference markers that are already in place in the top box of a DRS. Any proper name, then, comes equipped with its fixed anaphoric index for linking the name to its anchor. This is the approach we will follow in Section 10.

\section{Discourse Representation Structures}

It is now time to turn to formal details. Let $A$ be a set of constants, and $U$ a set of reference markers or discourse referents (variables, in fact). We also assume that a set of predicate letters with their arities is given. In the following definition, $c$ ranges over $A, v$ over the set $U$, and $P$ over the set of predicates.

\section{Definition 1 (DRSs; preliminary definition)}

terms $t::=v \mid c$

conditions $C::=\top\left|P t_{1} \cdots t_{k}\right| v \doteq t|v \neq t| \neg D$

DRSs $D::=\left(\left\{v_{1}, \ldots, v_{n}\right\},\left\{C_{1}, \ldots, C_{m}\right\}\right)$

Note that this definition of the representation language is provisional; it will be modified in Section 7. We introduce the convention that

$$
\left(\left\{v_{1}, \ldots, v_{n}\right\},\left\{C_{1}, \ldots, C_{m}\right\}\right) \Rightarrow D
$$


is shorthand for

$$
\neg\left(\left\{v_{1}, \ldots, v_{n}\right\},\left\{C_{1}, \ldots, C_{m}, \neg D\right\}\right) .
$$

As in the previous sections DRSs will sometimes be presented in the box notation:

DRSs $D::=$\begin{tabular}{c|}
$v_{1} \cdots v_{n}$ \\
\hline$C_{1}$ \\
$\vdots$ \\
$C_{m}$ \\
\hline
\end{tabular}

The abbreviation $D_{1} \Rightarrow D_{2}$ is rendered in box format by the agreement to write (27) as (28).
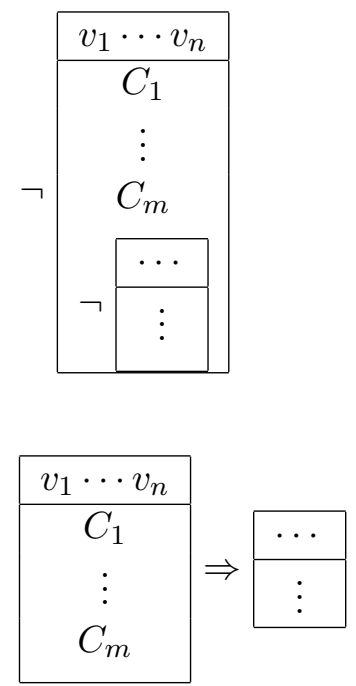

Conditions can be atoms, links, or complex conditions. Complex conditions are negations or implications. As the implications are abbreviations for special negations, we can assume that all complex conditions are negations.

An atom is the symbol $T$ or a predicate name applied to a number of terms (constants or discourse referents), a link is an expression $v \doteq t$ or $v \neq t$, where $v$ is a marker, and $t$ is either a constant or a marker. The clause for complex conditions uses recursion: a complex condition is a condition of the form $\neg D$, where $D$ is a discourse representation structure. 
We will first give a static truth definition for discourse representation structures. Later on, when discussing the problem of compositionality for DRSs, we turn to a context change formulation of those same conditions. Call a first order model $\mathcal{M}=\langle M, I\rangle$ (we assume the domain $M$ is non-empty) an appropriate model for DRS $D$ if $I$ maps the $n$-place predicate names in the atomic conditions of $D$ to $n$-place relations on $M$, the individual constants occurring in the link conditions of $D$ to members of $M$, and (here is the recursive part of the definition) $\mathcal{M}$ is also appropriate for the DRSs in the complex conditions of $D$.

Let $\mathcal{M}=\langle M, I\rangle$ be an appropriate model for DRS $D$. An assignment $s$ for $\mathcal{M}=\langle M, I\rangle$ is a mapping of the set of reference markers $U$ to elements of $M$. The term valuation determined by $\mathcal{M}$ and $s$ is the function $V_{\mathcal{M}, s}$ defined by $V_{\mathcal{M}, s}(t):=I(t)$ if $t \in A$ and $V_{\mathcal{M}, s}(t):=s(t)$ if $t \in U$. In the following definition we use $s[X] s^{\prime}$ for: $s^{\prime}$ agrees with $s$ except possibly on the values of the members of $X$.

\section{Definition 2 (Assignments verifying a DRS)}

An assignment $s$ verifies $D=\left(\left\{v_{1}, \ldots, v_{n}\right\},\left\{C_{1}, \ldots, C_{m}\right\}\right)$ in $\mathcal{M}$ if there is an assignment $s^{\prime}$ with $s\left[\left\{v_{1}, \ldots, v_{n}\right\}\right] s^{\prime}$ which satisfies every member of $\left\{C_{1}, \ldots, C_{m}\right\}$ in $\mathcal{M}$.

\section{Definition 3 (Assignments satisfying a condition)}

1. s always satisfies $\top$ in $\mathcal{M}$.

2. $s$ satisfies $P\left(t_{1}, \ldots, t_{n}\right)$ in $\mathcal{M}$ iff $\left\langle V_{\mathcal{M}, s}\left(t_{1}\right), \ldots, V_{\mathcal{M}, s}\left(t_{n}\right)\right\rangle \in I(P)$.

3. $s$ satisfies $v \doteq t$ in $\mathcal{M}$ iff $s(v)=V_{\mathcal{M}, s}(t)$.

4. $s$ satisfies $v \neq t$ in $\mathcal{M}$ iff $s(v) \neq V_{\mathcal{M}, s}(t)$.

5. $s$ satisfies $\neg D$ in $\mathcal{M}$ iff $s$ does not verify $D$ in $\mathcal{M}$.

Definition 4 Structure $D$ is true in $\mathcal{M}$ if there is an assignment which verifies $D$ in $\mathcal{M}$.

Note that it follows from definition 4 that $(\{x\},\{P x y\})$ is true in $\mathcal{M}$ iff $(\{x, y\},\{P x y\})$ is true in $\mathcal{M}$. In other words: free variables are existentially quantified. 
We leave it to the reader to check that the definition of verifying assignments yields the following requirement for conditions of the form $D_{1} \Rightarrow D_{2}$ :

- $s$ satisfies $D_{1} \Rightarrow D_{2}$ in $\mathcal{M}$, where $D_{1}=\left(X,\left\{C_{1}, \ldots, C_{k}\right\}\right)$, iff every assignment $s^{\prime}$ with $s[X] s^{\prime}$ which satisfies $C_{1}, \ldots, C_{k}$ in $\mathcal{M}$ verifies $D_{2}$ in $\mathcal{M}$.

These definitions are easily modified to take anchors (partial assignments of values to fixed referents) into account. This is done by focusing on assignments extending a given anchor.

It is not difficult to see that the expressive power of basic DRT is the same as that of first order logic. In fact, there is an easy recipe for translating representation structures to formulae of predicate logic. Assuming that discourse referents can do duty as predicate logical variables, the atomic and link conditions of a representation structure are atomic formulae of predicate logic. The translation function ${ }^{\circ}$ which maps representation structures to formulae of predicate logic is defined as follows:

\section{Definition 5 (Translation from DRT to FOL)}

- For DRSs: if $D=\left(\left\{v_{1}, \ldots, v_{n}\right\},\left\{C_{1}, \ldots, C_{m}\right\}\right)$ then $D^{\circ}:=\exists v_{1} \cdots \exists v_{n}\left(C_{1}^{\circ} \wedge \cdots \wedge C_{m}^{\circ}\right)$.

- For atomic conditions (i.e. atoms or links): $C^{\circ}:=C$.

- For negations: $(\neg D)^{\circ}:=\neg D^{\circ}$.

It follows from this that the translation instruction for implications becomes (assume $\left.D_{1}=\left(\left\{v_{1}, \ldots, v_{n}\right\},\left\{C_{1}, \ldots, C_{m}\right\}\right)\right)$

- $\left(D_{1} \Rightarrow D_{2}\right)^{\circ}:=\forall v_{1} \cdots \forall v_{n}\left(\left(C_{1}^{\circ} \wedge \cdots \wedge C_{m}^{\circ}\right) \rightarrow D_{2}^{\circ}\right)$.

The following is now easy to show:

Proposition 6 s verifies $D$ in $\mathcal{M}$ iff $\mathcal{M}, s \models D^{\circ}$, where $=$ is Tarski's definition of satisfaction for first order predicate logic.

It is also not difficult to give a meaning preserving translation from first order predicate logic to basic DRT. In the following definition, $\phi^{\bullet}$ is the DRS corresponding to the predicate logical formula $\phi$, and $\phi_{1}^{\bullet}$ and $\phi_{2}^{\bullet}$ are its first and second components. 


\section{Definition 7 (Translation from FOL to DRT)}

- For atomic formulas: $C^{\bullet}:=(\emptyset, C)$.

- For conjunctions: $(\phi \wedge \psi)^{\bullet}:=\left(\emptyset,\left\{\phi^{\bullet}, \psi^{\bullet}\right\}\right)$.

- For negations: $(\neg \phi)^{\bullet}:=\left(\emptyset, \neg \phi^{\bullet}\right)$.

- For quantifications: $(\exists v \phi)^{\bullet}:=\left(\phi_{1}^{\bullet} \cup\{v\}, \phi_{2}^{\bullet}\right)$.

Proposition $8 \mathcal{M}, s=\phi$ iff $s$ verifies $\phi^{\bullet}$ in $\mathcal{M}$, where $\models$ is Tarski's definition of satisfaction for first order predicate logic.

The difference between first order logic and basic DRT has nothing to do with expressive power but resides entirely in the different way in which DRT handles context. The importance of this new perspective on context and context change is illustrated by the following examples with their DRS representations.

(29) Someone did not smile. He was angry.

(30) Not everyone smiled. *He was angry.

A suitable DRS representation (ignoring tense) for the first sentence of (29) is the following.

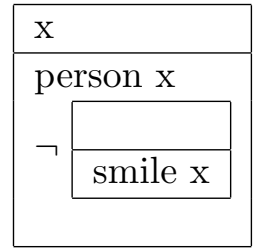

Here we see that the pronoun he in the next sentence of (29) can be resolved by linking it to the marker $x$ occurring in the top box. The anaphoric possibilities of (30) are different, witness its DRS representation (32). 


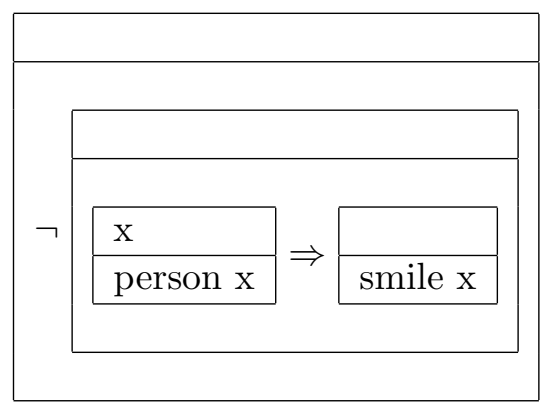

In this case there is no suitable marker available as an antecedent for he in the next sentence of (30).

What we see here is that DRSs with the same truth conditions, such as (31) and (32), may nevertheless be semantically different in an extended sense. The context change potentials of (31) and (32) are different, as the former creates a context for subsequent anaphoric links whereas the latter does not. This is as it should be, of course, as the pronoun in the second sentence of (29) can pick up the reference marker in the first sentence, but the pronoun in the second sentence of (30) cannot. The comparison of (31) and (32) illustrates that meaning in the narrow sense of truth conditions does not exhaust the concept of meaning for DRSs. The extended sense of meaning in which (31) and (32) are different can be informally phrased as follows: (31) creates a new context that can furnish an antecedent for a pronoun is subsequent discourse, (32) does not. This is because (31) changes the context, whereas (32) does not.

\section{The Static and Dynamic Meaning of Represen- tation Structures}

DRT has often been criticized for failing to be 'compositional'. It is important to see what this criticism could mean and to distinguish between two possible ways it could be taken. According to the first of these DRT fails to provide a direct compositional semantics for the natural language fragments to which it is applied. Given the form in which DRT was originally presented, this charge is justifiable, or at least it was so in the past. We will address it in Section 10. In its second interpretation the criticism pertains to the formalism of DRT itself. This objection is groundless. As Definitions 2 and 3 more or less directly imply, the formal language of Definition 1 is 
as compositional as standard predicate logic. We can make the point more explicit by rephrasing Definitions 2 and 3 as a definition of the semantic values $\llbracket \rrbracket_{\mathcal{M}}$ that is assigned to each of the terms, conditions and DRSs of the DRT language by an appropriate model $\mathcal{M}$. As values for DRSs in $\mathcal{M}$ we use pairs $\langle X, F\rangle$ consisting of a finite set of reference markers $X \subseteq U$ and a set of functions $F \subseteq M^{U}$, and as meanings for conditions we use sets of assignments.

\section{Definition 9 (Semantics of DRSs)}

$\llbracket\left(\left\{v_{1}, \ldots, v_{n}\right\},\left\{C_{1}, \ldots, C_{m}\right\}\right) \rrbracket_{\mathcal{M}}:=\left(\left\{v_{1}, \ldots, v_{n}\right\}, \llbracket C_{1} \rrbracket_{\mathcal{M}} \cap \cdots \cap \llbracket C_{m} \rrbracket_{\mathcal{M}}\right)$.

\section{Definition 10 (Semantics of conditions)}

1. $\llbracket P\left(t_{1}, \ldots, t_{n}\right) \rrbracket_{\mathcal{M}}:=\left\{s \in M^{U} \mid\left\langle V_{\mathcal{M}, s}\left(t_{1}\right), \ldots, V_{\mathcal{M}, s}\left(t_{n}\right)\right\rangle \in I(P)\right\}$.

2. $\llbracket v \doteq t \rrbracket_{\mathcal{M}}:=\left\{s \in M^{U} \mid s(v)=V_{\mathcal{M}, s}(t)\right\}$.

3. $\llbracket v \neq t \rrbracket_{\mathcal{M}}:=\left\{s \in M^{U} \mid s(v) \neq V_{\mathcal{M}, s}(t)\right\}$.

4. $\llbracket \neg D \rrbracket_{\mathcal{M}}:=\left\{s \in M^{U} \mid\right.$ for $n o s^{\prime} \in M^{U}: s[X] s^{\prime}$ and $\left.s^{\prime} \in F\right\}$, where $(X, F)=\llbracket D \rrbracket_{\mathcal{M}}$.

To see the connection with the earlier definition of verification, 2 , note that the following proposition holds:

\section{Proposition 11}

- $s$ verifies $D$ in $\mathcal{M}$ iff $\llbracket D \rrbracket_{\mathcal{M}}=\langle X, F\rangle$ and there is an $s^{\prime} \in M^{U}$ with $s[X] s^{\prime}$ and $s^{\prime} \in F$.

- $D$ is true in $\mathcal{M}$ iff $\llbracket D \rrbracket_{\mathcal{M}}=\langle X, F\rangle$ and $F \neq \emptyset$.

If one asks what are the DRS components of a DRS $\left(\left\{v_{1}, \ldots, v_{n}\right\},\left\{C_{1}, \ldots, C_{m}\right\}\right)$, then the answer has to be: there aren't any. For those who do not like this answer, it turns out to be possible to view DRSs as built from atomic building blocks which are also DRSs. This was first pointed out by Zeevat [74]. The DRS language is now given in a slightly different way:

\section{Definition 12 (Building DRSs from atomic DRSs)}


1. If $v$ is a reference marker, $(\{v\}, \emptyset)$ is a DRS.

2. If $(\emptyset,\{\top\})$ is a DRS.

3. If $P$ is an $n$-ary predicate and $t_{1}, \ldots, t_{n}$ are terms, then $\left(\emptyset,\left\{P\left(t_{1}, \ldots, t_{n}\right)\right\}\right)$ is a DRS.

4. If $v$ is a reference marker and $t$ is a term, then $(\emptyset,\{v \doteq t\})$ is a DRS.

5. If $v$ is a reference marker and $t$ is a term, then $(\emptyset,\{v \neq t\})$ is a DRS.

6. If $D$ is a $D R S$, then $(\emptyset, \neg D)$ is a DRS.

7. If $D=(X, C)$ and $D^{\prime}=\left(X^{\prime}, C^{\prime}\right)$ are $D R S s$, then $\left(X \cup X^{\prime}, C \cup C^{\prime}\right)$ is a DRS.

8. Nothing else is a DRS.

It is clear that this defines the same DRS language. Let us use - for the construction step that forms negated DRSs (that is, we use $-D$ for $(\emptyset, \neg D)$ ) and $\oplus$ for the operation of merging the universes and the constraint sets of two DRSs (that is, if $D=(X, C)$ and $D^{\prime}=\left(X^{\prime}, C^{\prime}\right)$, then $D \oplus D^{\prime}:=$ $\left.\left(X \cup X^{\prime}, C \cup C^{\prime}\right)\right)$.

Under this DRS definition, DRSs have become structurally ambiguous. DRS $(\{x\},\{P x, Q x\})$, for example, has several possible construction histories:

- $(\{x\}, \emptyset) \oplus((\emptyset,\{P x\}) \oplus(\emptyset,\{Q x\}))$,

- $(\{x\}, \emptyset) \oplus((\emptyset,\{Q x\}) \oplus(\emptyset,\{P x\}))$,

- $((\{x\}, \emptyset) \oplus(\emptyset,\{P x\})) \oplus(\emptyset,\{Q x\})$,

- and so on.

The DRS semantics to be given next ensures that these structural ambiguities are harmless: the semantic operation corresponding to $\oplus$ is commutative and associative.

The following two semantic operations correspond to the syntactic operations $\oplus,-$ on DRSs (note that we overload the notation by calling the semantic operations by the same names as their syntactic counterparts):

$$
\begin{array}{lll}
\langle X, F\rangle \oplus\langle Y, G\rangle & := & \langle X \cup Y, F \cap G\rangle \\
-\langle X, F\rangle & := & \left\langle\emptyset,\left\{g \in M^{U} \mid \neg \exists f \in F \text { with } g[X] f\right\}\right\rangle
\end{array}
$$

The DRS semantics now looks like this: 


\section{Definition 13}

1. $\llbracket(\{v\}, \emptyset) \rrbracket_{\mathcal{M}}:=\left(\{v\}, M^{U}\right)$.

2. $\llbracket(\emptyset,\{\top\}) \rrbracket_{\mathcal{M}}:=\left(\emptyset, M^{U}\right)$.

3. $\llbracket\left(\emptyset,\left\{P t_{1}, \ldots, t_{n}\right\}\right) \rrbracket_{\mathcal{M}}:=\left(\emptyset,\left\{f \in M^{U} \mid\left\langle V_{\mathcal{M}, f}\left(t_{1}\right), \ldots, V_{\mathcal{M}, f}\left(t_{n}\right)\right\rangle \in\right.\right.$ $I(P)\})$.

4. $\llbracket(\emptyset,\{v \doteq t\}) \rrbracket_{\mathcal{M}}:=\left(\emptyset,\left\{f \in M^{U} \mid f(v)=V_{\mathcal{M}, f}(t)\right\}\right)$.

5. $\llbracket(\emptyset,\{v \neq t\}) \rrbracket_{\mathcal{M}}:=\left(\emptyset,\left\{f \in M^{U} \mid f(v) \neq V_{\mathcal{M}, f}(t)\right\}\right)$.

6. $\llbracket-D \rrbracket_{\mathcal{M}}:=-\llbracket D \rrbracket_{\mathcal{M}}$.

7. $\llbracket D \oplus D^{\prime} \rrbracket_{\mathcal{M}}:=\llbracket D \rrbracket_{\mathcal{M}} \oplus \llbracket D^{\prime} \rrbracket_{\mathcal{M}}$.

Clearly, this provides an elegant and compositional model-theoretic semantics for DRSs. Moreover, it is easily verified that Definition 13 is equivalent to Definitions 9 and 10 in the sense that if $\llbracket D \rrbracket_{\mathcal{M}}=\langle X, F\rangle$, then for any assignment $s, s \in F$ iff $s$ verifies $D$ in $\mathcal{M}$.

The semantics considered so far defines the truth conditions of DRSs. But as we noted at the end of section 5, there is more to the meaning of a DRS than truth conditions alone. For DRSs which define the same truth conditions may still differ in their context change potentials.

To capture differences in context change potential, and not just in truth conditions, we need a different kind of semantics, which makes use of a more finely differentiated (and thus, necessarily, of a more complex) notion of semantic value. There are several ways in which this can be achieved. The one which we follow in the next definition defines the semantic value of a DRS as a relation between assignments - between input assignments, which verify the context to which the DRS is being evaluated, and output assignments, which reflect the way in which the DRS modifies this context. A semantics which characterizes the meaning of an expression in terms of its context change potential is nowadays usually referred to as dynamic semantics, while a semantics like that of the Definitions 2 and 3 or Definitions 9 and 10, whose central concern is with conditions of truth, is called static. The first explicit formulation of a dynamic semantics in this sense can be found in Barwise [7]. An elegant formulation is given in Groenendijk \& Stokhof [33].

Although they are quite different from a conceptual point of view, the dynamic and the static semantics for formalisms like those of DRT are 
nonetheless closely connected. Thus, if we denote the dynamic value of DRS $D$ in model $\mathcal{M}$,-i.e., the relation between assignments of $\mathcal{M}$ which $D$ determines - as ${ }_{s} \llbracket D \rrbracket_{s^{\prime}}^{\mathcal{M}}$, with $s$ the input assignment and $s^{\prime}$ the output assignment, we have:

- If $D=(X, C)$ then: ${ }_{s} \llbracket D \rrbracket_{s^{\prime}}^{\mathcal{M}}$ iff $s[X] s^{\prime}$ and $s^{\prime}$ verifies $D$ in $\mathcal{M}$.

We can also characterize this relation directly, by a definition that is compositional in a similar spirit as Definition 13 in that it characterizes the dynamic value of a complex DRS in terms of the dynamic values of its constituents. It will be convenient to base this definition on a slightly different syntactic characterization of the DRS formalism than we have used hitherto, one in which the symmetric merge of Definition 13 is replaced by an asymmetric merge $\oslash$ defined as follows:

- If $D=(X, C)$ and $D^{\prime}=\left(Y, C^{\prime}\right)$ then $D \oslash D^{\prime}:=\left(X, C \cup C^{\prime}\right)$ is a DRS.

It is clear that all DRSs can be built from atomic DRSs using - and $\oslash$ (but note that $\oslash$ disregards the universe of its second argument).

The dynamic semantics is given as follows. We use ${ }_{s} \llbracket D \rrbracket_{s^{\prime}}^{\mathcal{M}}$ for $s, s^{\prime}$ is an input/output state pair for $D$ in model $\mathcal{M}$, and $s[v] s^{\prime}$ for: $s$ and $s^{\prime}$ differ at most in the value for $v$.

\section{Definition 14}

1. $s \llbracket(\{v\}, \emptyset) \rrbracket_{s^{\prime}}^{\mathcal{M}}$ iff $s[v] s^{\prime}$.

2. $s \llbracket(\emptyset,\{\top\}) \rrbracket_{s^{\prime}}^{\mathcal{M}}$ iff $s=s^{\prime}$.

3. $s_{s} \llbracket\left(\emptyset,\left\{P t_{1}, \ldots, t_{n}\right\}\right) \rrbracket_{s^{\prime}}^{\mathcal{M}}$ iff $s=s^{\prime}$ and $\left\langle V_{\mathcal{M}, s}\left(t_{1}\right), \ldots, V_{\mathcal{M}, s}\left(t_{n}\right)\right\rangle \in I(P)$.

4. $s \llbracket(\emptyset,\{v \doteq t\}) \rrbracket_{s^{\prime}}^{\mathcal{M}}$ iff $s=s^{\prime}$ and $s(v)=V_{\mathcal{M}, s}(t)$.

5. $s \llbracket(\emptyset,\{v \neq t\}) \rrbracket_{s^{\prime \prime}}^{\mathcal{M}}$ iff $s=s^{\prime}$ and $s(v) \neq V_{\mathcal{M}, s}(t)$.

6. ${ }_{s} \llbracket-D \rrbracket_{s^{\prime}}^{\mathcal{M}}$ iff $s=s^{\prime}$ and for no $s^{\prime \prime}$ it is the case that ${ }_{s} \llbracket D \rrbracket_{s^{\prime \prime}}^{\mathcal{M}}$.

7. $s_{s} \llbracket D \oslash D^{\prime} \rrbracket_{s^{\prime}}^{\mathcal{M}}$ iff ${ }_{s} \llbracket D \rrbracket_{s^{\prime}}^{\mathcal{M}}$ and ${ }_{s^{\prime}} \llbracket D^{\prime} \rrbracket_{s^{\prime}}^{\mathcal{M}}$.

The static and the dynamic semantics of DRSs are equivalent, for we have the following proposition: 
Proposition $15 \llbracket D \rrbracket_{\mathcal{M}}=\langle X, F\rangle, s[X] s^{\prime}, s^{\prime} \in F$ iff $s_{s} \llbracket D \rrbracket_{s^{\prime}}^{\mathcal{M}}$.

Still, the relation between static and dynamic semantics that we have given here leaves something to be desired. The composition operations for static semantics and dynamic semantics are different. The basic reason for this is that the dynamic semantics has a notion of sequentiality built in, a notion of processing in a given order. Therefore the commutative merge operation $\oplus$ does not quite fit the dynamic semantics: $\oplus$ is commutative, and sequential merging of DRSs intuitively is not. The operation $\oslash$ is not commutative, but it is unsatisfactory because it discards the dynamic effect of the second DRS (which is treated as if it had an empty universe).

To give a true account of the context change potential of DRSs one has to be able to answer the question how the context change potential of a DRS $D_{1}$ and that of a DRS $D_{2}$ which follows it determine the context change potential of their composition. This leads directly to the question how DRSs can be built from constituent DRSs by an operation of sequential merging.

\section{Sequential Composition of Representation Struc- tures}

Taking unions of universes and constraint sets is a natural commutative merge operation on DRSs, but it is not quite the operation on DRS meanings one would expect, given the dynamic perspective on DRS semantics. Intuitively, the process of gluing an existing DRS representing the previous discourse to a DRS representation for the next piece of natural language text is a process of sequential composition, a process which one would expect not to be commutative.

How should DRS meanings be composed sequentially? Before we address this question, it is convenient to switch to a slightly modified language for DRSs. It turns out that if one introduces a sequencing operator ; the distinction between DRSs and conditions can be dropped. This move yields the following language that we will call the language of proto-DRSs or pDRSs.

$\operatorname{pDRSs} D::=v|\top| P t_{1} \cdots t_{n}|v \doteq t| \neg D \mid\left(D_{1} ; D_{2}\right)$.

In this language, a reference marker taken by itself is an atomic pDRS, and pDRSs are composed by means of ;. Thus, introductions of markers and 
conditions can be freely mixed. Although we drop the distinction between markers and conditions and that between conditions and pDRSs, a pDRS of the form $v$ will still be called a marker, and one of the form $T, P t_{1} \cdots t_{n}$, $v \doteq t$ or $\neg D$ a condition. Thus, a pDRS is a reference marker or an atomic condition or a negation or a ;-composition of pDRSs.

From now on, we will consider $v \neq t$ as an abbreviation of $\neg v \doteq t$, and $D_{1} \Rightarrow D_{2}$ as an abbreviation of $\neg\left(D_{1} ; \neg D_{2}\right)$. It will turn out that the process of merging pDRSs with ';' is associative, so we will often drop parentheses where it does no harm, and write $D_{1} ; D_{2} ; D_{3}$ for both $\left(\left(D_{1} ; D_{2}\right) ; D_{3}\right)$ and $\left(D_{1} ;\left(D_{2} ; D_{3}\right)\right)$.

It is possible to give a commutative semantics for pDRSs, by using the semantic operation - to interpret $\neg$, and $\oplus$ to interpret ;

\section{Definition 16 (Commutative Semantics of pDRSs)}

1. $\llbracket v \rrbracket_{\mathcal{M}}:=\left\langle\{v\}, M^{U}\right\rangle$.

2. $\llbracket \top \rrbracket_{\mathcal{M}}:=\left\langle\emptyset, M^{U}\right\rangle$.

3. $\llbracket P t_{1}, \ldots, t_{n} \rrbracket_{\mathcal{M}}:=\left\langle\emptyset,\left\{f \in M^{U} \mid\left\langle V_{\mathcal{M}, f}\left(t_{1}\right), \ldots, V_{\mathcal{M}, f}\left(t_{n}\right)\right\rangle \in I(P)\right\}\right\rangle$.

4. $\llbracket v \doteq t \rrbracket_{\mathcal{M}}:=\left\langle\emptyset,\left\{f \in M^{U} \mid f(v)=V_{\mathcal{M}, f}(t)\right\}\right\rangle$.

5. $\llbracket \neg D \rrbracket \mathcal{M}:=-\llbracket D \rrbracket_{\mathcal{M}}$.

6. $\llbracket D ; D^{\prime} \rrbracket_{\mathcal{M}}:=\llbracket D \rrbracket_{\mathcal{M}} \oplus \llbracket D^{\prime} \rrbracket_{\mathcal{M}}$.

This interpretation of ; makes merging of pDRSs into a commutative operation. To see the effect of this, look for instance at examples (33) and (34).

(33) A man entered.

(34) A boy smiled.

How should pDRSs for these examples be merged? The commutative merge that we just defined gives the result (35). 


\begin{tabular}{|l|}
\hline $\mathrm{x}$ \\
\hline $\begin{array}{l}\text { man } \mathrm{x} \\
\text { enter } \mathrm{x}\end{array}$
\end{tabular}$;$\begin{tabular}{|l|}
\hline $\mathrm{x}$ \\
\hline $\begin{array}{l}\mathrm{boy} \mathrm{x} \\
\text { smile } \mathrm{x}\end{array}$ \\
\hline
\end{tabular}$=$\begin{tabular}{|l|} 
man $\mathrm{x}$ \\
enter $\mathrm{x}$ \\
boy $\mathrm{x}$ \\
smile $\mathrm{x}$ \\
\hline
\end{tabular}

In the pDRT semantics the two discourse referents for a man and a $a$ boy will be fused, for according to the operation $\oplus$ the fact that a marker is mentioned more than once is irrelevant. This shows that (35) cannot be the right translation of the sequential composition of (33) and (34).

A different approach to merging pDRSs is suggested by the fact that in a dynamic perspective merging in left to right order has a very natural relational meaning:

- $s_{s} \llbracket D_{1} ; D_{2} \rrbracket_{s^{\prime}}^{\mathcal{M}}$ iff there is an assignment $s^{\prime \prime}$ with ${ }_{s} \llbracket D_{1} \rrbracket_{s^{\prime \prime}}^{\mathcal{M}}$ and $s_{s^{\prime \prime}} \llbracket D_{2} \rrbracket_{s^{\prime}}^{\mathcal{M}}$.

This semantic clause complies with the intuition that the first pDRS is interpreted in an initial context $s$ yielding a new context $s^{\prime \prime}$, and this new context serves as the initial context for the interpretation of the second pDRS.

Once we are here a natural way to extend the dynamic approach to the full language suggests itself, as was noted by Groenendijk and Stokhof in [33]. Their observation is basically this. If we interpret the DRS conditions in terms of pairs of assignments, the dynamic semantic values of DRS conditions can be given in the same form as the dynamic values of DRSs.

At first sight, DRS conditions do not look like context changers. If $\left(s, s^{\prime}\right)$ is a context pair for a condition, then always $s=s^{\prime}$, representing the fact that the condition does not change anything. But who cares? If we allow degenerate context changers, we can drop the distinction between conditions and DRSs altogether. What is more, even the distinction between marker introductions and conditions is not essential, for the introduction of a marker $u$ can also be interpreted in terms of context pairs, and the introduction of a list of markers can be obtained by merging the introductions of the components.

These considerations yield the following relational semantics for the pDRS format (this is in fact the semantic format of the dynamic version of first order predicate logic defined in Groenendijk and Stokhof [33]): 


\section{Definition 17 (Relational Semantics of pDRSs)}

1. $s \llbracket v \rrbracket_{s^{\prime}}^{\mathcal{M}}$ iff $s[v] s^{\prime}$.

2. $s \llbracket \top \rrbracket_{s^{\prime}}^{\mathcal{M}}$ iff $s=s^{\prime}$.

3. ${ }_{s} \llbracket P t_{1}, \ldots, t_{n} \rrbracket_{s^{\prime}}^{\mathcal{M}}$ iff $s=s^{\prime}$ and $\left\langle V_{\mathcal{M}, s}\left(t_{1}\right), \ldots, V_{\mathcal{M}, s}\left(t_{n}\right)\right\rangle \in I(P)$.

4. $s \llbracket v \doteq t \rrbracket_{s^{\prime}}^{\mathcal{M}}$ iff $s=s^{\prime}$ and $s(v)=V_{\mathcal{M}, s}(t)$.

5. ${ }_{s} \llbracket \neg D \rrbracket_{s^{\prime}}^{\mathcal{M}}$ iff $s=s^{\prime}$ and for no $s^{\prime \prime}$ it is the case that ${ }_{s} \llbracket D \rrbracket_{s^{\prime \prime}}^{\mathcal{M}}$.

6. ${ }_{s} \llbracket D ; D^{\prime} \rrbracket_{s^{\prime}}^{\mathcal{M}}$ iff there is an $s^{\prime \prime}$ with ${ }_{s} \llbracket D \rrbracket_{s^{\prime \prime}}^{\mathcal{M}}$ and $s_{s^{\prime \prime}} \llbracket D^{\prime} \rrbracket_{s^{\prime}}^{\mathcal{M}}$.

Truth is defined in terms of this, as follows.

Definition 18 (Truth in relational semantics for pDRSs) $D$ is true in $\mathcal{M}$, given $s$, notation $\mathcal{M}, s \models D$, iff there is an $s^{\prime}$ with ${ }_{s} \llbracket D \rrbracket_{s^{\prime}}^{\mathcal{M}}$.

Note that the difference with the previous semantics (definition 16) resides in the interpretation of ; and has nothing to do with with the static/dynamic opposition. To see that, observe that the relational semantics definition 17 can also be given a static formulation. For that, the only change one has to make to definition 16 is in the clause for $D_{1} ; D_{2}$, by interpreting ; as the operation $\circ$ defined as follows:

$$
\langle X, F\rangle \circ\left\langle X^{\prime}, F^{\prime}\right\rangle:=\left\langle X \cup X^{\prime},\left\{f^{\prime} \in F^{\prime} \mid \exists f \in F f\left[X^{\prime}\right] f^{\prime}\right\}\right\rangle
$$

Given this change to definition 16, we have the following proposition:

Proposition $19 \mathcal{M}, s \models D$ iff $\llbracket D \rrbracket=\langle X, F\rangle$ and $\exists f \in F$ with $s[X] f$.

So we see that 17 can be given an equivalent static formulation. Conversely, it is not hard to give a relational clause for $\oplus$ :

$$
f R \oplus S g \Longleftrightarrow f\left[R^{\bullet} \cup S^{\bullet}\right] g \& g \in \operatorname{rng}(R) \cap \operatorname{rng}(S),
$$

where $R^{\bullet}=\{v \in U \mid(f, g) \in R \& f(v) \neq g(v)\}$ (and similarly for $S^{\bullet}$ ).

According to the relational semantics of Definition 17, (36) and (37) have the same meanings. 
(36) $\mathrm{x} ; \mathrm{y}$; man $\mathrm{x}$; woman $\mathrm{y}$; love $(\mathrm{x}, \mathrm{y})$.

(37) $\mathrm{x}$; man $\mathrm{x}$; $\mathrm{y}$; woman $\mathrm{y}$; love $(\mathrm{x}, \mathrm{y})$.

This means that we can use the same box representation (38) for both:

\begin{tabular}{|l|}
\hline $\mathrm{x} \mathrm{y}$ \\
\hline man $\mathrm{x}$ \\
woman $\mathrm{y}$ \\
love $(\mathrm{x}, \mathrm{y})$ \\
\hline
\end{tabular}

Unfortunately, other examples show that the box notation does not really fit the relational semantics for the pDRSs given in definition 17. The use of collecting discourse referents in universes, as it is done in the box format, is that this allows one to see the anaphoric possibilities of a representation at a glance: the discourse referents in the top box are the markers available for subsequent anaphoric linking.

However, when the composition operation ; is interpreted as in Definition 17 (or, alternatively, as the operation o), the pDRS notation becomes capable of expressing distinctions that cannot be captured in the box notation we have been using. Note, for instance that the pDRSs in (39) and (40) are not equivalent with regard to the semantics of Definition 17, although they are equivalent with regard to that given by (the unmodified) Definitions 9 and 10.

(39) $\mathrm{x}$; man $\mathrm{x} ; \operatorname{dog} \mathrm{y} ; \mathrm{y}$; woman $\mathrm{y}$; love $(\mathrm{x}, \mathrm{y})$.

(40) $\mathrm{x} ; \mathrm{y}$; man $\mathrm{x}$; dog $\mathrm{y}$; woman $\mathrm{y}$; love $(\mathrm{x}, \mathrm{y})$.

To take this difference into account the box representation for (39) would have to be something like (41).

\begin{tabular}{|l|l|}
\hline $\mathrm{x}$ & $\mathrm{y}$ \\
\hline $\operatorname{man} \mathrm{x}$ & woman $\mathrm{y}$ \\
$\operatorname{dog} \mathrm{y}$ & love $(\mathrm{x}, \mathrm{y})$ \\
\hline
\end{tabular}


The vertical dividing line in (41) separates the occurrences of $y$ that receive their interpretation from the previously given context from those that are linked to the new introduction.

Thus we see that the relational semantics for pDRSs provides a natural notion of sequential merging, which allows sharing of introduced markers between two DRSs. However, it distinguishes between different introductions of the same marker. This introduces a problem of destructive assignment: after a new introduction of a marker $v$ that was already present, its previous value is lost. This feature of definition 17 is the root cause of the mismatch between box representation and sequential presentation that we just noted. It is also the source of the non-equivalence of the commutative and the relational composition semantics for the pDRS format.

For a fruitful discussion of the problem of sequential merge, it is necessary to be clear about the nature of the different kinds of marker occurrences in a pDRS. In the following discussion we compare the role of reference markers with that of variables in classical logic and in programming languages. Classical logic has two kinds of variable occurrences: bound and free. In the dynamic logic that underlies DRT there are three kinds of variable or marker occurrences (see Visser [70]).

1. marker occurrences that get their reference fixed by the larger context,

2. marker occurrences that get introduced in the current context,

3. markers occurrences that get introduced in a subordinate context.

We will call the first kind fixed marker occurrences, the second kind introduced marker occurrences, and the third kind classically bound marker occurrences. The first kind corresponds roughly to the free variable occurrences of classical logic, and the third kind to the bound variable occurrences of classical logic (hence the name). The second kind is altogether different: these are the markers that embody the context change potential of a given pDRS.

As the distinction between these three kinds of marker occurrences is given by 'dynamic' considerations, it is not surprising that there is a close connection with the various roles that variables can play in imperative programming. Here are the correspondences:

1. Fixed markers correspond to variables in read memory. 
2. Introduced markers correspond to variables in write memory.

3. Bound markers correspond to scratch memory (memory used for intermediate computations that are not part of the output of the program under consideration).

Due to the semantic motivation for this tripartite distinction, the formal definition will depend on the semantics for ; that we adopt. We will give the definition based on the relational semantics.

The set of discourse referents which have a fixed occurrence in a pDRS is given by a function $f i x: p D R S s \rightarrow \mathcal{P} U$. The set of discourse referents which are introduced in a pDRS is given by a function intro $: p D R S s \rightarrow \mathcal{P} U$, and the set of discourse referents which have a classically bound occurrence in a $\mathrm{pDRS}$ is given by a function $c b n d: p D R S s \rightarrow \mathcal{P} U$. To define these functions, we first define a function var on the atomic conditions of a DRS.

$$
\begin{gathered}
\operatorname{var}\left(P t_{1} \cdots t_{n}\right):=\left\{t_{i} \mid 1 \leq i \leq n, t_{i} \in U\right\} \\
\operatorname{var}(v \doteq t):= \begin{cases}\{v, t\} & \text { if } t \in U, \\
\{v\} & \text { otherwise. }\end{cases}
\end{gathered}
$$

\section{Definition 20 (fix, intro, cbnd)}

- $f(x(v):=\emptyset, \operatorname{intro}(v):=\{v\}, \operatorname{cbnd}(v):=\emptyset$.

- $\operatorname{fix}(\top):=\emptyset, \operatorname{intro}(\top):=\emptyset, \operatorname{cbnd}(\top):=\emptyset$.

- $f i x\left(P t_{1} \cdots t_{n}\right):=\operatorname{var}\left(P t_{1} \cdots t_{n}\right), \operatorname{intro}\left(P t_{1} \cdots t_{n}\right):=\emptyset, \operatorname{cbnd}\left(P t_{1} \cdots t_{n}\right):=$ $\emptyset$.

- $\operatorname{fix}(v \doteq t):=\operatorname{var}(v \doteq t), \operatorname{intro}(v \doteq t):=\emptyset, \operatorname{cbnd}(v \doteq t):=\emptyset$.

- $f i x(\neg D):=\operatorname{fix}(D), \operatorname{intro}(\neg D):=\emptyset, \operatorname{cbnd}(\neg D):=\operatorname{intro}(D) \cup \operatorname{cbnd}(D)$.

- $\operatorname{fix}\left(D_{1} ; D_{2}\right):=\operatorname{fix}\left(D_{1}\right) \cup\left(\operatorname{fix}\left(D_{2}\right)-\operatorname{intro}\left(D_{1}\right)\right)$, $\operatorname{intro}\left(D_{1} ; D_{2}\right):=\operatorname{intro}\left(D_{1}\right) \cup \operatorname{intro}\left(D_{2}\right)$, $\operatorname{cbnd}\left(D_{1} ; D_{2}\right):=\operatorname{cbnd}\left(D_{1}\right) \cup \operatorname{cbnd}\left(D_{2}\right)$.

We will occasionally use $\operatorname{activ}(D)$ for the set of markers $f i x(D) \cup \operatorname{intro}(D)$.

The set of conditions of a pDRS is given by the function cond $: p D R S s \rightarrow$ $\mathcal{P}(p D R S s)$, which collects the conditions of $D$ together in a set: 


\section{Definition 21 (cond)}

1. $\operatorname{cond}(v):=\emptyset$.

2. $\operatorname{cond}(\top):=\{\top\}$.

3. $\operatorname{cond}\left(P t_{1} \cdots t_{n}\right):=\left\{P t_{1} \cdots t_{n}\right\}$.

4. $\operatorname{cond}(v \doteq t):=\{v \doteq t\}$.

5. $\operatorname{cond}(\neg D):=\{\neg D\}$.

6. $\operatorname{cond}\left(D_{1} ; D_{2}\right):=\operatorname{cond}\left(D_{1}\right) \cup \operatorname{cond}\left(D_{2}\right)$.

Note that there are pDRSs $D$ with $\operatorname{intro}(D) \cap \operatorname{fix}(D) \neq \emptyset$. An example is given in (42).

$$
P x ; x ; Q x \text {. }
$$

Also, there are pDRSs $D$ where a marker is introduced more than once. An example is given in (43).

(43) $x ; P x ; x ; Q x$

We will call a pDRS proper (or a DRS) if these situations do not occur. Thus, the set of DRSs is defined as follows:

\section{Definition 22 (DRSs)}

- If $v$ is a marker, then $v$ is a DRS.

- $\top$ is a DRS.

- If $t_{1}, \ldots, t_{n}$ are terms and $P$ is an $n$-place predicate letter, then $P t_{1} \cdots t_{n}$ is a $D R S$.

- If $v$ is a marker and $t$ is a term, then $v \doteq t$ is a DRS.

- If $D$ is a DRS, then $\neg D$ is a DRS.

- If $D_{1}, D_{2}$ are DRSs, and $\left(f i x\left(D_{1}\right) \cup \operatorname{intro}\left(D_{1}\right)\right) \cap \operatorname{intro}\left(D_{2}\right)=\emptyset$, then $D_{1} ; D_{2}$ is a DRS. 
- Nothing else is a DRS.

Note that examples (42) and (43) are not DRSs. Indeed, we have:

Proposition 23 For every DRS D, intro $(D) \cap f(x(D)=\emptyset$.

Proposition 23 entails that DRSs of the form $D ; v$ are equivalent to $v ; D$. This means that any DRS $D$ can be written in box format (44) without change of meaning. Indeed, we can view the box format for DRSs as an abstract version of the underlying real syntax.

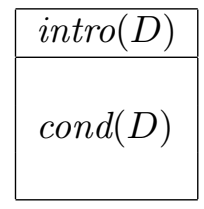

Note that if a DRS $D$ has $\operatorname{intro}(D) \neq \emptyset$ and $\operatorname{cond}(D) \neq \emptyset$, then $D$ must be of the form $D_{1} ; D_{2}$, where $\left(f i x\left(D_{1}\right) \cup \operatorname{intro}\left(D_{1}\right)\right) \cap \operatorname{intro}\left(D_{2}\right)=\emptyset$. We say that $D$ is a simple merge of $D_{1}$ and $D_{2}$.

According to the DRS definition, DRSs are either of one of the forms in (45) or they are simple merges of two DRSs (but note that taking simple merges is a partial operation).
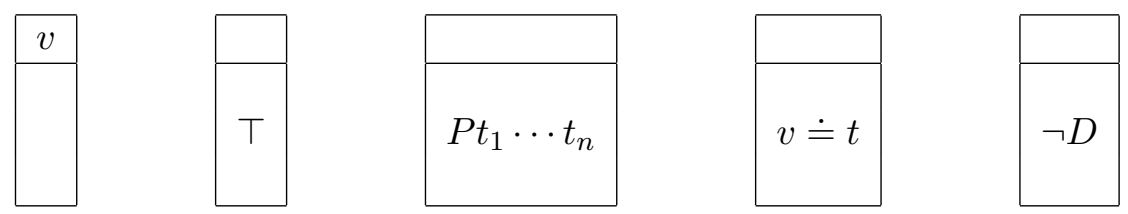

For DRSs, the truth conditions according to the commutative semantics coincide with those according to the relational semantics:

Proposition 24 For all models $\mathcal{M}$, all DRSs D:

$$
\text { if } \llbracket D \rrbracket_{\mathcal{M}}=\langle X, F\rangle \text { then }{ }_{s} \llbracket D \rrbracket_{s^{\prime}}^{\mathcal{M}} \text { iff } s[X] s^{\prime} \quad \text { and } s^{\prime} \in F
$$




\section{Strategies for Merging Representation Structures}

To get a clear perspective on the problem of merging DRSs, note that the issue does not even occur in an approach where a natural language discourse is processed by means of a DRS construction algorithm that proceeds by 'deconstructing' natural language sentences in the context of a given DRS, as in Kamp [37] or Kamp and Reyle [38].

The problem emerges as soon as one modifies this architecture by switching to a set-up where representations for individual sentences are constructed first, and next these have to be merged in left to right order. Suppose we want to construct a DRS for the sequential composition of $S_{1}$ and $S_{2}$ on the basis of a DRS $D_{1}$ for $S_{1}$ and a DRS $D_{2}$ for $S_{2}$. Now it might happen that $D_{1} ; D_{2}$ is not a DRS, because $\left(f i x\left(D_{1}\right) \cup \operatorname{intro}\left(D_{1}\right)\right) \cap \operatorname{intro}\left(D_{2}\right) \neq \emptyset$. Our idea is to resolve this situation by applying a renaming strategy. In the example sentences given so far the problem has been avoided by a prudent choice of indices, but example (46) would pose such a conflict.

(46) A man $^{1}$ entered. A boy ${ }^{1}$ smiled.

The initial representation for the sequential composition of $D_{1}$ and $D_{2}$ can be given by $D_{1} \bullet D_{2}$. The problem of sequential merge now takes the form of finding strategies for reducing DRS-like expressions with occurrences of - to DRSs.

Before we list of a number of options for 'merge reduction', we define a class of reducible DRSs or RDRSs (assume $D$ ranges over DRSs):

RDRSs $R::=D|\neg R|\left(R_{1} \bullet R_{2}\right)$.

Thus, RDRSs are compositions out of DRSs by means of $\neg$ and $\bullet$. It is useful to extend the definitions of intro, fix and cbnd to RDRSs:

\section{Definition 25 (fix, intro, cbnd for RDRSs)}

- $\operatorname{fix}(\neg R):=\operatorname{fix}(R), \operatorname{intro}(\neg R):=\emptyset, \operatorname{cbnd}(\neg R):=\operatorname{intro}(R) \cup \operatorname{cbnd}(R)$.

- $f i x\left(R_{1} \bullet R_{2}\right):=\operatorname{fix}\left(R_{1}\right) \cup\left(f i x\left(R_{2}\right)-\operatorname{intro}\left(R_{1}\right)\right)$, $\operatorname{intro}\left(R_{1} \bullet R_{2}\right):=\operatorname{intro}\left(R_{1}\right) \cup \operatorname{intro}\left(R_{2}\right)$, $\operatorname{cbnd}\left(R_{1} \bullet R_{2}\right):=\operatorname{cbnd}\left(R_{1}\right) \cup \operatorname{cbnd}\left(R_{2}\right)$. 
We use $\bullet$ for sequential merge. The various options for how to merge DRSs all have a semantic and a syntactic side, for they must handle two questions:

1. What is the semantics of $\bullet$ ?

\section{How can RDRSs be reduced to DRSs?}

In order to talk about these reductions in a sensible way, we must take negative context into account. Here is a definition of negative contexts $(D$ ranges over DRSs, $R$ over RDRSs).

Negative Contexts $N::=\neg \square|\neg N|(N ; D)|(D ; N)|(N \bullet R) \mid(R \bullet N)$.

Condition on $(N ; D)$ : $\operatorname{activ}(N) \cap \operatorname{intro}(D)=\emptyset$. Condition on $(D ; N)$ : $\operatorname{activ}(D) \cap \operatorname{intro}(N)=\emptyset$, where $\operatorname{activ}(N)$ and $\operatorname{intro}(N)$ are calculated on the basis of intro $(\square):=\operatorname{fix}(\square):=\operatorname{cbnd}(\square):=\emptyset$.

What the definition says is that a negative context is an RDRS with one constituent RDRS immediately within the scope of a negation replaced by $\square$. If $N$ is a negative context, then $N[R]$ is the result of substituting RDRS $R$ for $\square$ in $N$. The definition of negative contexts allows us to single out an arbitrary negated sub-RDRS $R$ of a given RDRS by writing that RDRS in the form $N[R]$.

Contexts $C::=\square \mid N$.

A context is either a $\square$ or a negative context. If $C$ is a context, then $C[R]$ is the result of substituting RDRS $R$ for $\square$ in $N$. Thus, if we want to say that a reduction rule applies to an RDRS $R$ that may (but need not) occur immediately within the scope of a negation sign within a larger RDRS, we say that the rule applies to $C[R]$. If we specify a reduction rule

$$
R \Longrightarrow R^{\prime}
$$

this is meant to be understood as licensing all reductions of the form:

$$
C[R] \longrightarrow C\left[R^{\prime}\right]
$$

This format ensures that the rule can both apply at the top level and at a level bounded by a negation sign inside a larger RDRS. 
We will now discuss several options for merge reduction: symmetric merge, prudent merge, destructive merge, deterministic merge with substitution, and indeterministic merge with substitution.

Symmetric Merge Interpret $\bullet$ as $\oplus$ and ; as ०. The reduction rules that go with this are:

$$
\begin{aligned}
(R \bullet v) & \Longrightarrow(v ; R) \\
(R \bullet \top) & \Longrightarrow(R ; \top) \\
\left(R \bullet P t_{1}, \ldots, t_{n}\right) & \Longrightarrow\left(R ; P t_{1}, \ldots, t_{n}\right) \\
\left(R \bullet \neg R^{\prime}\right) & \Longrightarrow\left(R ; \neg R^{\prime}\right) \\
\left((R \bullet v) \bullet R^{\prime}\right) & \Longrightarrow\left((v ; R) \bullet R^{\prime}\right) \\
\left((R \bullet \top) \bullet R^{\prime}\right) & \Longrightarrow\left((R ; \top) \bullet R^{\prime}\right) \\
\left(\left(R \bullet P t_{1}, \ldots, t_{n}\right) \bullet R^{\prime}\right) & \Longrightarrow\left(\left(R ; P t_{1}, \ldots, t_{n}\right) \bullet R^{\prime}\right) \\
\left(\left(R \bullet \neg R_{1}\right) \bullet R_{2}\right) & \Longrightarrow\left(\left(R ; \neg R^{\prime}\right) \bullet R_{2}\right) \\
\left(R \bullet\left(R_{1} ; R_{2}\right)\right) & \Longrightarrow\left(\left(R \bullet R_{1}\right) \bullet R_{2}\right) \\
\left(R \bullet\left(R_{1} \bullet R_{2}\right)\right) & \Longrightarrow\left(\left(R \bullet R_{1}\right) \bullet R_{2}\right)
\end{aligned}
$$

Partial Merge Interpret $\bullet$ as a partial operation (see e.g. Muskens [50]) while retaining $\circ$ as the interpretation of ; (as we will do throughout the remainder of this section). To give the semantics, we have to take context into account. Assume that the semantics of a DRS $D$ is given as a triple $\langle X, Y, F\rangle$, where $X=f i x(D), Y=\operatorname{intro}(D)$ and $F$ is a set of assignments, then the following partial operation gives the semantics of partial merge:

$\langle X, Y, F\rangle \odot\left\langle X^{\prime}, Y^{\prime}, F^{\prime}\right\rangle:= \begin{cases}\left\langle X \cup X^{\prime}, Y \cup Y^{\prime}, F \cap F^{\prime}\right\rangle & \text { if }(X \cup Y) \cap Y^{\prime}=\emptyset, \\ \uparrow & \text { otherwise. }\end{cases}$

The reduction rules that go with this: same as above, except for the following change in the rules that handle marker introductions:

$$
\begin{aligned}
(R \bullet v) & \Longrightarrow(R ; v) \text { if } v \notin f i x(R) \cup \text { intro }(R) \\
(R \bullet v) & \Longrightarrow \text { ERROR if } v \in f i x(R) \cup \text { intro }(R) \\
\left((R \bullet v) \bullet R^{\prime}\right) & \Longrightarrow\left((R ; v) \bullet R^{\prime}\right) \text { if } v \notin f i x(R) \cup \text { intro }(R) \\
\left((R \bullet v) \bullet R^{\prime}\right) & \Longrightarrow \text { ERROR if } v \in f i x(R) \cup \text { intro }(R) .
\end{aligned}
$$

Prudent Merge To give the semantics of prudent merging for $\bullet$ (see Visser [70]), one again has to take context fully into account.

$$
\langle X, Y, F\rangle \odot\left\langle X^{\prime}, Y^{\prime}, F^{\prime}\right\rangle:=\left\langle X \cup\left(X^{\prime}-Y\right), Y \cup\left(Y^{\prime}-X\right), F \cap F^{\prime}\right\rangle .
$$


Reduction rules that go with this: same as above, except for the following change in the rules that handle marker introduction:

$$
\begin{aligned}
(R \bullet v) & \Longrightarrow(R ; v) \text { if } v \notin f i x(R) \cup \operatorname{intro}(R) \\
(R \bullet v) & \Longrightarrow R \text { if } v \in f i x(R) \cup \text { intro }(R) \\
\left((R \bullet v) \bullet R^{\prime}\right) & \left.\Longrightarrow(R ; v) \bullet R^{\prime}\right) \text { if } v \notin f i x(R) \cup \text { intro }(R) \\
\left((R \bullet v) \bullet R^{\prime}\right) & \Longrightarrow R \bullet R^{\prime} \text { if } v \in f i x(R) \cup \text { intro }(R) .
\end{aligned}
$$

Destructive Merge Interpret $\bullet$ as $\circ$ (relational composition), and allow destructive assignment. The reduction rule that goes with this is very simple: replace all occurrences of $\bullet$ in one go by ;, and interpret ; as ०. But of course, this reduction does not yield DRSs but only proto-DRSs.

For the next two perspectives on merging DRSs, we need to develop a bit of technique for handling substitution, or, more precisely, marker renamings.

Definition 26 A marker renaming is a function $\theta: U \rightarrow U$, such that its domain $\operatorname{Dom}(\theta):=\{v \in U \mid v \neq \theta(v)\}$ is finite. If $\theta$ is a renaming with $\operatorname{Dom}(\theta)=\left\{v_{1}, \ldots, v_{n}\right\}$, then $\operatorname{Rng}(\theta):=\left\{\theta\left(v_{1}\right), \ldots, \theta\left(v_{n}\right)\right\}$. A renaming $\theta$ avoids a set $X \subseteq U: \Leftrightarrow R n g(\theta) \cap X=\emptyset$. If $\theta$ is a renaming, then $\theta-v:=$ the renaming $\sigma$ that is like $\theta$ but for the fact that $\sigma(v)=v$. If $X \subseteq U$ then $\theta X:=\{\theta(x) \mid x \in X\}$. A marker renaming $\theta$ is injective on $X: \Leftrightarrow$ $|X|=|\theta X|$.

We will refer to a renaming $\theta$ with domain $\left\{v_{1}, \ldots, v_{n}\right\}$ as $\left[\theta\left(v_{1}\right) / v_{1}, \ldots, \theta\left(v_{n}\right) / v_{n}\right]$. Thus, $[x / y]$ is the renaming $\theta$ with $\theta(u)=x$ if $u=y$ and $\theta(u)=u$ otherwise. This renaming is of course injective on $\{x\}$, but not on $\{x, y\} .[x / y, x / z]$ is a renaming which is not injective on $\{y, z\}$. $[x / y, x / z]-z=[x / y]$.

A renaming of a subset of intro $(D)$ intuitively has as its semantic effect that the write memory of $D$ gets shifted. Renaming in a dynamic system like DRT works quite differently from variable substitution in classical logic, because of the three kinds of marker occurrences that have to be taken into account: fix, intro and cbnd. In particular, a renaming of intro $(D)$ has to satisfy the following requirements:

1. it should be injective on intro $(D)$,

2. it should avoid fix $(D)$, 
3. it should leave $\operatorname{cbnd}(D)$ untouched.

The first two of these requirements can be imposed globally. Requirement (iii) should be part of the definition of the effects of renamings on (R)DRSs: we will handle it by distinguishing between outer and inner renaming. For an outer renaming of RDRS $R$ with $\theta$ we employ $\theta R$, for an inner renaming $\bar{\theta} R$. Inner renaming is renaming within a context where marker introductions act as classical binders, i.e., within the scope of an occurrence of $\neg$. For example, if $\theta=[v / x, w / y]$, then:

$$
\theta(x ; \neg(y ; R x y))=v ; \neg(y ; R v y) .
$$

A renaming $\theta$ induces functions from terms to terms as follows:

$$
\theta(t):= \begin{cases}\theta(v) & \text { if } t=v \text { with } v \in U \\ t & \text { if } t \in C .\end{cases}
$$

A renaming $\theta-v$ induces functions from terms to terms as follows:

$$
\theta-v(t):= \begin{cases}\theta(w) & \text { if } t=w \neq v \text { with } w \in U, \\ v & \text { if } t=v, \\ t & \text { if } t \in C .\end{cases}
$$

The induced renaming functions from (R)DRSs to (R)DRSs are given by:

$$
\begin{aligned}
\theta v & :=\theta(v) \\
\theta \top & :=\top \\
\bar{\theta} \top & :=\top \\
\theta\left(P t_{1} \cdots t_{n}\right) & :=P \theta t_{1} \cdots \theta t_{n} \\
\bar{\theta}\left(P t_{1} \cdots t_{n}\right) & :=P \theta t_{1} \cdots \theta t_{n} \\
\theta(v \doteq t) & :=\theta v \doteq \theta t \\
\bar{\theta}(v \doteq t) & :=\theta v \doteq \theta t \\
\theta(\neg R) & :=\neg \bar{\theta} R \\
\bar{\theta}(\neg R) & :=\neg \bar{\theta} R \\
\theta(v ; R) & :=\theta v ; \theta R \\
\bar{\theta}(v ; R) & :=v ; \bar{\theta}-v R \\
\theta(C ; R) & :=\theta C ; \theta R, \quad C \in\left\{P t_{1} \cdots t_{n}, v \doteq t, \neg R^{\prime}\right\} \\
\bar{\theta}(C ; R) & :=\bar{\theta} C ; \bar{\theta} R, \quad C \in\left\{P t_{1} \cdots t_{n}, v \doteq t, \neg R^{\prime}\right\} \\
\theta\left(\left(R_{1} ; R_{2}\right) ; R_{3}\right) & :=\theta\left(R_{1} ;\left(R_{2} ; R_{3}\right)\right) \\
\bar{\theta}\left(\left(R_{1} ; R_{2}\right) ; R_{3}\right) & :=\bar{\theta}\left(R_{1} ;\left(R_{2} ; R_{3}\right)\right),
\end{aligned}
$$


plus rules for $\bullet$ exactly like those for ;

For the semantics, let us again assume that a meaning for DRS $D$ is a triple $\langle X, Y, F\rangle$, where $X=f i x(D), Y=\operatorname{intro}(D)$, and $F$ is the set of assignments satisfying $\operatorname{cond}(D)$.

Definition $27 \theta$ is a proper renaming for $D R S D: \Leftrightarrow$

1. $\operatorname{Dom}(\theta) \subseteq \operatorname{intro}(D)$,

2. $\theta$ is injective on intro $(D)$,

3. $\operatorname{Rng}(\theta) \cap \operatorname{fix}(D)=\emptyset$.

Definition 28 If $F \subseteq M^{U}, \theta F:=\left\{g \in M^{U} \mid g \circ \theta \in F\right\}$.

For example, if $F=\left\{f \in M^{U} \mid f(x) \in I(P)\right\}$, and $\theta=[y / x]$, then:

$$
[y / x] F=\left\{g \in M^{U} \mid g \circ[y / x](x) \in I(P)\right\}=\left\{g \in M^{U} \mid g(y) \in I(P)\right\} .
$$

Proposition 29 If $\theta$ is a proper renaming for $D$ and $|D|^{\mathcal{M}}=\langle X, Y, F\rangle$ then $|\theta D|^{\mathcal{M}}=\langle X, \theta Y, \theta F\rangle$.

The upshot if this proposition is that a proper renaming only changes the write memory of a DRS.

Deterministic Merge With Substitution The sequence semantics for dynamic predicate logic defined in Vermeulen [67] can be used as a semantics for a language of unreduced DRSs:

$$
R::=\mathrm{PUSH} v|\top| P t_{1} \cdots t_{n}|v \doteq t| \neg R \mid\left(R_{1} \bullet R_{2}\right),
$$

where $v$ ranges over a set $U$ of markers without indices. The meaning of a variable introduction $v$ in sequence semantics is: push a new value for $v$ on a stack of $v$ values. Clearly, this prevents the destructive use of memory that we saw in connection with definition 17. Suggestive notation for this: PUSH $v$.

We can reduce expressions of this language to a language of proper DRSs where the markers are taken from the set of indexed markers $U^{\prime}:=\left\{u_{i} \mid\right.$ 
$u \in U, i>0\}$. The corresponding merge reduction rules for this use fully determined renamings, as follows.

First we do a global renaming, by replacing every occurrence of $v \in U$, except those immediately preceded by a PUSH, by $v_{1} \in U^{\prime}$. Next, assume that we are in a situation $D \bullet$ PUSH $v \bullet R$, where $D$ is a DRS (no occurrences of PUSH in $D$, no occurrences of $\bullet$ in $D$ ). Then there are two cases to consider.

It may be that $v_{j}$ does not occur in $f i x(D) \cup \operatorname{intro}(D)$, for any index $j$. In that case, rewrite as follows:

$$
(D \bullet \operatorname{PUSH} v) \bullet R \Longrightarrow\left(D ; v_{1}\right) ; R \text {. }
$$

It may also be that $v_{j}$ does occur in $f(x(D) \cup \operatorname{intro}(D)$, for some index $j$. In that case, let $i$ be $\sup \left(\left\{j \in \mathbb{N} \mid v_{j} \in f i x(D) \cup\right.\right.$ intro $\left.\left.(D)\right\}\right)$, and rewrite as follows:

$$
(D \bullet \operatorname{PUSH} v) \bullet R \Longrightarrow\left(D ; v_{i+1}\right) ;\left[v_{i+1} / v_{i}\right] R .
$$

The idea behind these instructions is that if $v_{j}$ does not occur in $D$, then $v_{1}$ can safely be introduced, and it will actively bind the occurrences of $v_{1}$ which occur in open position on the right. If $v_{j}$ does occur in $D$, then the present push should affect the $v$-variables with the highest index in open position on the right. This is precisely what the renaming $\left[v_{i+1} / v_{i}\right]$ effects.

Indeterministic Merge With Substitution Indeterministic merge does involve a family $\odot_{\theta}$ of merge operations, where $\theta$ is a renaming that is constrained by the two DRSs $D_{1}$ and $D_{2}$ to be merged, in the sense that $\theta$ is proper for $D_{2}$ and $\theta$ avoids the set intro $\left(D_{1}\right) \cup f i x\left(D_{1}\right)$. If the interpretations of $D_{1}$ and $D_{2}$ are given by $\left\langle X_{1}, Y_{1}, F_{1}\right\rangle$ and $\left\langle X_{2}, Y_{2}, F_{2}\right\rangle$, respectively, then the interpretation of $D_{1} \bullet_{\theta} D_{2}$ is given by:

$$
\left\langle X_{1} \cup X_{2}, Y_{1} \cup \theta Y_{2}, F_{1} \cap \theta F_{2}\right\rangle .
$$

If $\theta$ is constrained in the way stated above this is a proper DRS denotation. The rules for indeterministic merge reduction use renamings, as follows (we 
use $\operatorname{activ}(R)$ for $\operatorname{intro}(R) \cup f i x(R))$ :

$$
\begin{aligned}
& (R \bullet v) \Longrightarrow \begin{cases}(R ; v) & \text { if } v \notin \operatorname{activ}(R) \\
(R ; w) & \text { if } v \in \operatorname{activ}(R) \\
& w \notin \operatorname{activ}(R)\end{cases} \\
& (R \bullet \top) \Longrightarrow(R ; \top) \\
& \left(R \bullet P t_{1}, \ldots, t_{n}\right) \Longrightarrow\left(R ; P t_{1}, \ldots, t_{n}\right) \\
& \left(R \bullet \neg R^{\prime}\right) \quad \Longrightarrow \quad\left(R ; \neg R^{\prime}\right) \\
& \left((R \bullet v) \bullet R^{\prime}\right) \Longrightarrow \begin{cases}\left((R ; v) ; R^{\prime}\right) & \text { if } v \notin \operatorname{activ}(R), \\
\left((R ; w) ;[w / v] R^{\prime}\right. & \text { if } v \in \operatorname{activ}(R), \\
& w \notin \operatorname{activ}(R) \cup \operatorname{activ}\left(R^{\prime}\right)\end{cases} \\
& \left((R \bullet \top) \bullet R^{\prime}\right) \quad \Longrightarrow \quad\left((R ; \top) \bullet R^{\prime}\right) \\
& \left(\left(R \bullet P t_{1}, \ldots, t_{n}\right) \bullet R^{\prime}\right) \Longrightarrow\left(\left(R ; P t_{1}, \ldots, t_{n}\right) \bullet R^{\prime}\right) \\
& \left(\left(R \bullet \neg R_{1}\right) \bullet R_{2}\right) \quad \Longrightarrow \quad\left(\left(R ; \neg R_{1}\right) \bullet R_{2}\right) \\
& \left(R \bullet\left(R_{1} ; R_{2}\right)\right) \quad \Longrightarrow \quad\left(\left(R \bullet R_{1}\right) \bullet R_{2}\right) \\
& \left(R \bullet\left(R_{1} \bullet R_{2}\right)\right) \quad \Longrightarrow \quad\left(\left(R \bullet R_{1}\right) \bullet R_{2}\right)
\end{aligned}
$$

Note that under the indeterministic merge regime, $\bullet$ does not get an independent semantics, so one cannot talk about 'the' meaning of $D \bullet D^{\prime}$ anymore, only about its meaning modulo renaming of intro $\left(D^{\prime}\right)$. One can still prove that different reductions of $R$ to normal form (i.e. to proper DRSs) are always write variants of one another, i.e., $R \rightarrow D$ and $R \rightarrow D^{\prime}$ together entail that there is some proper renaming $\theta$ of $D$ with $\theta D=D^{\prime}$.

A set of RDRSs together with a set of merge reduction rules like the example sets given above is a so-called abstract reduction system (Klop [45]), and the theory of abstract reduction systems can fruitfully be applied to their study (Van Eijck [19]). What all merge reduction rule sets above, with the exception of destructive merge, have in common is that they start out from reducible DRSs and produce proper DRSs as normal forms. They all take into account that the merge operation $\bullet$ should not destroy anaphoric links. Merge with substitution has as an additional feature that it preserves anaphoric sockets, and that is what we will use in the sequel. For practical reasons we opt for the indeterministic version, to avoid possible confusion due to the appearance of a new kind of indices (indicating stack depth).

Each RDRS or DRS has a set of anaphoric plugs and a set of anaphoric sockets. The plugs anchor the representation structure to previous discourse or 
to contextually given antecedents. In both reduced and unreduced RDRSs, these plugs have fixed names, given by $f i x(R)$. The sockets are the anchoring ground for the next bit of discourse. In unreduced RDRSs, the sockets do not have fixed names yet, and they may not yet represent the full set of anaphoric possibilities of the represented discourse. During the process of merge reduction, the internal wiring of the representation structure gets re-shuffled and some members of $\operatorname{intro}(R)$ may end up with a new name, to make room for extra sockets. If $D$ is a fully reduced DRS, however, the sockets have fixed names, given by $\operatorname{intro}(D) \cup f i x(D)$, and this set of markers represents the full set of anaphoric possibilities for subsequent discourse.

Here is a concrete example of how disjoint merging according to the indeterministic merge regime works:

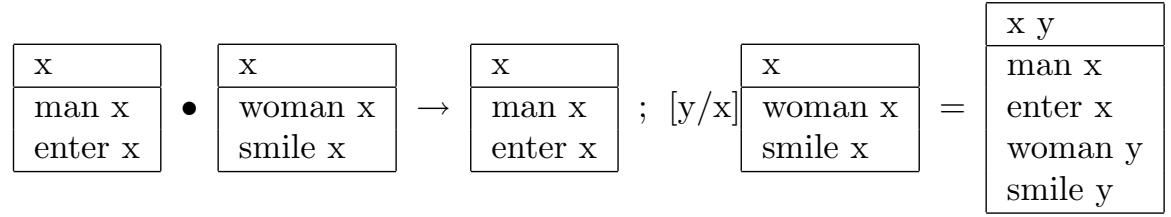

In DRT with indeterministic merge, introduced markers are always new, so no information is ever destroyed, and merging of representations preserves all anaphoric possibilities of the parts that are merged.

We now know what the basic building blocks of DRT are, namely structures as given in (45), and what is the glue that puts them together, namely the disjoint merge operation involving marker renaming. This concludes the discussion of compositionality for DRSs. Quite a few philosophical and technical questions concerning the natural notion of information ordering in DRT remain. See Visser [69] for illumination on these matters.

\section{Disjoint Merge and Memory Management}

Reference markers are similar to variables, but differ from them in that they are not bound by logical operators in the usual sense. In fact, reference markers behave more like variables in programming languages than like variables in ordinary first order logic (Section 7 above).

Anaphoric links are created by linking new reference markers to available ones. How does one discard references? By de-allocating storage space on 
popping out of a 'subroutine'. The representation, in box format, for (3) is given in (48).

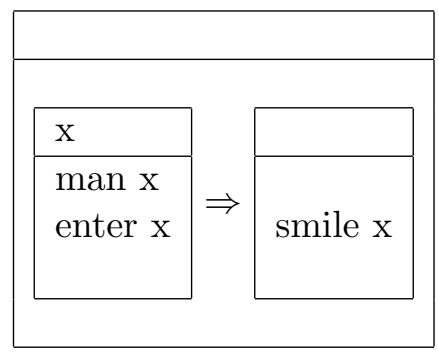

The semantic treatment of this uses a subroutine for checking if every way of making a reference to a man who enters (where the reference is established via marker $x$ ) makes the property given by the consequent of the clause succeed. Next the storage space for $x$ is de-allocated, which explains why an anaphoric link to a man in subsequent discourse is ruled out, or at least infelicitous (see example (49)).

(49) If a $\operatorname{man}^{1}$ enters, he 1 smiles. ${ }^{*} \mathrm{He}_{1}$ is happy.

Thus we see that anaphoric linking is not subsumed under variable binding, or at least not under variable binding perceived in a standard fashion, as in first order logic. The process is much more akin to variable binding in programming, where storage space is created and discarded dynamically, and where links to a variable remain possible until the space occupied by the variable gets de-allocated to be used for something else, so that further anaphoric links remain possible as long as the variable space for the antecedent remains accessible.

Reference markers, as we have seen, are allocated pieces of storage space for (representations of) things in the world. We can picture the building of a representation structure as an interactive process, where we give instructions to make memory reservations and to provide names for the allocated chunks of memory, as in (50).

(50) new(Var)

The system responds by allocating a chunk of memory of the correct size and by returning a name as value of Var, say $u 385$, indicating that a piece 
of storage space is allocated and henceforth known under the name $u 385$, where 385 presumably is the offset from the beginning of the piece of memory where the representation under construction is stored. Once storage space has been allocated to a discourse referent, it is useful to know the scope of the allocation. In DRT the scope of the introduction of a discourse referent is closed off by the closest $\neg$ operator (or the closest $\Rightarrow$ operator, in case $\Rightarrow$ is taken as a primitive) that has that introduction in its scope.

Of course, this interactive picture is an inside picture of what happens during the representation building process. We must also be able to look at the situation from the outside, and answer the question what happens if we assume that we have built and stored two representation structures $D_{1}, D_{2}$ in the memory of a computer, one after the other. Next, we want to store them in memory simultaneously, i.e., to merge them, where the merging has to preserve sequential order. This will in general involve changing the names of those variables declared in the second representation that would otherwise overwrite the area of memory already used by the first representation.

What if some very suspicious semanticist still has qualms about disjoint merge because of the indeterminism of the operation? We then would have to explain to him (or her) that the indeterminism is entirely natural, as it reflects the fact that the renaming operation is nothing but the familiar operation of copying variable values to a different (unused) part of memory before combining two memory states (Figure 1). Disjoint merge is indeter-

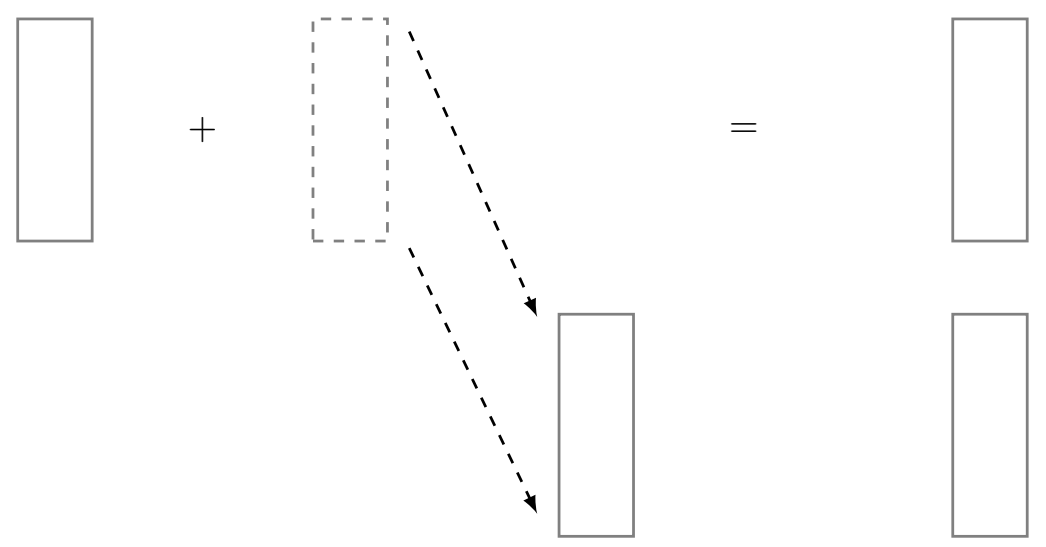

Figure 1: Copying registers before merging memory states.

ministic simply because any way of copying part of memory to a safe new 
location will do. This suggests that indeterminism is a strength rather than a weakness of the disjoint merge.

The story of a reasonable definition of merge is a story of memory management. Assuming we have an unlimited supply of memory available, we may picture the data part of memory where the active markers of representation structure $D$ reside as an array $a[0], \ldots, a[i], \ldots$, where the $a[i]$ are the cells containing the referents (pointers to the individuals in the model under consideration). Where exactly in absolute memory representation structure $D$ is stored is immaterial; we assume it is stored in relative memory, that is to say, at some unknown offset $m$ from the start of the data part of memory. If the marker set $\operatorname{activ}(D)$ of structure $D$ occupies $k$ memory cells and is stored at offset $m$ from the beginning of data memory, then the active markers of $D$ range from $a[m]$ to $a[m+k]$.

As soon as we are willing to keep track of where in relative memory the result of merging representation structures $D_{1}$ and $D_{2}$ is going to reside, counting from the offset where $D_{1}$ is stored, a deterministic disjoint merge is readily available, in terms of a particular renaming $\theta$ determined by the memory locations. Now the story gets us down to the level of programming the bare silicon of the discourse representation machine, so to speak. Assuming the markers $\operatorname{activ}\left(D_{1}\right)$ of $D_{1}$ reside in memory at $u[0], \ldots, u[i]$ (where $u[0]=$ $a[m]$, for some offset $m$ ), and the markers $\operatorname{activ}\left(D_{2}\right)$ of $D_{2}$ reside in some scratch part of memory $s[0], \ldots, s[j]$, then $D_{1}$ and $D_{2}$ can be merged after a renaming $\theta=[u[i+1] / s[0], \ldots, u[i+j+1] / s[j]]$, and $\operatorname{activ}\left(D_{1} ; \theta D_{2}\right)$ will reside in memory at $u[0], \ldots, u[i+j+1]$.

But once again, such a detailed description of the implementation of merge is really unnecessary. What we will need for the next section is the assumption that for all $R_{1}, R_{2}$, the merge $R_{1} \bullet R_{2}$ is a well-defined (reducible) discourse representation structure, and that the result of merging $R_{1}$ and $R_{2}$ is independent of the choice of marker names, in the sense that the operation does not destroy anaphoric sockets due to variable name clashes. This is precisely what we have got in the definition of the merge operation provided by indeterministic merge. What it all boils down to is this. Anaphoric links are essentially arrows pointing from anaphoric expressions to antecedents (Figure 2). Often these links can be represented by indices, as in (51).

(51) $\mathrm{John}^{i}$ hates a man' ${ }^{j}$ who hates $\operatorname{him}_{i}$ and another $\operatorname{man}_{j}$ who does not.

The actual choice of the index numbers does not matter. What matters is 


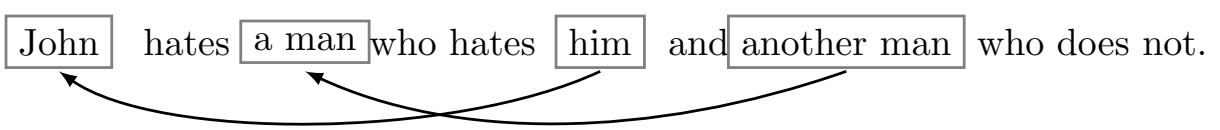

Figure 2: Anaphoric links are arrows.

the property of having the same index. In a slogan: anaphoric arrows are index pairs $\left(\begin{array}{c}i \\ \end{array}{ }_{i}\right)$ modulo renamings. Of course, one might also assume that all indices have been picked appropriately from the start, but as a general strategy this would seem quite unrealistic; and in any case the point we want to make here is that that assumption is not necessary.

$$
\frac{v}{\text { data }}
$$

Figure 3: Direct allocation of storage space to variable $v$.

While we are at the topic of memory management, we might as well mention that there are at least two non-equivalent ways in which storage space for reference markers can get allocated. In the first variant, which we have assumed until now, on allocating memory and giving it a name $v, v$ becomes the name of the piece of memory containing the data (Figure 3).

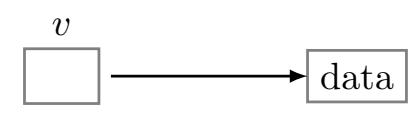

Figure 4: Indirect allocation of storage space to variable $v$.

In the second variant, $v$ refers to the data indirectly by pointing to a piece of storage space containing the data. This second variant allows much greater versatility in manipulating data structures. The name $v$ might for instance be used to allocate and point to a new piece of memory, without destroying previous data (Figure 5). Indirect allocation ensures that old data are preserved in memory, although they may no longer be accessible under the old name (Figure 5). The development of a pointer semantics for DRT suggests the use of pointer stacks to keep track of referents that are contextually salient, allowing pointers to be set to nil to indicate that a referent has drifted out of focus, and so on. For a detailed account of a pointer 


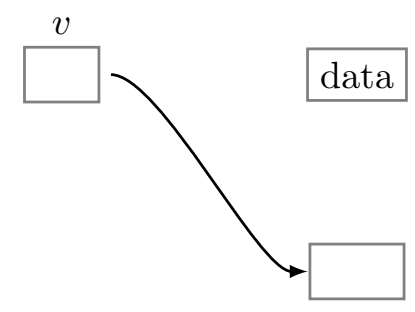

Figure 5: Allocating new memory space to $v$ without destroying old data.

semantics for a variant of DRT we refer the reader to Vermeulen [68].

\section{Constructing DRSs for Natural Language Frag- ments}

As we have seen in Section 6, there is one sense in which the compositionality of DRT is unproblematic: the representation formalisms DRT proposes are as compositional as one could like. In fact, all semantic definitions we have considered in the last three sections, from Definition 9, onwards, have been essentially compositional: they either were, or else could readily be converted into, compositional definitions of the semantic values that expressions of these formalisms determine in a model. Moreover, in the last two sections we have looked at a number of merge operations for putting two DRSs together into a single one. These operations too, we found, can be given direct semantic interpretations which map the semantic values of the component DRSs into the semantic value of the compound.

But what about compositionality in the second sense? Does DRT provide a way of analyzing fragments of natural language which assigns these fragments a semantics that is compositional with respect to these fragments themselves, a semantics that is compositional with respect to a natural syntax for these fragments? The original formulation of DRT did not seem to provide such an analysis, and it was even suggested at the time that a compositional treatment of the natural language fragments then considered would be impossible. In the meantime we have, through the dynamic reformulation of DRT discussed in Sections 7, 8 and 9, come to see that such pessimism is not quite warranted: when applied judiciously, the traditional computational methods familiar from Montague Grammar can be made to 
work so that they assign sentences and texts from these fragments the same truth conditions as the original version of DRT. It suffices to define the building blocks of DRSs as suitably typed expressions of a typed language. In particular, each word of the natural language fragment in question can be assigned an expression of the typed language as its lexical entry, and these expressions can then be combined, by 'semantic' rules corresponding to syntactic composition rules, into representations of any given sentence or text of the fragment; by an entirely analogous process, one can compute the semantic value of the sentence or text directly from the semantic values of the (entries of) the words composing them.

Whether the compositional approach towards DRT, which operates under much stricter constraints than the original DRT approach (e.g. Kamp \& Reyle [38]), can handle all the purposes to which DRT has been put is a question to which there is at present no clear answer. We turn to this question briefly at the end of this section and again in Section 12.

A DRS construction algorithm for a given natural language fragment has to provide instructions for extending a given DRS with the information contained in a sentence from the fragment. This entails that the processing instructions for that sentence should take information from the previous representation into account. In practice, this is the list of available referents. Assuming that the representation of the previous discourse is in reduced form, we may take it that we have a list $u_{1}, \ldots, u_{n}$ available of reference markers introduced by previous discourse. Pronouns may be resolved to any member of this list, and also to markers that get introduced by antecedents in the sentence under consideration.

The process of anaphoric resolution on the basis of available information from the representation of previous discourse poses a highly non-trivial challenge, and it is questionable if a real algorithm for this process is on the cards. The following problem is more manageable. Assuming that an anaphoric indexing for a sentence is given, and also that a decision has been made about the relative scopes of the operators (i.e., a reading of the sentence has been fixed by the sentence grammar), give an algorithm for updating an available representation structure with the information from that sentence. In fact, as we shall see, we get a lot of this for free because of the presence of the merge operation $\bullet$.

To illustrate the process of constructing DRSs for natural language fragments, we begin by defining a sentence grammar for a toy fragment. Basic 


\begin{tabular}{|c|c|}
\hline category & abbreviates \\
\hline $\mathrm{CN}$ & $\mathrm{S} / \mathrm{E}(*, * *)$ \\
\hline $\mathrm{VP}(*)$ & $\mathrm{E}\left(\operatorname{Nom},{ }^{*}, *\right) \backslash \mathrm{S}$ \\
\hline $\mathrm{NP}($ case $, \mathrm{i}, \mathrm{j})$ & $\mathrm{S} /(\mathrm{E}($ case $, \mathrm{i}, \mathrm{j}) \backslash \mathrm{S})$ \\
\hline TV(tense) & $\mathrm{VP}($ tense $) / \mathrm{NP}\left(\mathrm{Acc}^{*},{ }^{*}\right)$ \\
\hline $\operatorname{DET}(\mathrm{i}, \mathrm{j})$ & $\mathrm{NP}(*, \mathrm{i}, \mathrm{j}) / \mathrm{CN}$ \\
\hline AUX & $\mathrm{VP}($ Tensed $) / \mathrm{VP}(\operatorname{Inf})$ \\
\hline REL & $(\mathrm{CN} \backslash \mathrm{CN}) / \mathrm{VP}($ Tensed $)$ \\
\hline
\end{tabular}

Figure 6: Category abbreviations for a toy grammar.

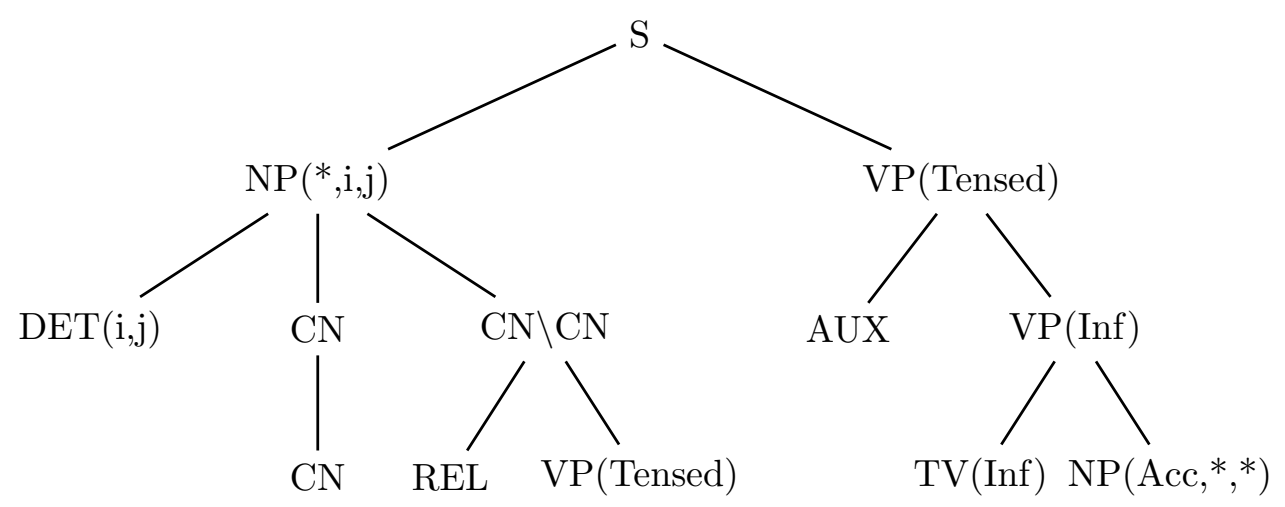

Figure 7: Example of a possible sentence structure according to the toy grammar. 
categories are $\mathrm{S}$ (without features) for sentences, TXT (without features) for texts, and E (with features for case, antecedent index i, anaphoric index j), for markers for individual entities. We assume the category abbreviations given in Figure 6. Here the feature variable tense ranges over the values Tensed and Inf, the feature variable case ranges over the values Nom and $A c c$, and the index features range over the positive natural numbers. The example structure generated by this grammar given in Figure 7 illustrates how the grammar works. Further information about the categorial format with feature unification is provided in the Chapters on Categorial Grammar and on Feature Structures in this Handbook.

\begin{tabular}{|c|c|c|c|}
\hline expression & category & translates to & type \\
\hline $\mathrm{a}^{i}$ & $\operatorname{DET}\left(\mathrm{i},{ }^{*}\right)$ & $\lambda P \lambda Q\left(u_{i} \bullet P\left(u_{i}\right) \bullet Q\left(u_{i}\right)\right)$ & $((\mathrm{e}, \mathrm{T}),((\mathrm{e}, \mathrm{T}), \mathrm{T}))$ \\
\hline every $^{i}$ & $\operatorname{DET}(\mathrm{i}, *)$ & $\lambda P \lambda Q \neg\left(\left(u_{i} \bullet P\left(u_{i}\right)\right) \bullet \neg Q\left(u_{i}\right)\right)$ & $((\mathrm{e}, \mathrm{T}),((\mathrm{e}, \mathrm{T}), \mathrm{T}))$ \\
\hline no ${ }^{i}$ & $\operatorname{DET}\left(\mathrm{i},{ }^{*}\right)$ & $\lambda P \lambda Q \neg\left(\left(u_{i} \bullet P\left(u_{i}\right)\right) \bullet Q\left(u_{i}\right)\right)$ & $((\mathrm{e}, \mathrm{T}),((\mathrm{e}, \mathrm{T}), \mathrm{T}))$ \\
\hline $\operatorname{another}_{j}^{i}$ & $\operatorname{DET}(\mathrm{i}, \mathrm{j})$ & $\lambda P \lambda Q\left(u_{i} ; u_{i} \neq u_{j} \bullet P\left(u_{i}\right) \bullet Q\left(u_{i}\right)\right)$ & $((\mathrm{e}, \mathrm{T}),((\mathrm{e}, \mathrm{T}), \mathrm{T}))$ \\
\hline the $_{j}^{i}$ & $\operatorname{DET}(\mathrm{i}, \mathrm{j})$ & $\lambda P \lambda Q\left(u_{i} ; u_{i} \doteq u_{j} \bullet P\left(u_{i}\right) \bullet Q\left(u_{i}\right)\right)$ & $((\mathrm{e}, \mathrm{T}),((\mathrm{e}, \mathrm{T}), \mathrm{T}))$ \\
\hline $\operatorname{his}_{j}^{i}$ & $\operatorname{DET}(\mathrm{i}, \mathrm{j})$ & $\lambda P \lambda Q\left(u_{i} ;\right.$ poss $\left.\left(u_{j}, u_{i}\right) \bullet P\left(u_{i}\right) \bullet Q\left(u_{i}\right)\right)$ & $((\mathrm{e}, \mathrm{T}),((\mathrm{e}, \mathrm{T}), \mathrm{T}))$ \\
\hline $\mathrm{Bill}_{i}$ & $\mathrm{NP}(*, *, i)$ & $\lambda P\left(u_{i} \doteq b \bullet P\left(u_{i}\right)\right)$ & $((\mathrm{e}, \mathrm{T}), \mathrm{T})$ \\
\hline who & REL & $\lambda P \lambda Q \lambda v(Q(v) \bullet P(v))$ & $((\mathrm{e}, \mathrm{T}),((\mathrm{e}, \mathrm{T}),(\mathrm{e}, \mathrm{T})))$ \\
\hline $\mathrm{he}_{i}$ & $\mathrm{NP}\left(\right.$ nom, $\left.{ }^{*}, \mathrm{i}\right)$ & $\lambda P\left(P\left(u_{i}\right)\right)$ & $((\mathrm{e}, \mathrm{T}), \mathrm{T})$ \\
\hline $\operatorname{him}_{i}$ & $\mathrm{NP}\left(\mathrm{acc},{ }^{*}, \mathrm{i}\right)$ & $\lambda P\left(P\left(u_{i}\right)\right)$ & $((\mathrm{e}, \mathrm{T}), \mathrm{T})$ \\
\hline $\operatorname{man}$ & $\mathrm{CN}$ & $\lambda v(\operatorname{man}(v))$ & $(\mathrm{e}, \mathrm{T})$ \\
\hline boy & & $\lambda v($ boy $(v))$ & $(\mathrm{e}, \mathrm{T})$ \\
\hline smiles & VP(Tensed) & $\lambda v($ smile $(v))$ & $(\mathrm{e}, \mathrm{T})$ \\
\hline smile & VP(Inf) & $\lambda v($ smile $(v))$ & $(\mathrm{e}, \mathrm{T})$ \\
\hline has & TV(Tensed) & $\lambda \mathcal{P} \lambda u(\mathcal{P} \lambda v($ poss $(u, v)))$ & $(((\mathrm{e}, \mathrm{T}), \mathrm{T}),(\mathrm{e}, \mathrm{T}))$ \\
\hline have & $\mathrm{TV}(\mathrm{Inf})$ & $\lambda \mathcal{P} \lambda u(\mathcal{P} \lambda v($ poss $(u, v)))$ & $(((\mathrm{e}, \mathrm{T}), \mathrm{T}),(\mathrm{e}, \mathrm{T}))$ \\
\hline hates & TV(Tensed) & $\lambda \mathcal{P} \lambda u(\mathcal{P} \lambda v($ hate $(u, v)))$ & $(((\mathrm{e}, \mathrm{T}), \mathrm{T}),(\mathrm{e}, \mathrm{T}))$ \\
\hline hate & TV(Inf) & $\lambda \mathcal{P} \lambda u(\mathcal{P} \lambda v($ hate $(u, v)))$ & $(((\mathrm{e}, \mathrm{T}), \mathrm{T}),(\mathrm{e}, \mathrm{T}))$ \\
\hline does not & AUX & $\lambda P \lambda v \neg P(v))$ & $((\mathrm{e}, \mathrm{T}),(\mathrm{e}, \mathrm{T}))$ \\
\hline & $(\mathrm{S} / \mathrm{S}) / \mathrm{S}$ & $\lambda p \lambda q(\neg(p \bullet \neg q))$ & $(\mathrm{T},(\mathrm{T}, \mathrm{T}))$ \\
\hline . & $\mathrm{S} \backslash(\mathrm{TXT} / \mathrm{S})$ & $\lambda p \lambda q(p \bullet q)$ & $(\mathrm{T},(\mathrm{T}, \mathrm{T}))$ \\
\hline . & $\mathrm{TXT} \backslash(\mathrm{TXT} / \mathrm{S})$ & $\lambda p \lambda q(p \bullet q)$ & $(\mathrm{T},(\mathrm{T}, \mathrm{T}))$ \\
\hline
\end{tabular}

Figure 8: Lexical component of the toy fragment for English.

If we start out with basic types $e$ for entities and $T$ for state transitions (not truth values!), then the table given in Figure 8 defines the lexical component of a tiny fragment of English. Variables $u, v$ range over type $e$, variables $p, q$ over type $T$, variables $P, Q$ over type $(e, T)$, variables $\mathcal{P}$ over type $((e, T), T)$.

We distinguish between variables of the typed logic and reference markers (i.e. variables of the dynamic representation). Markers $u_{i}$ are taken from a 
set $U$ which we assume to be disjoint from the set $V_{e}$ of variables of type $e$. Thus, from the perspective of the typed logic the reference markers behave like constants. A rather straightforward definition of the interpretation of a typed expression can now be given in terms of an interpretation function $I$, a (typed logic) variable assignment $g$, and a marker assignment $f$. This theme is played (sometimes with minor variations) in Asher [4], Bos et al. [12], Kuschert [47] and Muskens [50].

From the point of view of the dynamic logic reference markers are variables, to be sure, but, as we have seen, substitution for dynamic variables is handled quite differently from variable substitution in static logics. Another way of expressing the relation between typed variables and reference markers is by saying that $\beta$ reduction (which affects typed variables) and merge reduction (which affects markers) are orthogonal: there is no interaction between the $\lambda$ reduction rules and the $\bullet$ reduction rules.

The category table in the lexicon makes clear that example sentence (52) has the structure specified in Figure 7.

(52) The man who smiles does not hate Bill.

Some other sentences in the fragment are given in (53) and (54) (we use the particular nouns and verbs in the table as paradigms, of course).

(53) If a man hates Bill, he does not smile.

(54) If a bishop meets another bishop, he blesses him.

For convenience, we have assumed that the connective '?' serves as a discourse constructor. Example (55) gives a text which is in the fragment.

(55) The man who smiles does not hate Bill. He respects Bill.

Note that $\bullet$ is used for merging of structures in all those cases where renaming may still be necessary. The translations of if and every use $\neg(p \bullet \neg q)$ rather than $p \Rightarrow q$ to allow for the possibility of renaming during the merge of the components.

The composition of representation structures for these example sentences is a matter of routine. See Gamut [30] for a didactic account of the general procedure, Asher [4] and Muskens [50] for applications in dynamic semantics, 
and Bouchez, Van Eijck and Istace [13] for a description of an implementation of dynamic semantics using the technique.

As an example, let us go through the procedure of building a representation for (55). We assume the following indexing to indicate the intended anaphoric link.

(56) The $[\text { man who smiles }]^{1}$ does not hate Bill. $\mathrm{He}_{1}$ respects Bill.

We also have to choose anaphoric indices for the man who smiles and Bill. Assume these to be 2 and 3 , respectively. In the table we find translation $\lambda P \lambda Q \lambda v(Q(v) \bullet P(v))$ for who, while smiles translates as $\lambda v($ smile $(v))$. These combine by functional application, which gives (57) (after renaming of variables for perspicuity).

$$
\lambda Q \lambda v(Q(v) \bullet \lambda w(\text { smile }(w))(v))
$$

Expression (57) $\beta$ reduces to (58).

(58) $\lambda Q \lambda v(Q(v) \bullet$ smile $(v))$.

Combining (58) with the translation of man, we get (59).

$$
\lambda v(\lambda w(\operatorname{man}(w))(v) \bullet \text { smile }(v)) .
$$

Expression (59) $\beta$ reduces to (60).

(60) $\lambda v(\operatorname{man}(v) \bullet$ smile $(v))$.

Combining (60) with the translation of the $e_{2}^{1}$ gives expression (61) as translation for the $e_{2}^{1}$ man who smiles:

(61) $\lambda Q\left(u_{1} ; u_{1} \doteq u_{2} \bullet \lambda w(\operatorname{man}(w) \bullet\right.$ smile $\left.(w))\left(u_{1}\right) \bullet Q\left(u_{1}\right)\right)$.

Applying $\beta$ reduction to expression (61) gives (62).

(62) $\lambda Q\left(u_{1} ; u_{1} \doteq u_{2} \bullet \operatorname{man}\left(u_{1}\right) \bullet\right.$ smile $\left.\left(u_{1}\right) \bullet Q\left(u_{1}\right)\right)$.

In a similar way, we get (63) for does not hate Bill 3 . 


$$
\lambda u \neg\left(u_{3} \doteq b \bullet \text { hate }\left(u, u_{3}\right)\right)
$$

Combining (62) and (63) gives the translation of the first sentence of (56):

$$
\left(u_{1} ; u_{1} \doteq u_{2} \bullet \operatorname{man}\left(u_{1}\right) \bullet \text { smile }\left(u_{1}\right) ; \neg\left(u_{3} \doteq b \bullet \text { hate }\left(u_{1}, u_{3}\right)\right)\right) \text {. }
$$

Merge reduction of (64) (with the identical renaming) gives:

$$
\left(u_{1} ; u_{1} \doteq u_{2} ; \text { man }\left(u_{1}\right) ; \text { smile }\left(u_{1}\right) ; \neg\left(u_{3} \doteq b ; \text { hate }\left(u_{1}, u_{3}\right)\right)\right) \text {. }
$$

\begin{tabular}{|c|c|}
\hline \multicolumn{2}{|l|}{$u_{1}$} \\
\hline \multicolumn{2}{|c|}{$\begin{array}{l}u_{1} \doteq u_{2} \\
\text { man } u_{1} \\
\text { smile } u_{1}\end{array}$} \\
\hline$\neg$ & $\begin{array}{l}u_{3} \doteq b \\
\text { hate }\left(u_{1}, u_{3}\right)\end{array}$ \\
\hline
\end{tabular}

In box format:

The translation of the second sentence of (56) is (67).

$$
\left(u_{3} \doteq b \bullet \operatorname{respect}\left(u_{1}, u_{3}\right)\right)
$$

One merge reduction step, with identical renaming:

$$
\left(u_{3} \doteq b ; \text { respect }\left(u_{1}, u_{3}\right)\right)
$$

The translation of discourse (56) is the result of applying the semantic operation for text composition (the semantics for '?' in the lexicon table) to (65) and (67), in that order:

(69) $\lambda p \lambda q(p \bullet q))\left(u_{1} ; u_{1} \doteq u_{2} ;\right.$ man $\left(u_{1}\right) ;$ smile $\left(u_{1}\right) ; \neg\left(u_{3} \doteq b\right.$; hate $\left.\left.\left(u_{1}, u_{3}\right)\right)\right)$ $\left(u_{3} \doteq b ;\right.$ respect $\left.\left(u_{1}, u_{3}\right)\right)$.

Two $\beta$ reductions and one further merge reduction with identical renaming gives the following result (in box format): 


\begin{tabular}{|c|c|}
\hline \multicolumn{2}{|l|}{$u_{1}$} \\
\hline \multicolumn{2}{|c|}{$\begin{array}{l}u_{1} \doteq u_{2} \\
\text { man } u_{1} \\
\text { smile } u_{1}\end{array}$} \\
\hline$\neg$ & $\begin{array}{l}u_{3} \doteq b \\
\text { hate }\left(u_{1}, u_{3}\right)\end{array}$ \\
\hline $\begin{array}{l}u_{3} \\
\text { res }\end{array}$ & $\begin{array}{l}=b \\
\text { ect }\left(u_{1}, u_{3}\right)\end{array}$ \\
\hline
\end{tabular}

The fact that no new discourse referent gets introduced for the proper name Bill is a reflection of our treatment of proper names. Here is the entry for proper names in the lexicon table again:

\begin{tabular}{|l|l|l|l|}
\hline expression & category & translates to & type \\
\hline Bill $_{i}$ & $\mathrm{NP}\left({ }^{*},{ }^{*}, \mathrm{i}\right)$ & $\lambda P\left(u_{i} \doteq b \bullet P\left(u_{i}\right)\right)$ & $((\mathrm{e}, \mathrm{T}), \mathrm{T})$ \\
\hline
\end{tabular}

Here $i$ is the index that links the constant $b$ for the proper name to its external anchor. Anaphoric links involving proper names are insensitive to where the name gets introduced, for they are interpreted as links where the anaphor and the proper name are both anaphoric expressions with a common 'externally given' antecedent.

At this point a couple of remarks are in order about the rules of index assignment which are part of our present treatment. The first remark concerns the lower indices, which, we have been assuming, must be assigned not only to pronouns but in fact to definite noun phrases of any kind. The requirement that every definite NP must receive a lower index reflects the so-called familiarity principle (see Heim [35]), according to which a definite NP is used felicitously only when the utterance context already contains a reference marker for its referent, which can then serve as 'anaphoric antecedent' for the NP. It is doubtful that the familiarity principle can be upheld in as rigid and comprehensive a form as this, in which it is taken to apply to every occurrence of every type of definite noun phrase. The definite description the man who smiles in (52) is a case in point. It would certainly be possible to use this phrase for picking out from a given crowd the unique person smiling, pretty much as many philosophers, from Frege and Russell onwards, have been claiming about definite descriptions. Such a use could easily occur in a context in which no reference marker for the smiling man had as yet 


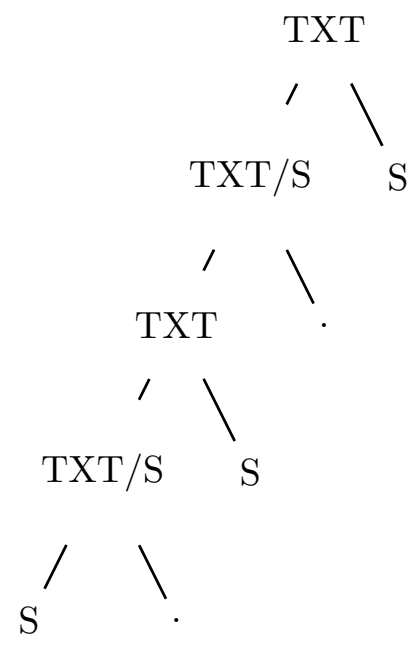

Figure 9: The structure of a three sentence text in our grammar set-up.

been introduced. A treatment of definite descriptions which insists on the presence of antecedent reference markers for definites could still be saved by assuming that definite descriptions always come with a presupposition that the context contains such a reference marker, but that this presupposition can be easily accommodated when necessary. One may have one's doubts about the plausibility of this rescue strategy. But even if we go along with it, we will have to reformulate our semantics in such a way that it allows for such accommodations, and allows them to be made at those points where human interpreters would have to make them. In other words, the theory will have to be restated so that it can deal with aspects of presupposition. Unfortunately, this is a matter that we cannot go into for reasons of space. For the treatment of presupposition within DRT, see the bibliographical remarks in section E.

A similar remark is in order about the lower indices of proper names such as John. Does the use of a proper name presuppose that its referent is already represented in the given context? Perhaps, but if so, then 'context' needs to be construed in a quite liberal way. So, before such a treatment of proper names can be considered satisfactory, much more needs to be said about how the notion of context is to be construed - what kinds of information may contexts include, from what kinds of contexts can their information 
come, etc.

The second remark concerns the implicit assumption that the texts to which our theory is applied come fully equipped with all the necessary upper and lower indices and that all of these have been assigned in advance. One way in which this assumption gets us into difficulties shows up in the text (71), which has the structure indicated in Figure 9.

(71) A man ${ }^{1}$ who mistrusted the assistant ${ }_{3}^{2}$ walked in. $\mathrm{He}_{1}$ asked for the manager ${ }_{4}^{2}$. $\mathrm{He}_{2}$ turned out to be on holiday.

As the text structure indicates, first representations are built for the first two sentences and these are merged together, and only then is a representation for the third sentence merged with the representation of the preceding discourse. Note that in this case the merge of the representations of the first and the second sentence would involve a renaming of the discourse referent for the manager, to avoid a clash with the marker for the assistant from the first sentence. This means that the anaphoric index 2 in the third sentence is not going to pick up a reference to the manager anymore, as was presumably intended.

The example points towards an aspect of DRT that deserves comment. DRT - this is as true of the form in which it was originally stated as it is of the dynamic formulation presented here - is not a theory of anaphora resolution: the theory itself tells us little about how to select the intended antecedent for a given anaphoric expression from among a number of possible candidates. The only substantive contribution which classical DRT makes to the problem of anaphora resolution consists in what it has to say about the 'accessibility' of reference markers that have been introduced in one part of a text to anaphoric expressions occurring elsewhere (see e.g. Kamp \& Reyle [38], Ch. 1.4); but this is only a small part of a comprehensive account of anaphora resolution capable of predicting the intended anaphoric connections in all cases in which these are evident to a human interpreter.

Arguably this is as it should be. It would be unreasonable to demand of a theory of linguistic semantics - and it is that which DRT originally aimed at - that it incorporate a detailed account of anaphora resolution, which would have to rely on a host of pragmatic principles as well as on an indefinite amount of world knowledge.

It seems not unreasonable, however, to demand of such a theory that it offer a 
suitable interface to other (pragmatic and/or extra-linguistic) components of a comprehensive theory of meaning which are designed to deal with anaphora resolution (see Sidner [63], Webber [73], and Chapter 10 of Alshawi c.s. [2]) and to allow these other components to come into action at those points when the information needed for anaphora resolution has become available and the resolution is necessary for interpretation to proceed. To insist that all upper and lower indexation take place in advance of interpretation would fly in the face of this demand. For as a rule it is only through and thus after interpretation of the earlier parts of a discourse that the correct links for subsequent anaphoric expressions can be established.

\section{The Proper Treatment of Quantification in DRT}

As we have seen above, universal quantification can be treated in terms of $D \Rightarrow D^{\prime}$, which can in turn be taken as an abbreviation of $\neg\left(D ; \neg D^{\prime}\right)$. Look again at the treatment of the quantifiers every and no in the fragment given above.

\begin{tabular}{|l|l|l|l|}
\hline expression & category & translates to & type \\
\hline every $^{i}$ & $\mathrm{DET}\left(\mathrm{i},{ }^{*}\right)$ & $\lambda P \lambda Q \lambda c \mapsto\left(u_{i} ; P i\right) \Rightarrow Q i$ & $K \rightarrow K \rightarrow T$ \\
no $^{i}$ & $\mathrm{DET}\left(\mathrm{i},{ }^{*}\right)$ & $\lambda P \lambda Q \lambda c \mapsto \neg\left(u_{i} ; P i ; Q i\right)$ & $K \rightarrow K \rightarrow T$ \\
\hline
\end{tabular}

Working out an example like Every man walks on the basis of this gives a representation that is equivalent to the following box notation:

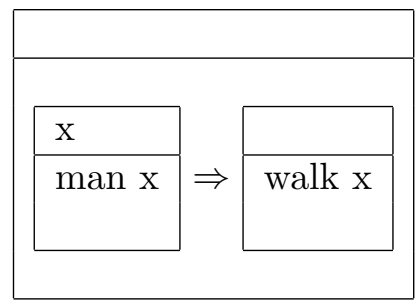

The treatment of every creates the impression that the quantificational force resides in the dynamic implication $\Rightarrow$. Note, by the way, that all occurrences of marker $x$ in representation (72) are classically bound. The same holds for more complex examples like the representation for (73) in (74).

(73) Every man who meets a nice woman smiles at her. 


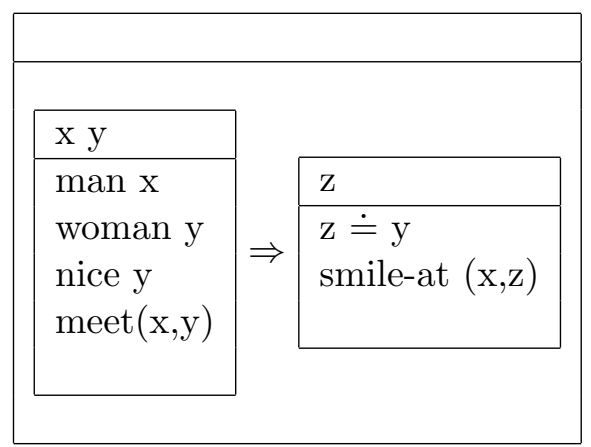

Now consider sentence (75).

(75) Most men who meet a nice woman smile at her.

This sentence is true if most individuals which satisfy the descriptive content of the subject NP also satisfy the VP, i.e. if most men who meet a nice woman have the property of smiling at her. Note that assessing the truth of (75) involves two classes of men, the class of men who meet a nice woman and the class of men who meet a nice woman and smile at her: the sentence is true, roughly, if the cardinality of the second class is more than half that of the first. Note that the truth conditions do not involve the comparison of two sets of pairs of individuals - they do not compare the set of pairs $(a, b)$ such that $a$ is a man, $b$ a nice woman and $a$ meets $b$ with the set of pairs $(a, b)$ such that $a$ is a man, $b$ a nice woman, $a$ meets $b$ and $a$ smiles at $b$. One can see this by considering a situation in which one man meets lots of women and smiles at them all whereas the other men (say, there are 20 of them) meet very few women and never smile at any. With regard to such a situation intuition says that (75) is false, even though the pairs $(a, b)$ such that $a$ smiles at $b$ may be a clear majority within the set of pairs $(a, b)$ such that $a$ is a man, $b$ is a nice woman and $a$ meets $b$.

Thus, while the treatment of universal quantification in (74) creates the impression that the quantificational force resides somehow in the dynamic implication $\Rightarrow$, we cannot hope that this can be extended to non-standard quantifiers by working out special variants of dynamic implication. For suppose that we represent (75) as (76). 


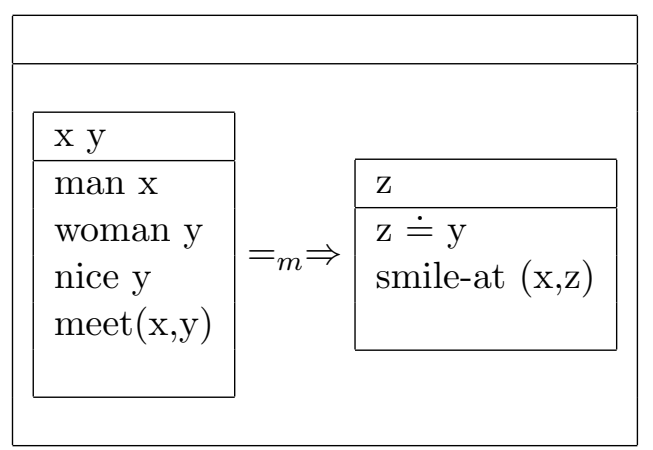

The semantics of $=_{m} \Rightarrow$ is given by:

- ${ }_{s} \llbracket D_{1}={ }_{m} \Rightarrow D_{2} \rrbracket_{s^{\prime}}^{\mathcal{M}}$ iff $s=s^{\prime}$ and for most assignments $s_{1}$ with ${ }_{s} \llbracket D_{1} \rrbracket_{s_{1}}^{\mathcal{M}}$ there is an assignment $s_{2}$ with $s_{1} \llbracket D_{2} \rrbracket_{s_{2}}^{\mathcal{M}}$.

Unfortunately, this analysis gives the wrong truth conditions. In the example case, it quantifies over man-woman pairs instead of individual men. This problem (called the proportion problem in the literature) suggests that generalised quantifiers be added explicitly to the representation language; see the Chapter on Quantification in this Handbook.

Assuming that what is true for most holds in essence also for every, the above considerations show that the roles which $x$ and $y$ play in (74) are not identical. The role played by $x$, the 'variable bound by the quantifier', is special in that it is $x$, and only $x$, which determines between which sets the generalised quantifier relation expressed by the determiner of the quantifying NP can be said to hold. A notation that singles out the variable of quantification achieves this. These considerations lead to the following Generalised Quantifier notation for (74) and (76).

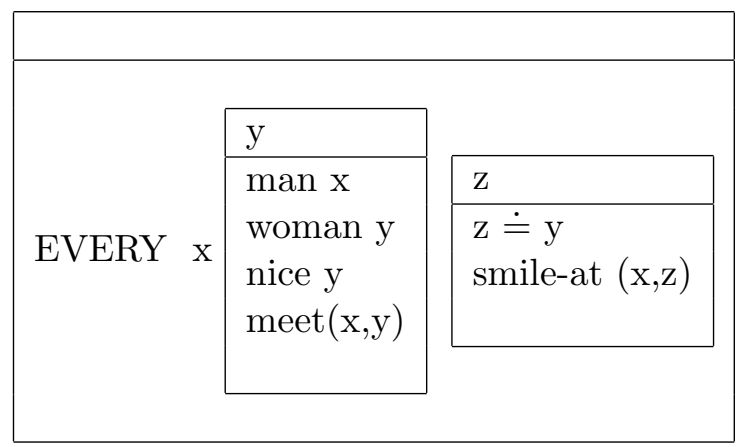




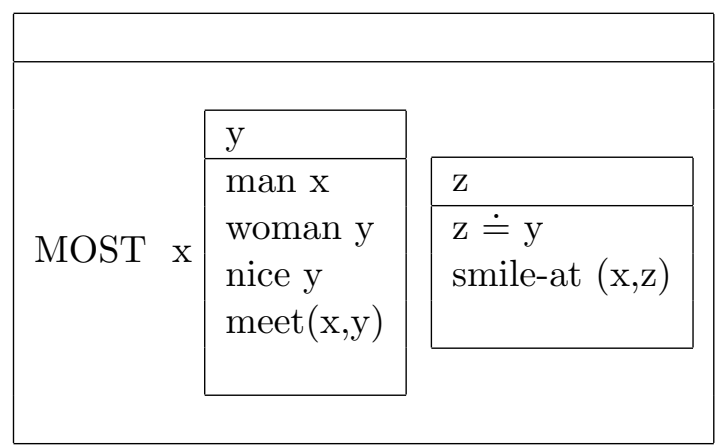

We can now revise the treatment of quantification in our fragment and extend the coverage to other non-standard quantifiers such as most, at most half, at least seven, as follows. Every is the function of type $K \rightarrow K \rightarrow t$ that takes two $K$ expressions $P$ and $Q$ and an input context $c$, checks whether all items satisfying

$$
\lambda x \mapsto \exists c^{\prime}|c|=i \wedge \operatorname{Pi} \hat{c} x c^{\prime}
$$

also satisfy

$$
\lambda x \mapsto \exists c^{\prime \prime} \exists c^{\prime}|c|=i \wedge P i \hat{c^{\wedge} x} c^{\prime \prime} \wedge P i \hat{c} x c^{\prime \prime} \wedge Q i c^{\prime \prime} c^{\prime},
$$

and if so, returns $c$ as output context (and otherwise fails). Similarly for the other generalized quantifiers.

Note that this interpretation also takes care of the 'internal dynamics' of the quantification. To spell this out in terms of box satisfaction conditions we use $s[x]$ for an assignment which differs at most from $s$ in the value assigned to $x$, and $\mathcal{M}, s \models D$ for truth in $\mathcal{M}$, given $s$.

(79) ${ }_{s} \llbracket Q x\left(D_{1}, D_{2}\right) \rrbracket_{s^{\prime}}^{\mathcal{M}}$ iff $s=s^{\prime}$ and the set of assignments $s[x]$ for which $\mathcal{M}, s[x]=D_{1}$ is Q-related to the set of assignments $s[x]$ for which $\mathcal{M}, s[x] \models D_{1} ; D_{2}$.

Note the fact that the meaning of $D_{1}$ figures both in the definition of the restriction set $R$ of the quantifier and in the definition of its body set $B$. The reason for this is that $D_{1}$ may introduce referents that have to be resolved in order to get at the meaning of the body set. In the example sentence we have to compare the set of men who meet a nice woman with the set of men who meet a nice woman at whom they smile. Saying that we want to 
compare the set of 'men who meet a nice woman' with that of 'men who smile at her' will not do, for the specification of the second set contains an unresolved pronominal reference.

It seems intuitively clear that the pronoun her is to be interpreted as anaphoric to the indefinite NP a woman. It is one of the central claims of DRT that this kind of anaphoric connection is possible because the material of the quantifying sentence that makes up the restrictor is also, implicitly, part of the quantifier's body. This principle also explains why natural language quantifiers are always conservative, i.e. express relations between sets with the property that for any sets $A$ and $B, A$ stands in the relation to $B$ iff it stands in the relation to $A \cap B$. They satisfy this equation because a natural language quantification with restrictor condition $P$ and body condition $Q$ has a logical form to the effect that the quantifier relation holds between the extension of $P$ and the extension of $P \wedge Q$. Conservativity is built directly into the logical form.

For the example sentence with most, (79) gives the following meaning: for most men who meet a nice woman it holds that they smile at at least one nice woman that they meet. This is called the weak reading of the dynamic generalised quantifier. Note that under the semantics given above, EVERY $x((y ; R x y), S x y)$ is not equivalent to $(x ; y ; R x y) \Rightarrow S x y$. In the first expression $y$ has existential force, in the second, $y$ has universal force. There is no perfect agreement among speakers whether (73) and (75) can be interpreted as having the weak reading. Some prefer the so-called strong reading:

(80) ${ }_{s} \llbracket Q x\left(D_{1}, D_{2}\right) \rrbracket_{s^{\prime}}^{\mathcal{M}}$ iff $s=s^{\prime}$ and the set of assignments $s[x]$ for which $\mathcal{M}, s[x]=D_{1}$ is Q-related to the set of assignments $s[x]$ for which $\mathcal{M}, s[x] \mid=\neg\left(D_{1} \bullet \neg D_{2}\right)$.

Under this interpretation for the quantifiers, EVERY $x((y ; R x y), S x y)$ and $(x ; y ; R x y) \Rightarrow S x y$ are equivalent.

In the definition of strong readings for the quantifiers, we again use the restriction set to resolve pronominal references in the specification of the body set, and again the conservativity property of the generalized quantifier denotation ensures that this does not change the truth conditions. In example case $(78)$ the strong reading can be paraphrased as: for most men who meet 
a nice woman it holds that they smile at all the nice women that they meet. See the Chapter on Quantification in this Handbook for more information on how to choose between weak and strong readings of dynamic quantifiers.

\section{Representing Tense and Aspect in Texts}

As was said in Section 3 above, discourse representation theory was motivated by a desire to give a systematic account of the interpretation of unbound nominal and temporal anaphora in context. In example (81), there is not only an intended anaphoric link between the indefinite subject of the first sentence and the pronominal subject of the second, but also between the tenses of the verbs in the two sentences.

(81) A man entered the White Hart. He smiled.

The events described in example (81) are naturally understood as sequential, with the event of entering preceding the event of smiling. Also, the past tense indicates that both events precede the time of speech. A plausible DRS representation for the example that makes this temporal anaphoric link explicit is given in (82).

\begin{tabular}{|l|}
\hline$u_{1} u_{2} u_{3} e_{1} e_{2}$ \\
\hline $\operatorname{man} u_{1}$ \\
$u_{2} \doteq W H$ \\
enter $\left(e_{1}, u_{1}, u_{2}\right)$ \\
$t\left(e_{1}\right)<n$ \\
$u_{3} \doteq u_{1}$ \\
smile $\left(e_{2}, u_{3}\right)$ \\
$t\left(e_{1}\right)<t\left(e_{2}\right)$ \\
$t\left(e_{2}\right)<n$ \\
\hline
\end{tabular}

In this representation we have given the verbs a Davidsonian event argument (Davidson [16]), and we have assumed that $t(e)$ denotes the temporal interval during which the event $e$ takes place. Also, we assume that $n$ ('now') refers to an interval during which the text is uttered (the speech interval).

As the example representation indicates, we assume an ontology of events, with temporal intervals at which these take place. Furthermore, we assume 
that the set of temporal intervals is ordered by precedence $<$ and by temporal inclusion $\sqsubseteq$. We assume that $t_{1}<t_{2}$ expresses that interval $t_{1}$ completely precedes $t_{2}$, i.e., the end of $t_{1}$ is before the beginning of $t_{2}$, while $t_{1} \sqsubseteq t_{2}$ expresses that the beginning of $t_{2}$ is not later than the beginning of $t_{1}$ and the end of $t_{2}$ is not earlier than the end of $t_{1}$.

It is plausible to further assume that $<$ is irreflexive and transitive, while $\sqsubseteq$ is a partial order (reflexive and transitive). Also, the following are plausible interaction principles:

monotonicity $(x \sqsubseteq y \wedge y<z \wedge u \sqsubseteq z) \rightarrow x<u$.

convexity $(x \sqsubseteq u \wedge x<y \wedge y<z \wedge z \sqsubseteq u) \rightarrow y \sqsubseteq u$.

But we will not dwell on the underlying temporal ontology; for further information on the temporal logic of intervals we refer to the Chapter on Temporality of this Handbook and to Van Benthem [8].

In (82) the smiling event $e_{2}$ is represented as following the entering event $e_{1}$. This is intuitively as it should be and has to do with the fact that in (81) the sentence reporting the smiling event comes after the one which reports the entering event. (Note that the interpretation given in (82) is not, or only barely, available when the sentences of (81) are reversed.) However, the order in which the sentences of a text appear is only one of several factors that determine the temporal relations between the events they mention. A second factor is aspect. For instance, when we replace the non-progressive smiled in (81) by the progressive was smiling, there is a strong tendency to understand the smiling as something that was going on while the man was entering the White Hart: the progressive of an activity verb like smile suggests, at least in narrative passages such as (81), simultaneity with the last mentioned event, rather than succession to it. Similarly, simultaneity rather than succession is suggested by a stative verb such as like. Consider example (83).

(83) A man ${ }^{1}$ entered the White Hart ${ }^{2}$. He 1 smiled. He 1 liked the place 2 .

In this example, the man's liking of the White Hart is not naturally interpreted as having been the case only after his smiling. Rather, it seems that the state of affairs of his liking the establishment obtained already as he was smiling, and possibly even before he came in. Thus, the representation of (83) should be as in (84): 


\begin{tabular}{|ll|}
\hline$u_{1} u_{2} u_{3} u_{4} u_{5} e_{1} e_{2} e_{3}$ & \\
\hline man $u_{1}$ & $u_{2} \doteq W H$ \\
enter $\left(e_{1}, u_{1}, u_{2}\right)$ & $t\left(e_{1}\right)<n$ \\
smile $\left(e_{2}, u_{3}\right)$ & $u_{3} \doteq u_{1}$ \\
& $t\left(e_{1}\right)<t\left(e_{2}\right)$ \\
& $t\left(e_{2}\right)<n$ \\
& $u_{4} \doteq u_{1}$ \\
place $\left(u_{5}\right)$ & $u_{5} \doteq u_{2}$ \\
like $\left(e_{3}, u_{4}, u_{5}\right)$ & $t\left(e_{2}\right) \sqsubseteq t\left(e_{3}\right)$ \\
& $t\left(e_{3}\right)<n$ \\
\hline
\end{tabular}

When we consider the question whether one should assume that the man's liking the place in (83) anteceded his entering the White Hart, we perceive a further factor that is important for the interpretation of temporal relations. In order that a text is perceived as coherent, its successive sentences must be seen as standing in certain rhetorical relations to each other. (Halliday \& Hasan [34], Mann \& Thompson [48]). One such relation is explanation, a relation which holds between two neighbouring sentences (or sometimes larger units, consisting of several sentences) when the later sentence or sentence group provides an explanation for what is claimed by the earlier sentence or group. Like many other rhetorical relations explanation carries certain implications form temporal order. For instance, when, say, two sentences $S$ and $S^{\prime}$ are interpreted as standing in the explanation relation, with $S^{\prime}$ providing an explanation for what is said in $S$, the event or state described by $S^{\prime}$ cannot be later than that described in $S$. We see this when we look closely at (83): the man's liking the place can either be taken as an explanation of his smiling or as an explanation of why the man went to the White Hart in the first place. The first interpretation entails that his liking the place did not start after his smiling, but it leaves open whether he liked the place only upon entering it or already before. According to the second interpretation the man must have liked the place even before he went in.

We have dwelt on this dimension of the interpretation of the temporal relations in (83) to indicate how complicated the matter of interpreting temporal relations is and how much it depends on pragmatic factors such as discourse coherence and rhetorical relations. Just as with pronominal anaphora, linguistic form does in general no more than impose a frame of constraints within which the precise interpretation of temporal relations must be decided on other grounds. 
For a presentation of the semantics of temporal reference within the very limited space available here this poses a dilemma. On the one hand, a presentation that does justice to what is now known about the interactions between the different factors mentioned above is out of the question. On the other, a general treatment of the purely grammatical constraints on temporal reference would, in view of its inevitable lack of specificity, be rather uninformative. We have therefore chosen to concentrate on a certain small subclass of texts, in which rhetorical relations are fully determined by linguistic form (by the order of the sentences in the text, by the tenses of the verbs and by their aspectual properties). (81) and (83) are both instances of this class.

The central idea behind the treatment we will present goes back to Reichenbach [54]. The interpretation of the tenses involves relating the event or state described to a reference point. For instance, for unembedded cases of the simple past tense, the reference point is provided by the context in which the given past tense sentence occurs. In texts of the kind to which our theory is intended to apply it is the immediately preceding sentence which supplies the reference point. How the reference point is used to temporally locate the event or state described by the sentence in question depends on whether the sentence has stative or non-stative aspect (or, what comes to the same in our terminology, whether what the sentence describes is a state or an event). For past tense sentences, the difference that aspect makes is illustrated by the distinct interpretations that are assigned to the second and the third sentence of (83) - the event described by the second sentence is interpreted as following the reference point by the preceding sentence, the state described by the third sentence as obtaining at the reference point provided by its predecessor. Moreover, an event sentence like the second sentence of (83) resets the reference point it inherits from the context to the event it itself introduces, whereas a stative sentence like the third one passes the reference point on to the next sentence unchanged. (To test this, see what happens when one adds a fourth sentence, stative or non-stative, on to $(83))$.

Besides playing a role in locating the described event or state in relation to the reference point, tense forms usually also have an 'absolute' semantic impact in that they relate the described state or event to the utterance time. For instance, unembedded occurrences of the past tense imply that the state or event lies before the utterance time and unembedded occurrences of the English present tense imply, with few exceptions, location at the utterance 
time.

For the limited domain to which our 'mini theory' is meant to apply, the use and modification of reference points can be elegantly handled along the lines proposed by Muskens [49]. As noted there, in a dynamic set-up it is natural to implement the reference interval as a register $r$ to which a new value get assigned for a non-stative verb, while the value is unaffected for stative verbs. For instance, the lexical entry for smiled specifies that the interval of the smiling event is constrained to follow the current reference interval, that the reference interval is reset to the interval of the event, and that the event interval has to precede the interval of speech:

$$
\lambda v(e ; \text { smile }(e, v) ; r<t(e) ; r:=t(e) ; r<n) .
$$

Here $r:=t(e)$ is shorthand for $r ; r \doteq t(e)$.

For verbs denoting stative events, the representation is the same, except for the fact that now the current reference interval has to be included in the event interval, and the reference interval is not reset. Here is a lexical entry for liked:

$$
\lambda \mathcal{P} \lambda u(\mathcal{P} \lambda v(e ; \text { like }(e, u, v) ; r \sqsubseteq t(e) ; r<n)) .
$$

Figure 10 gives a list of lexical entries for stative and non-stative main verbs and for temporal auxiliary verbs.

\begin{tabular}{|l|l|l|l|}
\hline expression & category & translates to & type \\
\hline does not & AUX & $\lambda P \lambda v \neg(P(v) ; r \sqsubseteq n)$ & $((\mathrm{e}, \mathrm{T}),(\mathrm{e}, \mathrm{T}))$ \\
did not & AUX & $\lambda P \lambda v \neg(P(v) ; r<n)$ & $((\mathrm{e}, \mathrm{T}),(\mathrm{e}, \mathrm{T}))$ \\
will & AUX & $\lambda P \lambda v(P(v) ; n<r)$ & $((\mathrm{e}, \mathrm{T}),(\mathrm{e}, \mathrm{T}))$ \\
will not & AUX & $\lambda P \lambda v \neg(P(v) ; n<r)$ & $((\mathrm{e}, \mathrm{T}),(\mathrm{e}, \mathrm{T}))$ \\
smiles & $\mathrm{VP}($ Tensed $)$ & $\lambda v(e ;$ smile $(e, v) ; r<t(e) ; r:=t(e) ; r \sqsubseteq n)$ & $(\mathrm{e}, \mathrm{T})$ \\
smiled & VP(Tensed) & $\lambda v(e ;$ smile $(e, v) ; r<t(e) ; r: t(e) ; r<n)$ & $(\mathrm{e}, \mathrm{T})$ \\
smile & VP(Inf) & $\lambda v(e ;$ smile $(e, v) ; r<t(e) ; r:=t(e))$ & $(\mathrm{e}, \mathrm{T})$ \\
hates & TV(Tensed) & $\lambda \mathcal{P} \lambda u(\mathcal{P} \lambda v(e ; h a t e(e, u, v) ; r \sqsubseteq t(e) ; r \sqsubseteq n))$ & $(((\mathrm{e}, \mathrm{T}), \mathrm{T}),(\mathrm{e}, \mathrm{T}))$ \\
hated & TV(Tensed) & $\lambda \mathcal{P} \lambda u(\mathcal{P} \lambda v(e ;$ hate $(e, u, v) ; r \sqsubseteq t(e) ; r<n))$ & $(((\mathrm{e}, \mathrm{T}), \mathrm{T}),(\mathrm{e}, \mathrm{T}))$ \\
hate & TV(Inf) & $\lambda \mathcal{P} \lambda u(\mathcal{P} \lambda v(e ; h a t e(e, u, v) ; r \sqsubseteq t(e))$ & $((\mathrm{e}, \mathrm{T}), \mathrm{T}),(\mathrm{e}, \mathrm{T}))$ \\
likes & TV(Tensed) & $\lambda \mathcal{P} \lambda u(\mathcal{P} \lambda v(e ;$ like $(e, u, v) ; r \sqsubseteq t(e) ; r \sqsubseteq n))$ & $(((\mathrm{e}, \mathrm{T}), \mathrm{T}),(\mathrm{e}, \mathrm{T}))$ \\
liked & TV(Tensed) $)$ & $\lambda \mathcal{P} \lambda u(\mathcal{P} \lambda v(e ;$ like $(e, u, v) ; r \sqsubseteq t(e) ; r<n))$ & $(((\mathrm{e}, \mathrm{T}), \mathrm{T}),(\mathrm{e}, \mathrm{T}))$ \\
like & TV(Inf) & $\lambda \mathcal{P} \lambda u(\mathcal{P} \lambda v(e ; l i k e(e, u, v) ; r \sqsubseteq t(e) ;))$ & $(((\mathrm{e}, \mathrm{T}), \mathrm{T}),(\mathrm{e}, \mathrm{T}))$ \\
\hline
\end{tabular}

Figure 10: Lexical entries for main and auxiliary verbs.

Note that in defining disjoint merge for fragments involving the markers $r$ and $n$ for the reference and the speech interval, we have to make sure that 
these never get renamed. For $n$, we get this for free, for an inspection of the lexical entries makes clear that $n$ is a fixed marker of every DRS, as it never gets introduced. For $r$ matters are different: $r:=t(e)$ is shorthand for $r ; r \doteq t(e)$, so $r$ does get introduced. But we do not want $r:=t\left(e_{1}\right) ; D_{1} \bullet r:=$ $t\left(e_{2}\right) ; D_{2}$ to reduce to $r:=t\left(e_{1}\right) ; D ; r^{\prime}:=t\left(e_{2}\right) ;\left[r^{\prime} / r\right] D_{2}$. To ensure that this does not happen, it is enough to exclude $r$ from the set of reference markers; this guarantees that $r:=t\left(e_{1}\right) ; D_{1} ; r:=t\left(e_{2}\right) ; D_{2}$ is a proper DRS if $D_{1} ; D_{2}$ is one, because $r \notin \operatorname{intro}\left(r:=t\left(e_{2}\right) ; D_{2}\right)$.

Let us go through the procedure of building the representation for (83), assuming the antecedent and anaphoric indices to be as given in the example. The representation of entered the White Hart becomes (85).

$$
\begin{aligned}
& \lambda \mathcal{P} \lambda u(\mathcal{P} \lambda v(e ; \text { enter }(e, u, v) ; r<t(e) ; r:=t(e) ; r<n)) \\
& \left(\lambda P\left(u_{2} \doteq W H \bullet P\left(u_{2}\right)\right)\right) .
\end{aligned}
$$

After $\beta$ reduction:

$$
\lambda u\left(u_{2} \doteq W H \bullet\left(e ; \text { enter }\left(e, u, u_{2}\right) ; r<t(e) ; r:=t(e) ; r<n\right)\right) .
$$

Combining with the translation of a man and reducing the result gives (87).

$$
u_{1} \bullet \operatorname{man} u_{1} \bullet\left(u_{2} \doteq W H \bullet\left(e ; \text { enter }\left(e, u_{1}, u_{2}\right) ; r<t(e) ; r:=t(e) ; r<n\right)\right)
$$

Merge reduction with the identical renaming gives:

$$
u_{1} ; \text { man } u_{1} ; u_{2} \doteq W H ; e ; \text { enter }\left(e, u_{1}, u_{2}\right) ; r<t(e) ; r:=t(e) ; r<n \text {. }
$$

Similarly, we get for he smiled, after $\beta$ and merge reduction:

$$
e ; \operatorname{smile}\left(e, u_{1}\right) ; r<t(e) ; r:=t(e) ; r<n \text {. }
$$

The text consisting of the first two sentences gets the following translation after $\beta$ reduction:

$$
\begin{aligned}
& u_{1} ; \text { man } u_{1} ; u_{2} \doteq W H ; e ; \text { enter }\left(e, u_{1}, u_{2}\right) ; r<t(e) ; r:=t(e) ; r<n \\
& \text { - } e ; \text { smile }\left(e, u_{1}\right) ; r<t(e) ; r:=t(e) ; r<n .
\end{aligned}
$$

After merge reduction, this becomes: 


$$
\begin{aligned}
& u_{1} ; \text { man } u_{1} ; u_{2} \doteq W H ; e ; \text { enter }\left(e, u_{1}, u_{2}\right) ; r<t(e) ; r:=t(e) ; r<n ; \\
& e_{2} ; \operatorname{smile}\left(e_{2}, u_{1}\right) ; r<t\left(e_{2}\right) ; r:=t\left(e_{2}\right) ; r<n .
\end{aligned}
$$

The translation of the third sentence from the discourse, after $\beta$ and merge reduction:

$$
u_{3} ; u_{3} \doteq u_{2} ; \text { place } u_{3} ; e ; \text { like }\left(e, u_{1}, u_{3}\right) ; r \sqsubseteq t(e) ; r<n \text {. }
$$

The translation of the whole example, after $\beta$ and merge reduction:

$$
\begin{aligned}
& u_{1} ; \text { man } u_{1} ; u_{2} \doteq W H ; e ; \text { enter }\left(e, u_{1}, u_{2}\right) ; r<t(e) ; r:=t(e) ; r<n ; \\
& e_{2} ; \text { smile }\left(e_{2}, u_{1}\right) ; r<t\left(e_{2}\right) ; r:=t\left(e_{2}\right) ; r<n ; \\
& u_{3} ; u_{3} \doteq u_{2} ; \text { place } u_{3} ; e_{3} ; \text { like }\left(e_{3}, u_{1}, u_{3}\right) ; r \sqsubseteq t\left(e_{3}\right) ; r<n .
\end{aligned}
$$

Evidently this treatment of temporal reference is to be seen as no more than a hint of the direction that a fully fledged account of tense and aspect for a language like English might take. One feature of our treatment that ought to be changed is the use of separate lexical entries for full forms of verbs, such as smiled and smiles. What one would like to have instead is specifications of the meaning and/or function of the different tenses, such that when these are applied to the entries for the infinitival forms of our mini-lexicon we get the entries of the corresponding full forms as results. For instance, one might consider assigning the Simple Past the following entry

\begin{tabular}{|l|l|l|l|}
\hline expression & category & translates to & type \\
\hline Simple Past & $\mathrm{VP}($ Tensed $) / \mathrm{VP}($ Perf $)$ & $\lambda P \lambda v(P(v) ; r<n)$ & $((\mathrm{e}, \mathrm{T}),(\mathrm{e}, \mathrm{T}))$ \\
\hline
\end{tabular}

Indeed, applying this entry to the entries for smile and like produces the translations that our lexicon specifies for smiled and liked.

But here it behoves to repeat an earlier caveat. Tense forms do not always function in the same way. In particular, embedded occurrences of tenses often behave quite differently than when they occur in unembedded positions. (To cite just one example, involving the simple past, recall Baker's: "I thought you were going to say that you had only one trick to play." Here the past tense of had is compatible with the event in question being located in the future of the utterance time.) So, if we adopt the entry just proposed as entry for the 'Past Tense' in general, we will have to distinguish carefully 
between occurrences of the Past Tense in the semantic sense characterized by this entry on the one hand and, on the other hand, arbitrary occurrences of simple past tense morphology. But this is a distinction which requires a careful revision of the syntax-semantics interface used in our mini-fragment; and it is only one example among many which render such a revision necessary.

Another matter which seriously complicates the treatment of temporal reference is aspect. We already saw that the temporal relations between the states and events that are mentioned by sentences in a text depend in part on the aspectual properties of those sentences (i.e., in our terminology, on whether what they describe is a state or an event) and that the aspectual properties of those sentences depend in their turn on the aspectual properties of the verbs they contain. However, as noted explicitly first in Verkuyl [66], the aspectual properties of a sentence depend not just on its verb but on several other factors as well. Prominent among those factors is the question whether the verb has been modified by some aspectual operator, such as the English perfect or progressive, or aspectual control verbs such as begin, stop or go on. It is natural to try and treat aspectual modifiers along the same lines as we have suggested for the tenses, viz by assigning them their own lexical entries, which then should combine systematically with the entry of any verb to which the operators can be applied (e.g. through functional application of the operator entry to the verb entry). But here we encounter a new difficulty, which is especially noticeable in relation to the progressive, and known in that context as the imperfective paradox. A simple-minded analysis of the progressive might treat it as transforming a given verb phrase VP into one which describes a process or state holding at precisely those times that fall within the duration of any state or event described by VP. With telic verbal predicates such as cross the street, however, this analysis breaks down, for a sentence involving the progressive of such a verb phrase can be true at times when an event described by the embedded VP did not actually happen. For instance, The old lady was crossing the street may be true with respect to times not included in the duration of any crossingthe-street event. For the lady may have changed her mind when she got halfway and turned around to the sidewalk from which she started, or she may have become a victim to the incalculable brutalities of motorized traffic. Thus the semantic relation between progressives and their underlying VPs is in general an intensional rather than a purely extensional one, and a fully satisfactory analysis of this intensional relationship is still lacking. 
Formulating an entry for the English perfect, which transforms a verb phrase VP into one which describes result states of events or states described by VP, may at first seem less problematic: the states described by the application of the perfect hold at precisely those times which follow a state or event of the type defined by the operand. But when one looks at the semantics of the perfect more closely, such simplicity proves illusory. It is part of the meanings of many perfects that the event of which the described state is understood to be the result did not just happen at some earlier time or other, but that it happened only recently, or that its influence is still tangible at the time of the result state; and these additional meaning components cannot be analyzed in purely extensional terms any more than the relationship between progressive and non-progressive uses of telic verb phrases.

For the perfect it is nevertheless possible to finesse the intensionality problem by assuming a relation $\leadsto$ between events and states which holds between $e$ and $e^{\prime}$ when $e^{\prime}$ is the result state of $e$. We adopt the obvious assumption that $e \leadsto e^{\prime}$ entails $t(e)<t\left(e^{\prime}\right)$. Using $\leadsto,(94)$ might be represented as (95).

(94) Bill has smiled.

$$
\begin{array}{|l|}
\hline e_{1} e_{2} \\
\hline u \doteq b \\
\text { smile }\left(e_{1}, u\right) \\
e_{1} \leadsto e_{2} \\
t\left(e_{2}\right) \sqsubseteq n
\end{array}
$$

This does not yet constrain the effect on the wider context. The effect is roughly this. First the current value of the reference interval is saved. Then $r$ is reset to a value earlier than its old value. Next the verb is evaluated with respect to the shifted reference interval. Then the old value is restored, and finally the reference interval is located with respect to the speech interval (Muskens [49]).

Using $o$ as a store for the old value of $r$, we get the following DRS that also takes the external effects into account: 


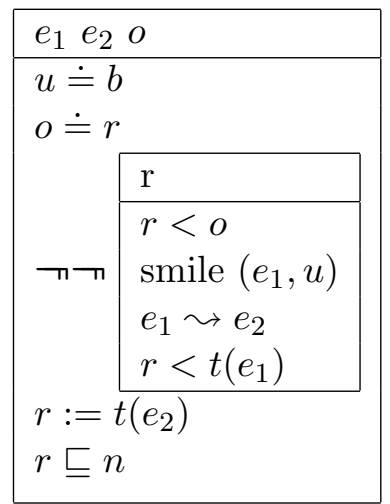

For a compositional account, we have to assume that we can get access to the event parameter of a verb, so a typical entry for untensed verbs will now look like this:

\begin{tabular}{|l|l|l|l|}
\hline expression & category & translates to & type \\
\hline smile & $\mathrm{VP}(\operatorname{Inf})$ & $\lambda e \lambda v($ smile $(e, v) ; r<t(e) ; r:=t(e))$ & $(\mathrm{e},(\mathrm{e}, \mathrm{T}))$ \\
\hline
\end{tabular}

The entry of the perfective operator introduces two events: the verb phrase event and the consequent state (assume $\mathcal{R}$ ranges over type $(e,(e, T))$ ).

\begin{tabular}{|l|l|l|c|}
\hline expression & category & translates to & type \\
\hline PERF & $\mathrm{VP}($ Perf $) /$ & $\lambda \mathcal{R} \lambda v\left(e_{1} ; e_{2} ; o:=r ;\right.$ \\
& $\mathrm{VP}($ Inf $)$ & $\begin{array}{l}\boldsymbol{\neg}\left(r ; r<o ; \mathcal{R}\left(e_{1}\right)(v) ; e_{1} \sim e_{2}\right) ; \\
\left.r<t\left(e_{2}\right) ; r:=t\left(e_{2}\right)\right)\end{array}$ & $((\mathrm{e},(\mathrm{e}, \mathrm{T}))$, \\
& & $(\mathrm{e}, \mathrm{T}))$ \\
& & \\
\hline
\end{tabular}

Temporal auxiliaries will now have the effect of putting further temporal constraints, as discussed above. For instance the present tense form has of the perfect auxiliary have could be given the following entry:

\begin{tabular}{|l|l|l|l|}
\hline expression & category & translates to & type \\
\hline has & $\mathrm{VP}($ Tensed $) / \mathrm{VP}($ Perf $)$ & $\lambda P \lambda v(P(v) ; r \sqsubseteq n)$ & $((\mathrm{e}, \mathrm{T}),(\mathrm{e}, \mathrm{T}))$ \\
\hline
\end{tabular}

This section has presented a catalogue of problems rather than a list of fully satisfactory solutions. The emphasis on problems with the analysis of tense and aspect may have served to illustrate a dilemma that one faces in formal approaches to the semantics of natural language discourse such as 
DRT. The dilemma is this: the more closely one tries to stick to the ideal of strict compositionality when dealing with the manifold complexities of the syntax-semantics interface of natural languages, the trickier the analysis tends to become, especially if discourse effects are to be taken into account too.

There exists a good deal of work within DRT, current as well as past, which has been prepared to sacrifice certain aspects of this ideal in pursuit of a more flexible architecture that can be fitted more easily to the requirements that certain linguistic phenomena seem to impose. This does not mean that this work ignores the fundamental compositional imperative of explaining how grammars can be finitely encoded and languages can be used by beings whose knowledge of language takes this finitary form. In particular, a good part of the work within DRT on the problems of tense and aspect has opted for such a relaxation of strict compositionality. However, experience of the past ten years has shown that often, once the phenomena have been properly understood and have been given a systematic description using means that are not strictly compositional, it is then possible to also find a way of accounting for those phenomena that is strictly compositional, as well as attractive in other ways. Whether attractive strictly compositional solutions will become available in all cases is yet to be seen.

\section{Extensions and Variations}

An important extension of the representation language concerns the singular/plural distinction. Singular and plural reference markers should be distinguished, and a constraint imposed that singular pronouns are linked to singular discourse referents, plural pronouns to plural reference markers. Accounting for plural anaphoric possibilities along these lines involves quite a lot of further work, however, as delicate issues concerning the formation of plurals by means of summation and abstraction, and the interpretation of dependent plurals have to be dealt with (Kamp and Reyle [38], Chapter 4).

Another fruitful application area for theories about the representation of discourse in context is the area of presupposition. Presuppositions can get cancelled or weakened by an evolving context; in other words, presupposition projection is a dynamic phenomenon. Approaches to presupposition in connection with discourse representation are of two kinds. The first kind 
exploits the representationalism inherent in the framework. See, e.g., Van der Sandt [59], where the presupposition facts get accounted for in terms of manipulations of the representations. The second kind does not assume representationalism but exploits the dynamic aspect of the theory by providing a partial dynamic semantics fitting the presupposition facts. See, e.g., the account of the presuppositions of definite descriptions in Van Eijck [20], which does not depend on properties of the representations, but only on the underlying 'error state' semantics. Further references in the Chapter on Presupposition in this Handbook.

A next extension concerns the representation of belief sentences. The Hob Nob sentence from Section 3 provides an example of a belief puzzle that seems amenable to solution within the present framework. A theory of representation of discourse in context holds a particular promise for the treatment of belief because the representation structures themselves could be viewed as a kind of mental representation language; thus a belief relation could typically be modelled as a relation between a subject and a representation structure (Asher [3]).

The plausibility of using Discourse Representation Structures to model belief and other propositional attitudes is closely connected with the existence of cognitively plausible inference systems for DRSs. For work on proof theories for DRSs see Sedogbo and Eytan [61], Saurer [60] and Kamp and Reyle [39].

A different approach is reasoning about discourse structures with assertion logic and dynamic logic. Assume a language of quantified dynamic logic with discourse representation structures as program modalities $\langle\boldsymbol{D}\rangle$ and $[\boldsymbol{D}]$. Then $\langle\boldsymbol{D}\rangle \phi$ and $[\boldsymbol{D}] \phi$ get interpreted as follows:

- $\mathcal{M}, s \models\langle\boldsymbol{D}\rangle \phi$ iff there is an $s^{\prime}$ with ${ }_{s} \llbracket D \rrbracket_{s^{\prime}}^{\mathcal{M}}$ and $\mathcal{M}, s^{\prime}=\phi$.

- $\mathcal{M}, s \models[\boldsymbol{D}] \phi$ iff for all $s^{\prime}$ with ${ }_{s} \llbracket D \rrbracket_{s^{\prime}}^{\mathcal{M}}$ it holds that $\mathcal{M}, s^{\prime}=\phi$.

An axiomatisation of discourse representation theory along the same lines as the calculus for dynamic predicate logic [33] given in Van Eijck [21] is now readily available. Some example principles of this calculus are:

$$
\begin{aligned}
&\langle\neg D\rangle \phi([\boldsymbol{D}] \perp \wedge \phi) . \\
&\left\langle\boldsymbol{D}_{\mathbf{1}} \Rightarrow \boldsymbol{D}_{\mathbf{2}}\right\rangle \phi \leftrightarrow\left(\left[\boldsymbol{D}_{\mathbf{1}}\right]\left\langle\boldsymbol{D}_{\mathbf{2}}\right\rangle \top \wedge \phi\right) . \\
&\left\langle\boldsymbol{D}_{\mathbf{1}} ; \boldsymbol{D}_{\mathbf{2}}\right\rangle \phi \leftrightarrow\left\langle\boldsymbol{D}_{\mathbf{1}}\right\rangle\left\langle\boldsymbol{D}_{\mathbf{2}}\right\rangle \phi .
\end{aligned}
$$


For marker introduction we have:

$$
\langle\boldsymbol{u}\rangle \phi \leftrightarrow \exists u \phi,
$$

or dually:

$$
[\boldsymbol{u}] \phi \leftrightarrow \forall u \phi
$$

For atoms we have:

$$
\left\langle\boldsymbol{P t _ { 1 }} \cdots \boldsymbol{t}_{\boldsymbol{n}}\right\rangle \phi \leftrightarrow\left(P t_{1} \cdots t_{n} \wedge \phi\right),
$$

or dually:

$$
\left[\boldsymbol{P t _ { 1 }} \cdots \boldsymbol{t}_{\boldsymbol{n}}\right] \phi \leftrightarrow\left(P t_{1} \cdots t_{n} \rightarrow \phi\right) .
$$

The calculus nicely demonstrates the way in which discourse representation theory gives universal force to the markers introduced in the antecedent of an if-then clause.

(97) If a man greets a woman he smiles at her.

(98) $(x ; M x ; y ; W y ; G x y) \Rightarrow S x y$.

The truth conditions of (97), represented as (98), are given by the following calculation that uses the principles above.

$$
\begin{aligned}
& \langle(\boldsymbol{x} ; \boldsymbol{M x} ; \boldsymbol{y} ; \boldsymbol{W} \boldsymbol{y} ; \boldsymbol{G x} \boldsymbol{y}) \Rightarrow \boldsymbol{S} \boldsymbol{x} \boldsymbol{y}\rangle \top \\
& \leftrightarrow[\boldsymbol{x} ; \boldsymbol{M x} ; \boldsymbol{y} ; \boldsymbol{W} \boldsymbol{y} ; \boldsymbol{G} \boldsymbol{x y}]\langle\boldsymbol{S} \boldsymbol{x} \boldsymbol{y}\rangle \top \\
& \leftrightarrow[\boldsymbol{x}][\boldsymbol{M x}][\boldsymbol{y}][\boldsymbol{W} \boldsymbol{y}][\boldsymbol{G x} \boldsymbol{y}]\langle\boldsymbol{S x y}\rangle \top \\
& \leftrightarrow \forall x([\boldsymbol{M x}][\boldsymbol{y}][\boldsymbol{W} \boldsymbol{y}][\boldsymbol{G x} \boldsymbol{y}]\langle\boldsymbol{S} \boldsymbol{x} \boldsymbol{y}\rangle \top) \\
& \leftrightarrow \cdots \\
& \leftrightarrow \forall x(M x \rightarrow \forall y(W y \rightarrow(G x y \rightarrow S x y))) .
\end{aligned}
$$

An important new direction is the theory of Underspecified Discourse Representation Structures which allows for representations that leave certain matters, such as scope relations between quantifiers and other operators, the distinction between distributive and collective readings of plural NPs, that between different readings of a given lexical item, etc. undecided. This work is of particular interest insofar as it has succeeded in developing proof theories that operate directly on the underspecified representations themselves (Reyle [55, 56]). 


\section{A Simplified representation of contexts}

As an extension of our treatment above we will now consider a simplified representation of contexts as stacks of references to entities.

If the context has name $c$ and length $i$, then the reference markers are called $c_{0}, \ldots, c_{i-1}$ (or $c[0], \ldots, c[i-1]$, for those who prefer the programmers' way of referring to array indexing). Extending a context $c$ of length $i$ with a 'fresh' reference marker can now consist of incrementing the length of the context to $i+1$ and adding marker $c_{i}$ to it. As we will show in the next section, this approach allows the formulation of an elegant type theoretical version of discourse representation theory, thus facilitating the definition of natural language fragments with a dynamic flavour in the manner of Montague grammar.

Representing discourse in context by means of context updating while putting appropriate constraints on the evolving context can be viewed as constructing a function with the following shape:

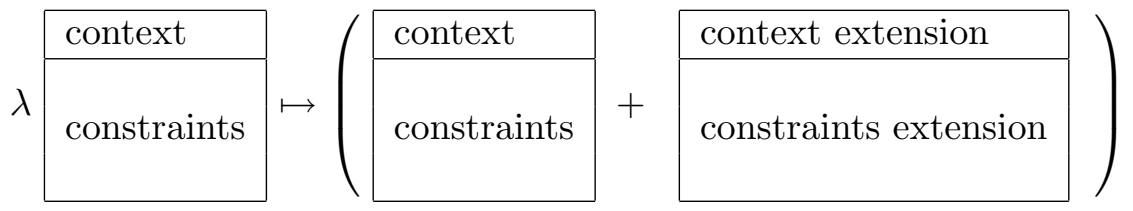

The compositional version of DRT presented in the fragment given in Section 10 below works according to this pattern. It assumes that contexts are lists of reference markers, together with their values, and that constraints are expressed as DRS conditions.

A simplified representation is possible by taking contexts to be lists of reference markers with canonical names $c_{0}, c_{1}, \ldots$ If we view a context as the one-variable version of Vermeulen's sequence semantics [67], then a context is just a stack of items:

\begin{tabular}{|l|l|l|l|l|l|}
\hline$c_{0}$ & $c_{1}$ & $c_{2}$ & $c_{3}$ & $c_{4}$ & $\cdots$ \\
\hline
\end{tabular}

Existential quantification now is context extension: it pushes a new item $d$ on the context stack:

\begin{tabular}{|l|l|l|l|}
\hline$c_{0}$ & $c_{1}$ & $c_{2}$ & $c_{3}$ \\
\hline
\end{tabular}


We can use indices to refer to the items:

\begin{tabular}{|c|c|c|c|c|l|c|c|}
\hline 0 & 1 & 2 & 3 & 4 & $\cdots$ & $n-1$ & $n$ \\
\hline$c_{0}$ & $c_{1}$ & $c_{2}$ & $c_{3}$ & $c_{4}$ & $\cdots$ & $c_{n-1}$ & $d$ \\
\hline
\end{tabular}

If $c$ is a context, $c[0]$ is its first element, and $|c|$ is its length. So the context elements are $c[0]$ up to $c[k]$ where $k=|c|-1$.

A context has the type of a list of entities. Call this type $[e]$. Assume $c, c^{\prime}$ are of type $[e]$ and that $x$ is of type $e$. Then we use $\hat{c} x$ to denote the result of extending context $c$ with item $x$. Note that the type of $\left(^{\wedge}\right)$ is $[e] \rightarrow e \rightarrow[e]$, and that $\hat{c^{\wedge} x}$ is a new context with $\left|\hat{c^{\wedge} x}\right|=|c|+1$ (the context length has increased by 1). Now we can define dynamic existential quantification as follows:

$$
\exists:=\lambda c \lambda c^{\prime} \mapsto \exists x\left(\hat{\left.c^{\wedge} x=c^{\prime}\right)}\right.
$$

Thus, the quantifier $\exists$ is interpreted as a context extender. It extends an input context $c$ with an element $x$ from the domain, and creates an output context $\hat{c} x$. More precisely, $\exists x\left(\hat{c} x=c^{\prime}\right)$ states the conditions under which $c^{\prime}$ is an appropriate output context, given that $c$ is the input context.

We see that $\exists$ has the type $[e] \rightarrow[e] \rightarrow t$. The operation $\exists$ takes a list of entities $c$ (an input context) and a second context $c^{\prime}$ (an output context), and it states the conditions under which $c$ and $c^{\prime}$ are properly related. In DRT terms, $\exists$ expresses 'take a new reference marker and extend the context with that marker'.

The type $[e] \rightarrow[e] \rightarrow t$ is essentially the type of a binary relation on contexts; call such relations on contexts context transitions. In a compositional account of context shifting, this is the fundamental type. A DRT version of extensional Montague grammar can now be viewed as a lift of the type $t$ to $[e] \rightarrow[e] \rightarrow t$.

Instead of conjunction (type $t \rightarrow t \rightarrow t$ ) we get sequential composition of contexts, with type $([e] \rightarrow[e] \rightarrow t) \rightarrow([e] \rightarrow[e] \rightarrow t) \rightarrow[e] \rightarrow[e] \rightarrow t)$. Assume that $\phi, \psi$ are context transitions (i.e., $\phi, \psi$ have type $[e] \rightarrow[e] \rightarrow t$ ) and that $c, c^{\prime}$ are contexts $\left(c, c^{\prime}\right.$ have type $\left.[e]\right)$. Then the following operation defines context composition:

$$
\phi ; \psi:=\lambda c \lambda c^{\prime} \mapsto \exists c^{\prime \prime}\left(\phi c c^{\prime \prime} \wedge \psi c^{\prime \prime} c^{\prime}\right)
$$

Since $\phi, \psi$ are parameters, we can define the operation ; as:

$$
\lambda \phi \lambda \psi \lambda c \lambda c^{\prime} \mapsto \exists c^{\prime \prime}\left(\phi c c^{\prime \prime} \wedge \psi c^{\prime \prime} c^{\prime}\right)
$$


The definitions of $\exists$ and ; are the key ingredients in the definition of the semantics of the indefinite determiner. Note ; defines a sequential merge operation for context transitions. Before we proceed, we introduce the type theoretic version of the introduction of a reference marker, by means of the following combinator $u$.

$$
u=\lambda i \lambda c \lambda c^{\prime} \mapsto|c|=i \wedge \exists x\left(\hat{\left.c^{\wedge} x=c^{\prime}\right) .}\right.
$$

Variable $i$ ranges over natural numbers, so this defines a function of type $\mathbb{N} \rightarrow[e] \rightarrow[e] \rightarrow t$. Writing the application $u i$ as $u_{i}$, we get from the definition of $u$ :

$$
u_{i}=\lambda c \lambda c^{\prime} \mapsto|c|=i \wedge \exists x\left(\hat{\left.c^{\wedge} x=c^{\prime}\right) .}\right.
$$

This means that $u_{i}$ denotes the context transition that consists of selecting an input context of length $i$ and extending that context with a new element.

The entry for the indefinite noun phrase $a$ man should express that an input context $c$ can be extended with a new element, that this new element has to be a man, that it also has to satisfy the body of the determiner, and that it will remain accessible for future reference. If we assume the context has length $i$, then the new element will be at position $i$ in the new context. This gives:

$$
\text { a man: } \quad \lambda Q \lambda c \mapsto \frac{c_{i}}{\text { Man } c_{i}} \quad ; Q i \quad \text { where } i=|c|
$$

But now we have to realize that the box notation is shorthand for a context transition. The fine structure is given by the sequential composition of $u_{i}$ (the operation for selecting a context of length $i$ and extending that context with a new element), and a context transition $M$ that consists of predicating the property Man of the new element in context.

The predication $Q$ has to be of a type that combines with an index $i$ to yield a context transition. This is type $\mathbb{N} \rightarrow[e] \rightarrow[e] \rightarrow t$, the type of natural number pointers into context transitions. We still have to abstract over the restriction of the indefinite. Let the type of the restriction be the same as that of $Q$ (the body).

Assume $P$ and $Q$ are pointers into context transitions, and $c$ is a context. Then the lexical entry for the indefinite determiner $a$ looks like this:

$$
\lambda P \lambda Q \mapsto\left(u_{i} ; P i ; Q i\right)
$$


Abbreviating $\mathbb{N} \rightarrow[e] \rightarrow[e] \rightarrow t$ as $K$, we can express the type of this entry as $K \rightarrow K \rightarrow[e] \rightarrow[e] \rightarrow t$. An indefinite determiner translates into a function that takes a pointer into a context transition for the restriction, a pointer into a context transition for the body, and then yields a context transition.

What the entry for the indefinite determiner says is that the input context $c$ has length $i$, that it can be extended with a new element, and that this new element will satisfy both $P$ and $Q$, and it will remain accessible for future reference.

The final thing that is missing is the lift from unary predicates (the type of the regular denotation for entries like man) to pointers into context transitions. Here is a function for achieving that lift:

$$
\lambda A \lambda i \lambda c \lambda c^{\prime} \mapsto c=c^{\prime} \wedge A\left(c_{i}\right) .
$$

This takes a unary predicate ( $A$ has type $e \rightarrow t$ ) and yields a function of type $K$. The new function puts a constraint on the current context $c$, namely the constraint that the item at position $i$ in that context has to satisfy predicate $A$.

For the treatment of universal noun phrases like every man we need either a combination of dynamic negation and sequential composition, or an operation for dynamic implication. The way to express context negation or dynamic negation in DRT is this:

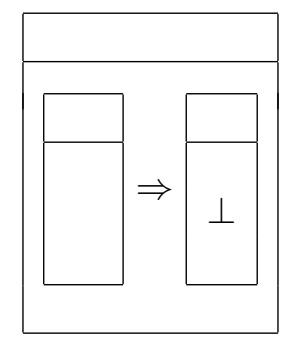

The typed logical version is the following, where $\phi$ represents the embedded representation structure.

$$
\neg \phi \quad:=\lambda c c^{\prime} \mapsto\left(c=c^{\prime} \wedge \neg \exists c^{\prime \prime} \phi c c^{\prime \prime}\right)
$$

This defines a relation between input context $c$ and output context $c^{\prime}$ where the input context equals the output context, and where there is no extension 
$c^{\prime \prime}$ of context $c$ for which $\phi$ holds. Abstracting from the embedded context transition, we get the following type logical definition for dynamic negation:

$$
\lambda \phi c c^{\prime} \mapsto\left(c=c^{\prime} \wedge \neg \exists c^{\prime \prime} \phi c c^{\prime \prime}\right)
$$

Dynamic implication can be defined in a similar way:

$$
\phi \Rightarrow \psi \quad:=\lambda c \lambda c^{\prime} \mapsto\left(c=c^{\prime} \wedge \forall c_{2}\left(\phi c c_{2} \rightarrow \exists c_{3} \psi c_{2} c_{3}\right) .\right.
$$

Abstracting from the two context transitions $\phi, \psi$, this gives:

$$
\lambda \phi \lambda \psi \lambda c \lambda c^{\prime} \mapsto\left(c=c^{\prime} \wedge \forall c_{2}\left(\phi c c_{2} \rightarrow \exists c_{3} \psi c_{2} c_{3}\right) .\right.
$$

Compare this to the truth definition for DRS conditions of the form

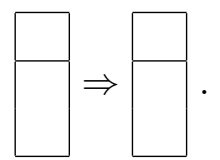

Now the lexical entry for the determiner every can be phrased in terms of dynamic implication, as follows:

$$
\lambda P \lambda Q \mapsto\left(\left(u_{i} ; P i\right) \Rightarrow Q i\right)
$$

Note that $\left(u_{i} ; P i\right) \Rightarrow Q i$ is a context transition, with type $[e] \rightarrow[e] \rightarrow t$. Therefore, the type of the translation of every is $K \rightarrow K \rightarrow[e] \rightarrow[e] \rightarrow t$.

Another way to phrase the lexical entry for the determiner every is as follows:

$$
\lambda P \lambda Q \lambda c \mapsto\left(\neg\left(u_{i} ; P i ; \neg Q i\right)\right) .
$$

It is left to the reader to check that this is equivalent to the definition in (2).

With these ingredients we give a compositional treatment of the following discourse:

$$
\text { A woman entered and a woman left. }
$$

Here are the representations for a woman, entered, and left:

$$
\begin{aligned}
a \text { woman: } & \lambda Q \lambda c \lambda c^{\prime} \mapsto \exists x\left(W x \wedge Q i \left(\hat{\left.\left.c^{\wedge} x\right) c^{\prime}\right)}\right.\right. & & \text { where } i=|c| \\
\text { entered: } & \lambda j \lambda c \lambda c^{\prime} \mapsto\left(c=c^{\prime} \wedge E c_{j}\right) & & \text { where } j \in|c| \\
\text { left: } & \lambda j \lambda c \lambda c^{\prime} \mapsto\left(c=c^{\prime} \wedge L c_{j}\right) & & \text { where } j \in|c|
\end{aligned}
$$


The notation $j \in|c|$ is shorthand for $j \in\{0, \ldots,|c|-1\}$. Note that the constraint on $j$ is in fact a restriction on the type of $j$ to a subset of $\mathbb{N}$.

The above entries yield the following representation for $A$ woman entered:

$$
\lambda c \lambda c^{\prime} \mapsto \exists x\left(W x \wedge E(\hat{c} x)[i] \wedge \hat{c} x=c^{\prime}\right)
$$

This reduces to (5).

$$
\lambda c \lambda c^{\prime} \mapsto \exists x\left(W x \wedge E x \wedge c^{\wedge} x=c^{\prime}\right)
$$

Similarly, we get the following as representation for $A$ woman left:

$$
\lambda c \lambda c^{\prime} \mapsto \exists x\left(W x \wedge L x \wedge \hat{c} x=c^{\prime}\right)
$$

Combining (5) and (6) by means of and (translated as $\lambda \phi \lambda \psi \mapsto \phi ; \psi)$ gives the following representation for $A$ woman entered and a woman left:

$$
\lambda c \lambda c^{\prime} \mapsto \exists x\left(W x \wedge E x \wedge \hat{c} x=c^{\prime}\right) ; \lambda c \lambda c^{\prime} \mapsto \exists x\left(W x \wedge L x \wedge \hat{c} x=c^{\prime}\right)
$$

This reduces to the following (note the renaming of variables):

$$
\lambda c \lambda c_{1} \mapsto \exists x\left(W x \wedge E x \wedge \hat{c} x=c_{1}\right) ; \lambda c_{2} \lambda c^{\prime} \mapsto \exists y\left(W y \wedge L y \wedge c_{2} \hat{y}=c^{\prime}\right)
$$

Applying the ; combinator and using $\beta$ reduction gives:

$$
\lambda c c^{\prime} \mapsto \exists c^{\prime \prime} \exists x\left(M x \wedge E x \wedge \hat{c^{\wedge} x}=c^{\prime \prime} \wedge \exists y\left(M y \wedge L y \wedge c^{\prime \prime \wedge} y=c^{\prime}\right)\right) .
$$

By simple equality reasoning, this reduces to:

$$
\lambda c \lambda c^{\prime} \mapsto \exists x\left(W x \wedge E x \wedge \exists y\left(W y \wedge L y \wedge \hat{c} x \wedge=c^{\prime}\right)\right) .
$$

So the interpretation of (3) sets up an appropriate context with references to two women, with the right constraints.

\section{B Pronouns and Anaphoric Reference}

The correct interpretation of pronouns and anaphoric definite descriptions should allow picking up appropriate references from the existing context.

A new customer entered the shop. He asked for the manager.

A new customer entered the shop. The man smiled. 
On the most salient reading of (7), he is interpreted as an anaphoric reference to a new customer, and the manager is interpreted as the manager of the shop. On the most salient reading of (8), the man is interpreted as an anaphoric reference to a new customer. To pick up such references, the available context information should include gender and number, actor focus (agent of the sentence), and discourse focus ('what is talked about' in the sentence). The most important, however, is the list of available referents.

Spelling out a full fledged anaphoric reference resolution mechanism is beyond the scope of this addendum. Instead, we will describe how anaphoric linking to a given object $c_{i}$ (or $c[i]$ in array notation) in context $c$ is to be implemented. So we assume that resolution in context $c$ has taken place, and we show that the linking to the appropriate item gets encoded by means of an appropriate lexical item for pronouns. More in particular:

$$
\text { A new customer entered the shop. He smiled. }
$$

Here, the index indicates that he gets resolved to the first introduced referent in context, i.e., to the referent for a new customer.

The following lexical entry for indexed pronouns accomplishes that the pronoun $h e_{i}$ gets resolved to item $i$ in context. Assume that $Q$ has type $e \rightarrow[e] \rightarrow[e] \rightarrow t$ and that $c, c^{\prime}$ have type $[e]$. Then the following translation for $h e_{i}$ has type $[e] \rightarrow[e] \rightarrow t$.

$$
h e_{i}: \quad \lambda Q \lambda c \lambda c^{\prime} \mapsto Q\left(c_{i}\right) c c^{\prime}
$$

\section{Once More: DRSs for Natural Language Frag- ments}

We can now redo the fragment from Section 10. If we start out with basic types $e$ for entities and $t$ for truth values, we can define the type of contexts as $[e]$ (lists of entities), that of context transitions $T$ as $[e] \rightarrow[e] \rightarrow t$, and that of indices into context transitions $K$ as $\mathbb{N} \rightarrow T$.

In the present set-up (unlike in that of Asher [4], Bos et al. [12], Kuschert [47], Muskens [50], and Section 10, there is no need to distinguish between variables of the typed logic and reference markers.

The composition of representation structures for example sentences from this fragment is a matter of routine. See Gamut [30] for a didactic account of the 


\begin{tabular}{|c|c|c|c|}
\hline expression & category & translates to & type \\
\hline $\mathrm{a}^{i}$ & $\operatorname{DET}(\mathrm{i}, *)$ & $\lambda P \lambda Q \lambda c \mapsto\left(u_{i} ; P i ; Q i\right)$ & $K \rightarrow K \rightarrow T$ \\
\hline every $^{i}$ & $\operatorname{DET}\left(\mathrm{i},{ }^{*}\right)$ & $\lambda P \lambda Q \lambda c \mapsto\left(u_{i} ; P i\right) \Rightarrow Q i$ & $K \rightarrow K \rightarrow T$ \\
\hline no $^{i}$ & $\operatorname{DET}\left(\mathrm{i},{ }^{*}\right)$ & $\lambda P \lambda Q \lambda c \mapsto \neg\left(u_{i} ; P i ; Q i\right)$ & $K \rightarrow K \rightarrow T$ \\
\hline $\operatorname{another}_{j}^{i}$ & $\operatorname{DET}(\mathrm{i}, \mathrm{j})$ & $\begin{array}{l}\lambda P \lambda Q \lambda c \mapsto u_{i} ; N E Q i j ; P i ; Q i \\
\text { where } N E Q \text { ij equals } \lambda c \lambda c^{\prime} \mapsto c=c^{\prime} \wedge c_{i} \neq c_{j}\end{array}$ & $\begin{array}{l}K \rightarrow K \rightarrow T \\
\mathrm{~T}\end{array}$ \\
\hline the $e_{j}^{i}$ & $\operatorname{DET}(\mathrm{i}, \mathrm{j})$ & $\begin{array}{l}\lambda P \lambda Q \lambda c \mapsto u_{i} ; E Q i j ; P i ; Q i \\
\text { where } E Q i j \text { equals } \lambda c \lambda c^{\prime} \mapsto c=c^{\prime} \wedge c_{i}=c_{j}\end{array}$ & $\begin{array}{l}K \rightarrow K \rightarrow T \\
\mathrm{~T}\end{array}$ \\
\hline $\operatorname{his}_{j}^{i}$ & $\operatorname{DET}(\mathrm{i}, \mathrm{j})$ & $\begin{array}{l}\lambda P \lambda Q \lambda c \mapsto u_{i} ; \text { POSS ji;Pi;Qi} \\
\text { where } P O S S j i \text { equals } \lambda c \lambda c^{\prime} \mapsto c=c^{\prime} \wedge \text { poss }\left(c_{j}, c_{i}\right)\end{array}$ & $\begin{array}{l}K \rightarrow K \rightarrow T \\
\mathrm{~T}\end{array}$ \\
\hline $\mathrm{Bill}_{i}$ & $\mathrm{NP}(*, *, i)$ & $\begin{array}{l}\lambda P \mapsto(I b i ; P i) \\
\text { where } I b i \text { equals } \lambda c \lambda c^{\prime} \mapsto c=c^{\prime} \wedge c_{i}=b\end{array}$ & $\begin{array}{l}K \rightarrow T \\
\mathrm{~T}\end{array}$ \\
\hline who & REL & $\lambda P \lambda Q \lambda i \mapsto(Q i ; P i)$ & $K \rightarrow K \rightarrow K$ \\
\hline he $_{i}$ & $\mathrm{NP}\left(\right.$ nom, $\left.{ }^{*}, \mathrm{i}\right)$ & $\lambda P \mapsto P i$ & $K \rightarrow T$ \\
\hline $\operatorname{him}_{i}$ & $\mathrm{NP}\left(\operatorname{acc},{ }^{*}, \mathrm{i}\right)$ & $\lambda P \mapsto P i$ & $K \rightarrow T$ \\
\hline $\operatorname{man}$ & $\mathrm{CN}$ & $\lambda i \lambda c \lambda c^{\prime} \mapsto\left(c=c^{\prime} \wedge \operatorname{man} c_{i}\right)$ & $K$ \\
\hline boy & $\mathrm{CN}$ & $\lambda i \lambda c \lambda c^{\prime} \mapsto\left(c=c^{\prime} \wedge\right.$ boy $\left.c_{i}\right)$ & $K$ \\
\hline smiles & VP(Tensed $)$ & $\lambda i \lambda c \lambda c^{\prime} \mapsto\left(c=c^{\prime} \wedge\right.$ smile $\left.c_{i}\right)$ & $K$ \\
\hline smile & $\mathrm{VP}(\operatorname{Inf})$ & $\lambda i \lambda c \lambda c^{\prime} \mapsto\left(c=c^{\prime} \wedge\right.$ smile $\left.c_{i}\right)$ & $K$ \\
\hline has & TV(Tensed) & $\begin{array}{l}\lambda \mathcal{P} \lambda i \mapsto(\mathcal{P}(\lambda j \mapsto(P O S S i j)) \\
\text { where } P O S S i j=\lambda c \lambda c^{\prime} \mapsto c=c^{\prime} \wedge \text { poss }\left(c_{i}, c_{j}\right)\end{array}$ & $\begin{array}{l}(K \rightarrow T) \rightarrow K \\
\mathrm{~T}\end{array}$ \\
\hline have & TV(Inf) & $\begin{array}{l}\lambda \mathcal{P} \lambda i \mapsto(\mathcal{P}(\lambda j \mapsto(P O S S i j)) \\
\text { where } P O S S i j=\lambda c \lambda c^{\prime} \mapsto c=c^{\prime} \wedge \text { poss }\left(c_{i}, c_{j}\right)\end{array}$ & $\begin{array}{l}(K \rightarrow T) \rightarrow K \\
\mathrm{~T}\end{array}$ \\
\hline hates & TV(Tensed) & $\lambda \mathcal{P} \lambda i \mapsto\left(\mathcal{P}\left(\lambda j \lambda c \lambda c^{\prime} \mapsto\left(c=c^{\prime} \wedge\right.\right.\right.$ hate $\left.\left.\left(c_{i}, c_{j}\right)\right)\right)$ & $(K \rightarrow T) \rightarrow K$ \\
\hline hate & TV(Inf) & $\lambda \mathcal{P} \lambda i \mapsto\left(\mathcal{P}\left(\lambda j \lambda c \lambda c^{\prime} \mapsto\left(c=c^{\prime} \wedge\right.\right.\right.$ hate $\left.\left.\left(c_{i}, c_{j}\right)\right)\right)$ & $(K \rightarrow T) \rightarrow K$ \\
\hline does not & AUX & $\lambda P \lambda i \mapsto \neg P i$ & $K \rightarrow K$ \\
\hline if & $(\mathrm{S} / \mathrm{S}) / \mathrm{S}$ & $\lambda p \lambda q \mapsto(p \Rightarrow q)$ & $T \rightarrow T \rightarrow T$ \\
\hline$\cdot$ & $\mathrm{S} \backslash(\mathrm{TXT} / \mathrm{S})$ & $\lambda p \lambda q \mapsto(p ; q)$ & $T \rightarrow T \rightarrow T$ \\
\hline . & $\mathrm{TXT} \backslash(\mathrm{TXT} / \mathrm{S})$ & $\lambda p \lambda q \mapsto(p ; q)$ & $T \rightarrow T \rightarrow T$ \\
\hline
\end{tabular}

Figure 11: Lexical component of the toy fragment for English. 
general procedure, and Van Eijck and Unger [27] for a textbook treatment including an implementation of dynamic semantics using the technique.

As in the previous fragment, there is an implicit assumption that the texts to which our theory is applied come fully equipped with all the necessary upper and lower indices and that all of these have been assigned in advance. Again, this assumption gets us into difficulties. Consider text (99).

(99) $\mathrm{A} \mathrm{man}^{i}$ who mistrusted the $\operatorname{assistant}_{k}^{j}$ walked in. $\mathrm{He}_{i}$ asked for the manager ${ }_{m}^{j}$. $\mathrm{He}_{j}$ turned out to be on holiday.

If this text is processed incrementally, first representations are built for the first two sentences and these are combined. Only then is a representation for the third sentence combined with the representation of the preceding discourse. Note that in this case the representation of the second sentence puts a constraint on the context produced by the first sentence that cannot be fulfilled. The input context contains a representation for the assistant, at position $j$, so it has length $>j$. The indexing for the manager would force the context to have length $j$, which is impossible. The solution is to choose a different upper index for the manager, to avoid the clash.

\section{Salience Updating as Context Manipulation}

A suitable interface for anaphora resolution needs to incorporate a notion of salience, or measure of availability of referents as candidates for resolution. Surface syntactic form is an important determinant for salience. E.g., it is usually assumed that a subject is more salient than an object. Thus, the first choice for resolving he in (10) is a farmer.

$$
\text { A farmer hit a gentleman. He was upset. }
$$

And the first choice for resolving he in (11) is a gentleman.

$$
\text { A gentleman was hit by a farmer. He was upset. }
$$

In these two examples, a farmer and a gentleman are the two obvious candidates for resolving the reference of the pronoun he, because both a farmer and a gentleman have been made salient by the preceding text. Consider the following context: 


\begin{tabular}{|l|l|l|}
\hline Pedro & Bernardo & Don Diego \\
\hline
\end{tabular}

Salience update in context is a reshuffle of the order of importance of the items in a context list. This may make Don Diego the most salient item:

\begin{tabular}{|l|l|l|}
\hline Don Diego & Pedro & Bernardo \\
\hline
\end{tabular}

To allow reshuffling of a context with Don Diego in it, in such a way that we do not lose track of him, we represent contexts as lists of indexed objects, with the indices running from 0 to the length of the context minus 1 :

\begin{tabular}{|c|c|c|}
\hline \multicolumn{1}{|c}{1} & 2 \\
\hline Don Diego & Bernardo & Pedro \\
\hline
\end{tabular}

Reshuffling this to make Pedro most salient gives:

\begin{tabular}{|c|c|c|}
\hline 2 & 0 & 1 \\
\hline Pedro & Don Diego & Bernardo \\
\hline
\end{tabular}

Note that the indices $0, \ldots, n-1$ determine a permutation of the context list. We call these lists of indexed objects contexts under permutation.

In a context $c$, the entity with index $i$ is given by $c[* i]$.

$$
\left(\begin{array}{c|c|c}
2 & 0 & 1 \\
\hline \text { Pedro } & \text { Don Diego } & \text { Bernardo } \\
\hline
\end{array}\right)[* 0]=\text { Don Diego }
$$

If $c$ is a context under permutation, let $(i) c$ be the result of placing the item $(i, c[* i])$ upfront. Here is an example:

(1)

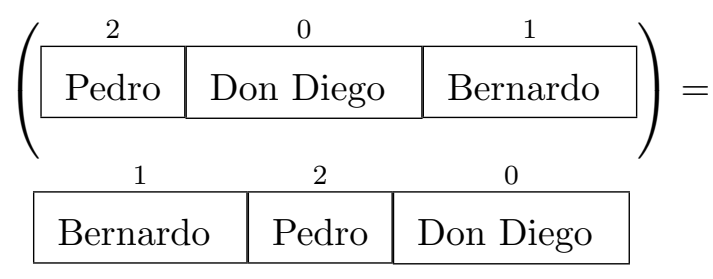


(i) $c$ is the result of moving the item with index $i$ to the head position of the context list. Successive applications of this operation can generate all permutations of a context. If $d$ is an object and $c$ a context, then $d: c$ is the result of putting item $(|c|, d)$ at the head position of the context list.

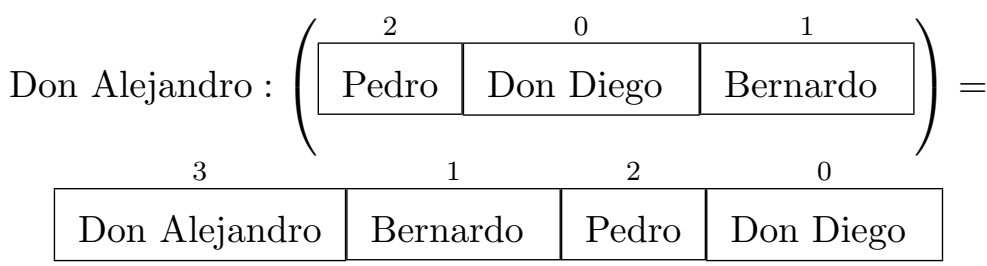

The operation (:) is used for adding a new element to the context, in most salient position. Using this, and introducing a type $p[e]$ for contexts under permutation, we can give a type theoretical version of discourse representation that allows salience updating. Assume $c, c^{\prime}$ are variables of type $p[e]$, and $P, Q$ are variables of type $\mathbb{N} \rightarrow p[e] \rightarrow p[e] \rightarrow t$. Then the new definition of context extension runs as follows:

$$
\exists:=\lambda c c^{\prime} \mapsto \exists x\left((x: c)=c^{\prime}\right)
$$

Here $(x: c)$ means that $x$ is added to the context, at the most salient position. The lift of unary predicates to pointers into context-under-permutation transitions that is necessary to make this work is defined as:

$$
\lambda A \lambda i \lambda c \lambda c^{\prime} \mapsto c=c^{\prime} \wedge A(c[* i]) .
$$

The new translation of a man effects a salience reshuffle:

$$
\lambda Q c c^{\prime} \mapsto|c|=i \wedge \exists x\left(\operatorname{Man} x \wedge Q i(x: c) c^{\prime}\right) .
$$

The referent $x$ for the indefinite gets put in most salient position in the new context by means of the operation $x: c$. Note that $(x: c)[* i]$ will pick up $x$.

\section{E Further Reading}

Two key publications on discourse representation are Heim [35] and Kamp [37], which address themselves specifically to the problem of the interpretation of indefinite descriptions and their interaction with unbound and transsentential anaphora. Temporal anaphora, a kind of anaphora that is 
largely transsentential, is treated along the same lines in Kamp and Rohrer [41]. A systematic presentation of discourse representation theory including various later developments is given in Kamp and Reyle [38]. Asher [4] extends DRT to a more comprehensive theory which among other things also takes discourse structure and rhetorical relations into account. The connections between the principles of DRT and those of generative syntax are explored in depth in Chierchia [14]. Questions of lexical semantics from a DR-theoretical perspective are explored in Kamp and Rossdeutscher [42].

A precursor paper is Karttunen [44]. Examples of related approaches to semantics which have also advocated focusing on the discourse level are Seuren's discourse semantics [62], Barwise's dynamic interpretation of anaphora [7], and the game theoretical school of Hintikka c.s. [36].

Further references on the connection with dynamic reasoning are given in the Chapter on Dynamics in this Handbook. Connections between discourse representation and type theory are sketched in Ahn and Kolb [1]. Connections between discourse representation and game theoretical semantics are given in Van Benthem and Van Eijck [9].

The mathematics of context and context extension has developed into a topic in its own right; see, e.g., [71]. A version of DRT called incremental dynamics is presented in [23]. This framework can be viewed as the one-variable version of sequence semantics for dynamic predicate logic, as proposed in [67]. Incremental dynamics is described in terms of polymorphic type theory in [22]. This system makes clear how the instruction to take fresh discourse referents when needed can be made fully precise by using the standard toolset of (polymorphic) type theory. Such a reconstruction of DRT in type theory does justice to the incrementality and the finite state semantics of the original. The proposal for the treatment of salience in Section $\mathrm{D}$ of this addendum is worked out as a fragment with a mechanism for reference resolution in a textbook chapter in [27]. This treatment should be compared with the treatment of pronoun resolution in DRT proposed in the second volume of [11], as well as with the earlier proposal for pronoun resolution in DRT in [72]. Discourse semantics in the style of Kamp and Heim can be viewed as a form of continuation passing style semantics for texts. This connection is worked out further in [17] and [6].

To change a function $f$ of type $a \rightarrow b$ into a continuation passing style function $f^{\prime}$ of type $a \rightarrow(b \rightarrow c) \rightarrow c$, one can define $f^{\prime}$ as $\lambda x \lambda g \mapsto g(f x)$. Then $f^{\prime}$ is a function that first takes an $x$, next takes a continuation function 
$g$, then starts to computes like $f$, but instead of returning the result $f x$, applies the function $g$ to that result.

If we look at this more closely, we see that this can be viewed as a combination of application and argument raising, as follows. Define $R(x)$ as $\lambda g \mapsto g x$. Then $f^{\prime}$ can be redefined as $\lambda x \mapsto R(f x)$. The reader who is familiar with Montague semantics has no doubt seen this argument raising before. This is how Montague grammar deals with proper names, to assimilate them to the type of generalized quantifiers. Reynolds, in his overview of the use of continuations in computer science [57], shows that continuations were invented over and over again. We can add that the person who reinvented them first for natural language semantics was Richard Montague.

In the case of discourse representation another kind of lifting takes place. Instead of just interpreting a piece of discourse in context, a second context is returned that can be interpreted in context next, according to the recipe:

$$
\phi c=\lambda c^{\prime} \mapsto \phi c c^{\prime} .
$$

To define truth, one has to step out of the continuation, by means of:

$$
T(\phi c)=\exists c^{\prime}\left(\phi c c^{\prime}\right) .
$$

Truth is a derived notion. To say that a text is true in context $c$ boils down to the statement that the context $c$ can be extended to $c^{\prime}$, all relative to some model $M$ in which $c$ is embedded.

Besides the connections with type theory and continuations, there is a link with dynamic logic and Hoare style correctness reasoning in programming. DPL, a close cousin of DRT, can be viewed as a fragment of quantified dynamic logic. For the tracing of this connection we refer to [25]. For a still broader perspective on natural language text processing as information processing we refer to [43], in particular Chapter 3, 'Meaning in Context'.

Discourse representation theory has found its way into implementations of large scale natural language processing systems. A prominent example of this is the aptly named Boxer system for building and manipulating DRT box representations of natural language texts. See [15].

Speaking very generally, discourse is an act of communication that establishes common knowledge between a speaker and an audience. As the discourse processes, speaker and audience may switch roles. Beliefs are updated in the course of communication, but the discourse situation also employs 
common knowledge and common belief (what the speaker knows or believes about the audience) to establish communication. The update idea that announcing a proposition $\phi$ removes all worlds where $\phi$ does not hold is old logical folklore; explicit statements can be found since the 1970s in the works of Stalnaker, Heim, and others. The same idea also served as a high-light in the work on epistemic logic in computer science (cf. [28]). Its first implementation as a dynamic-epistemic logic is due to Plaza [53] (see also [32]). The public announcement update idea was generalized in [5]; a streamlined version of a general logic of communication and change is in [10], and a textbook treatment is in [18]. In the last chapter of [27] a textbook treatment of presupposition and question answering within this framework is given.

The study of social mechanisms may offer an extended agenda for natural language analysis, with the analysis of natural language communication in settings where something more definite than just information exchange is the focus: achievement of some well stated goals given by specific social protocols. See [51] and [26].

Acknowledgements Thanks to Johan van Benthem and Valeria de Paiva for support, hints and suggestions.

\section{References}

[1] R. Ahn and H.-P. Kolb. Discourse representation meets constructive mathematics. In L. Kalman and L. Polos, editors, Papers from the Second Symposium on Logic and Language, pages 105-124. Akademiai Kiadoo, Budapest, 1990.

[2] H. Alshawi, editor. The Core Language Engine. MIT Press, Cambridge Mass, Cambridge, Mass., and London, England, 1992.

[3] N. Asher. Belief in discourse representation theory. Journal of Philosophical Logic, 15:127-189, 1986.

[4] N. Asher. Reference to Abstract Objects in Discourse. Kluwer, Dordrecht, Dordrecht, 1993.

[5] A. Baltag, L. S. Moss, and S. Solecki. The logic of public announcements, common knowledge, and private suspicions. Technical Report SEN-R9922, CWI, Amsterdam, 1999. Many updates. 
[6] Chris Barker and Chung-chieh Shan. Donkey anaphora is in-scope binding. Semantics and Pragmatics, 1(1):1-46, 2008.

[7] J. Barwise. Noun phrases, generalized quantifiers and anaphora. In P. Gärdenfors, editor, Generalized Quantifiers: linguistic and logical approaches, pages 1-30. Reidel, Dordrecht, 1987.

[8] J. van Benthem. The Logic of Time. Reidel, Dordrecht, Dordrecht, 1983.

[9] J. van Benthem and J. van Eijck. The dynamics of interpretation. Journal of Semantics, 1(1):3-20, 1982.

[10] J. van Benthem, J. van Eijck, and B. Kooi. Logics of communication and change. Information and Computation, 204(11):1620-1662, 2006.

[11] P. Blackburn and J. Bos. Representation and Inference for Natural Language; A First Course in Computational Semantics. CSLI Lecture Notes, 2005.

[12] J. Bos, E. Mastenbroek, S. McGlashan, S. Millies, and M. Pinkal. A compositional DRS-based formalism for NLP-applications. In Proceedings of the International Workshop on Computational Linguistics, pages 21-31. University of Tilburg, 1994.

[13] O. Bouchez, J. van Eijck, and O. Istace. A strategy for dynamic interpretation: a fragment and an implementation. In S. Krauwer, M. Moortgat, and Louis des Tombe, editors, Sixth Conference of the European Chapter of the Association for Computational Linguistics Proceedings of the Conference, pages 61-70. ACL, 1993.

[14] G. Chierchia. The Dynamics of Meaning. The University of Chicago Press, Chicago and London, 1995.

[15] James R. Curran, Stephen Clark, and Johan Bos. Linguistically motivated large-scale nlp with c\&c and boxer. In $A C L$ '0\%: Proceedings of the 45th Annual Meeting of the ACL on Interactive Poster and Demonstra tion Sessions, pages 33-36, Morristown, NJ, USA, 2007. Association for Computational Linguistics.

[16] D. Davidson. The logical form of action sentences. In N. Rescher, editor, The Logic of Decision and Action, pages 81-95. The University Press, Pittsburgh, 1967. 
[17] Philippe de Groote. Towards a Montegovian account of dynamics. In Proceedings of Semantics and Linguistic Theory XVI, CLC Publications, 2004.

[18] H.P. van Ditmarsch, W. van der Hoek, and B. Kooi. Dynamic Epistemic Logic, volume 337 of Synthese Library. Springer, 2006.

[19] J. van Eijck. Merge reduction in dynamic semantics. Manuscript, CWI, Spring1996.

[20] J. van Eijck. The dynamics of description. Journal of Semantics, 10:239-267, 1993.

[21] J. van Eijck. Axiomatizing dynamic predicate logic with quantified dynamic logic. In J. van Eijck and A. Visser, editors, Logic and Information Flow, pages 30-48. MIT Press, Cambridge Mass, 1994.

[22] J. van Eijck. The proper treatment of context in NL. In Paola Monachesi, editor, Computational Linguistics in the Netherlands 1999; Selected Papers from the Tenth CLIN Meeting, pages 41-51. Utrecht Institute of Linguistics OTS, 2000.

[23] J. van Eijck. Incremental dynamics. Journal of Logic, Language and Information, 10:319-351, 2001.

[24] J. van Eijck and H. Kamp. Representing discourse in context. In J. van Benthem and A. ter Meulen, editors, Handbook of Logic and Language, pages 179-237. Elsevier, Amsterdam, 1997.

[25] J. van Eijck and M. Stokhof. The gamut of dymamic logics. In D.M. Gabbay and J. Woods, editors, The Handbook of the History of Logic, volume 7 - Logic and the Modalities in the Twentieth Century, pages 499-600. Elsevier, 2006.

[26] J. van Eijck and R. Verbrugge, editors. Discourses on Social Software, volume 5 of Texts in Logic and Games. Amsterdam University Press, Amsterdam, 2009.

[27] Jan van Eijck and Christina Unger. Computational Semantics with Functional Programming. To appear with Cambridge University Press, 2009 .

[28] R. Fagin, J.Y. Halpern, Y. Moses, and M.Y. Vardi. Reasoning About Knowledge. MIT Press, Cambridge, Massachusetts, 1995. 
[29] G. Frege. Ueber sinn und bedeutung. Translated as 'On Sense and Reference' in Geach and Black (eds.), Translations from the Philosophical Writings of Gottlob Frege, Blackwell, Oxford (1952), 1892.

[30] L.T.F. Gamut. Language, Logic and Meaning, Part 2. Chicago University Press, Chicago, 1991.

[31] P.T. Geach. Reference and Generality: An Examination of Some Medieval and Modern Theories. Cornell University Press, Ithaca, 1962 (Third revised edition: 1980).

[32] J. Gerbrandy. Bisimulations on Planet Kripke. ILLC Dissertation Series, Amsterdam, 1999.

[33] J. Groenendijk and M. Stokhof. Dynamic predicate logic. Linguistics and Philosophy, 14:39-100, 1991.

[34] M.A.K. Halliday and R. Hassan. Cohesion in English. Longman, London, 1976.

[35] I. Heim. The Semantics of Definite and Indefinite Noun Phrases. PhD thesis, University of Massachusetts, Amherst, 1982.

[36] J. Hintikka and J. Kulas. Anaphora and Definite Descriptions: Two Applications of Game-Theoretical Semantics. Reidel, Dordrecht, 1985.

[37] H. Kamp. A theory of truth and semantic representation. In J. Groenendijk, T. Janssen, and M. Stokhof, editors, Formal Methods in the Study of Language, pages 277-322. Mathematisch Centrum, Amsterdam, 1981.

[38] H. Kamp and U. Reyle. From Discourse to Logic. Kluwer, Dordrecht, 1993.

[39] H. Kamp and U. Reyle. A calculus for first order discourse representation structures. Journal of Logic, Language and Information, 5(34):297-348, 1996.

[40] H. Kamp and U. Reyle. Discourse representation theory. In Maienborn, Portner, and Von Heusinger, editors, Handbuch Semantik. Walter de Gruyter, to appear.

[41] H. Kamp and C. Rohrer. Tense in texts. In Bäuerle, Schwarze, and Von Stechow, editors, Meaning, Use and Interpretation of Language, pages 250-269. De Gruyter, Berlin, 1983. 
[42] H. Kamp and A. Rossdeutscher. Remarks on lexical structure and DRS construction. Theoretical Linguistics, 20(2/3), 1994.

[43] Hans Kamp and Martin Stokhof. Information in natural language. In Johan van Benthem and Pieter Adriaans, editors, Philosophy of Information, volume 8 of Handbook of the Philosophy of Science, pages 49-111. North Holland, 2008.

[44] L. Karttunen. Discourse referents. In J. McCawley, editor, Syntax and Semantics 7, pages 363-385. Academic Press, 1976.

[45] J.W. Klop. Term rewriting systems. In S. Abramski, D. Gabbay, and T. Maibaum, editors, Handbook of Logic in Computer Science, pages 1-116. Oxford University Press, 1992.

[46] S.A. Kripke. Naming and necessity. In D. Davidson and G. Harman, editors, Semantics of Natural Language, pages 253-355. Reidel, Dordrecht, 1972.

[47] S. Kuschert. Eine Erweiterung des $\lambda$-Kaküls um Diskursrepresentationsstrukturen. Master's thesis, Universität des Saarlandes, 1995.

[48] W.C. Mann and S.A. Thompson. Rhetorical structure theory: A framework for the analysis of texts. IPRA Papers in Pragmatics, 1:1-21, 1987.

[49] R. Muskens. Tense and the logic of change. In U. Egli et al., editor, Lexical Knowledge in the Organization of Language, pages 147-183. W. Benjamins, 1995.

[50] R. Muskens. Combining Montague Semantics and Discourse Representation. Linguistics and Philosophy, 19:143-186, 1996.

[51] Rohit Parikh. Social software. Synthese, 132:187-211, 2002.

[52] B. Partee. Some structural analogies between tenses and pronouns in english. Journal of Philosophy, 70:601-9, 1973.

[53] J. A. Plaza. Logics of public communications. In M. L. Emrich, M. S. Pfeifer, M. Hadzikadic, and Z. W. Ras, editors, Proceedings of the 4 th International Symposium on Methodologies for Intelligent Systems, pages 201-216, 1989.

[54] H. Reichenbach. Elements of Symbolic Logic. Macmillan, London, 1947. 
[55] U. Reyle. Dealing with ambiguities by underspecification: Construction, representation and deduction. Journal of Semantics, 10:123-179, 1993.

[56] U. Reyle. On reasoning with ambiguities. In Seventh Conference of the European Chapter of the Association for Computational Linguistics Proceedings of the Conference, 1995.

[57] J.C. Reynolds. The discoveries of continuations. Lisp and Symbolic Computation, 6(3-4):233-247, 1993.

[58] B. Russell. On denoting. Mind, 14:479-493, 1905.

[59] R.A. van der Sandt. Presupposition projection as anaphora resolution. Journal of Semantics, 9:333-377, 1992. Special Issue: Presupposition, Part 2.

[60] W. Saurer. A natural deduction system of discourse representation theory. Journal of Philosophical Logic, 22(3):249-302, 1993.

[61] C. Sedogbo and M. Eytan. A tableau calculus for DRT. Logique et Analyse, 31:379-402, 1988.

[62] P. Seuren. Discourse Semantics. Blackwell, Oxford, 1986.

[63] C.L. Sidner. Towards a Computation Theory of Definite Anaphora Comprehension in English Discourse. PhD thesis, MIT, Cambridge, 1979.

[64] R. Stalnaker. Pragmatic presuppositions. In M.K. Munitz and P.K. Unger, editors, Semantics and Philosophy, pages 197-213. New York University Press, 1974.

[65] P.F. Strawson. On referring. Mind, 59:320-344, 1950.

[66] H. Verkuyl. On the Compositional Nature of the Aspects. PhD thesis, University of Utrecht, Utrecht, 1972.

[67] C.F.M. Vermeulen. Sequence semantics for dynamic predicate logic. Journal of Logic, Language, and Information, 2:217-254, 1993.

[68] C.F.M. Vermeulen. Merging without mystery. Journal of Philosophical Logic, 24:405-450, 1995. 
[69] A. Visser. Actions under presuppositions. In J. van Eijck and A. Visser, editors, Logic and Information Flow, pages 196-233. MIT Press, 1994.

[70] A. Visser. The design of dynamic discourse denotations. Lecture notes, Utrecht University, 1994.

[71] A. Visser and C. Vermeulen. Dynamic bracketing and discourse representation. Notre Dame Journal of Formal Logic, 37:321-365, 1996.

[72] H. Wada and N. Asher. BUILDRS: An implementation of DR theory and LFG. In 11th International Conference on Computational Linguistics. Proceedings of Coling '86, pages 540-545, University of Bonn, 1986.

[73] B. Webber. A Formal Approach to Discourse Anaphora. Garland, New York, 1979.

[74] H. Zeevat. A compositional approach to discourse representation theory. Linguistics and Philosophy, 12:95-131, 1989. 